\title{
Interval-Valued Pythagorean Hesitant Fuzzy Set and Its Application to Multiattribute Group Decision-Making
}

\author{
Maoyin Zhang, ${ }^{1}$ Tingting Zheng $\mathbb{D}^{1}{ }^{1}$ Wanrong Zheng, ${ }^{1}$ and Ligang Zhou ${ }^{1,2}$ \\ ${ }^{1}$ School of Mathematical Sciences, Anhui University, Hefei 230601, China \\ ${ }^{2}$ China Institute of Manufacturing Development, Nanjing University of Information Science and Technology, \\ Nanjing 210044, China \\ Correspondence should be addressed to Tingting Zheng; tt-zheng@163.com
}

Received 4 May 2019; Revised 22 July 2019; Accepted 12 August 2019; Published 13 February 2020

Academic Editor: Lingzhong Guo

Copyright (c) 2020 Maoyin Zhang et al. This is an open access article distributed under the Creative Commons Attribution License, which permits unrestricted use, distribution, and reproduction in any medium, provided the original work is properly cited.

\begin{abstract}
Pythagorean hesitant fuzzy sets are widely watched because of their excellent ability to deal with uncertainty, imprecise and vague information. This paper extends Pythagorean hesitant fuzzy environments to interval-valued Pythagorean hesitant fuzzy environments and proposes the concept of interval-valued Pythagorean hesitant fuzzy set (IVPHFS), which allows the membership of each object to be a set of several pairs of possible interval-valued Pythagorean fuzzy elements. Furthermore, we develop a series of aggregation operators for interval-valued Pythagorean hesitant fuzzy information and apply them to multiattribute group decision-making (MAGDM) problems. Then, some desired operational laws and properties of IVPHFSs are studied. Especially, considering an interval-valued Pythagorean fuzzy element (IVPHFE) is formed by several pairs of interval values, this paper proposes the concepts of score function and accuracy function in the form of two interval numbers which can retain intervalvalued Pythagorean fuzzy information as much as possible. Then, the relationship among these operators is discussed by comparing the interval numbers. Eventually, an illustrative example fully shows the feasibility, practicality, and effectiveness of the proposed approach.
\end{abstract}

\section{Introduction}

There are numerous uncertain, imprecise, and incomplete problems in the real world. Zadeh's fuzzy set theory [1] is a successful and effective tool to solve many similar problems. The theory and its primary extensions, such as intuitionistic fuzzy sets [2], interval-valued intuitionistic fuzzy sets [3], type-2 fuzzy sets [4], interval type-2 fuzzy sets [5], and $q$ rung orthopair fuzzy sets [6], have been successfully applied to pattern recognition [7], medical diagnosis [8], fuzzy logic [9], decision-making [10-18], and so on.

In many practical cases, it is difficult to define a membership function of a universe because people would face several possible membership degrees of one object to be chosen and hesitate about which one would be the most right one. Hesitant fuzzy set theory, introduced by Torra and Narukawa $[19,20]$, has provided successful results dealing with hesitant situations, which are not well managed by the previous tools [21]. It constructs possible membership degrees of an object as a set and keeps more information in real environment. Many scholars have focused on hesitant fuzzy sets and proposed diverse corresponding extensions, such as dual hesitant fuzzy sets [22], interval-valued dual fuzzy sets $[23,24]$, intuitionistic hesitant fuzzy sets [25], intervalvalued hesitant fuzzy sets [26-28], and interval-valued intuitionistic fuzzy sets [29]. These theories have been applied to decision-making [30,31], clustering analysis [32], and so on.

As another extension of fuzzy set theory, Yager $[33,34]$ proposed another class of nonstandard fuzzy sets, called Pythagorean fuzzy sets. The sets are represented by pairs of two values $\left\langle\mu_{P}(x), \nu_{P}(x)\right\rangle$, which satisfies $\mu_{P}^{2}(x)+\nu_{P}^{2}(x) \leq 1$. Obviously, their application range is broader than that of intuitionistic fuzzy sets. These situations are more common in different real-world problems. So, Pythagorean fuzzy sets have been paid attention in a short period of time. Yager 
[35, 36], Zhang and Xu [37], Ren et al. [38], Liu et al. [39], and Teng et al. [39] have studied several kinds of Pythagorean fuzzy aggregation operators and applied them to decision-making problems. Furthermore, Peng and Yang [40] proposed the definition of interval-valued Pythagorean fuzzy set and Rahamn et al. [41, 42] developed group decision-making with interval-valued Pythagorean fuzzy environments. Yi et al. [43] applied it to multicriteria decisionmaking problems. Also, Liang et al. [44] introduced the interval-valued Pythagorean fuzzy extended Bonferroni mean operators.

Recently, some scholars have tried to combine Pythagorean fuzzy sets and hesitant fuzzy sets, and all called them Pythagorean hesitant fuzzy sets (PHFSs), but their construction methods are controversial. Let $A=\left\{\left\langle x, \Gamma_{A}(x), \Psi_{A}(x)\right\rangle \mid x \in U\right\}$ be a Pythagorean fuzzy set in $U$, where $\Gamma_{A}(x)=\left\{\mu_{A}(x) \mid \mu_{A}(x) \in[0,1]\right\}$ and $\Psi_{A}(x)=\left\{v_{A}(x) \mid v_{A}(x) \in[0,1]\right\}$. Liu and He [45] defined that, for any $x$, any $\mu_{A}(x) \in \Gamma_{A}(x)$, and any $v_{A}(x) \in \Psi_{A}(x)$, $\left(\mu_{A}(x)\right)^{2}+\left(v_{A}(x)\right)^{2} \leq 1$ holds. Khan et al. [46] defined that, for any $x$ and for any $\mu_{A}(x) \in \Gamma_{A}(x)$, there is $\nu_{A}(x) \in \Psi_{A}(x)$, such that $\left(\mu_{A}(x)\right)^{2}+\left(\nu_{A}(x)\right)^{2} \leq 1$. Also, for any $v_{A}(x) \in \Psi_{A}(x)$, there is $\mu_{A}(x) \in \Gamma_{A}(x)$, such that $\left(\mu_{A}(x)\right)^{2}+\left(\nu_{A}(x)\right)^{2} \leq 1$. Wei et al.[47] also defined another Pythagorean fuzzy set $P=\left\{\left\langle x, h_{P}(x)\right\rangle \mid x \in U\right\}$, where $h_{P}(x)$ is a set of some Pythagorean fuzzy elements in $U$. The three definitions have their own merits. Considering the pairing of possible membership degrees and possible nonmembership degrees, it is more conducive to the aggregation of hesitant fuzzy numbers. In this paper, we choose Wei's definition as the definition of PHFS.

From the above analysis, we can see that PHFSs are more convenient to deal with fuzzy information than hesitant fuzzy sets or Pythagorean fuzzy sets. However, for real multiattribute group decision-making (MAGDM) problems, it is difficult for decision makers to provide some exact and crisp fuzzy values to depict uncertain or insufficient alternatives because of the increasing complexity of social and economic life. The aim of this paper is to extend PHFSs to interval-valued Pythagorean hesitant fuzzy sets (IVPHFSs) and develop MAGDM approaches to intervalvalued Pythagorean hesitant fuzzy environments based on newly constructed aggregation operators. In particular, since there is no one-to-one correspondence between interval numbers and real numbers, this paper directly uses interval numbers to define score functions and accuracy functions, which can preserve interval-valued Pythagorean fuzzy information as much as possible.

The rest of this paper is organized as follows. In Section 2, we review some basic concepts and results of hesitant fuzzy sets, interval-valued hesitant fuzzy sets, and intervalvalued Pythagorean fuzzy sets. Section 3 proposes the definition of IVPHFS and discusses some basic operational laws. In particular, we propose the concepts of the score function and accuracy function which are both appeared as interval values. Some operators for aggregating intervalvalued Pythagorean hesitant fuzzy information are studied and developed in section 4 . Section 5 shows the application to MAGDMs in interval-valued Pythagorean hesitant fuzzy environments and illustrates the feasibility and applicability of the proposed method. Concluding remarks are made in Section 6.

\section{Preliminaries}

This section will briefly review the basic notations and results of hesitant fuzzy sets, interval-valued Pythagorean fuzzy sets.

Hereinafter, without explanation, let $U$ be a nonempty finite set, called the universe of discourse, and $D[x, y]$ be the set of all closed subintervals of the interval $[x, y]$.

2.1. Hesitant Fuzzy Set (HFS). HFS was introduced by Torra and Narukawa [19] and Torra [20], which permits the membership being a set of possible values. It is very suitable for describing problems that are difficult to determine with only one membership.

Definition 1 (see [20]). A hesitant fuzzy set (HFS) $E$ on $U$ is described as $E=\left\{\left\langle x, h_{E}(x)\right\rangle \mid x \in U\right\}$, where $h_{E}(x)=$ $\left\{\mu_{E}(x) \mid \mu_{E}(x) \in[0,1]\right\}$ represents the set of possible membership degrees of $E$ at $x$.

For convenience, Xia and $\mathrm{Xu}$ [30] proposed the concept of a hesitant fuzzy element (HFE), denoted by $h=h_{E}(x)$, where $h=\{\gamma \mid \gamma \in[0,1]\}$. They gave some operations on HFEs.

Definition 2 (see $[19,20,30])$. Let $h, h_{1}$, and $h_{2}$ be three HFEs and $\lambda>0$, then

(1) $h^{C}=\{1-\gamma \mid \gamma \in h\}$

(2) $h^{\lambda}=\left\{\gamma^{\lambda} \mid \gamma \in h\right\}$

(3) $\lambda h=\left\{1-(1-\gamma)^{\lambda} \mid \gamma \in h\right\}$

(4) $h_{1} \oplus h_{2}=\left\{\gamma_{1}+\gamma_{2}-\gamma_{1} \gamma_{2} \mid \gamma_{1} \in h_{1}, \gamma_{2} \in h_{2}\right\}$

(5) $h_{1} \otimes h_{2}=\left\{\gamma_{1} \gamma_{2} \mid \gamma_{1} \in h_{1}, \gamma_{2} \in h_{2}\right\}$

(6) $h_{1} \cup h_{2}=\left\{\max \left\{\gamma_{1}, \gamma_{2}\right\} \mid \gamma_{1} \in h_{1}, \gamma_{2} \in h_{2}\right\}$

(7) $h_{1} \cap h_{2}=\left\{\min \left\{\gamma_{1}, \gamma_{2}\right\} \mid \gamma_{1} \in h_{1}, \gamma_{2} \in h_{2}\right\}$

Furthermore, to compare the HFEs, the following comparison laws are given.

Definition 3 (see [30]). For an $\operatorname{HFE} h, S(h)=1 /|h| \sum_{\gamma \in h} \gamma$ is called the score function of $h$ with $|\cdot|$ denoting the cardinality here and below. For two HFEs, $h_{1}$ and $h_{2}$, if $S\left(h_{1}\right)>S\left(h_{2}\right)$, then $h_{1}$ is superior to $h_{2}$, denoted by $h_{1}>h_{2}$; if $S\left(h_{1}\right)=S\left(h_{2}\right)$, then $h_{1}$ is equivalent to $h_{2}$, denoted by $h_{1}=h_{2}$.

2.2. Interval-Valued Hesitant Fuzzy Set (IVHFS). Chen et al. $[26,27]$ generalized HFSs to interval-valued hesitant fuzzy sets (IVPHFSs), in which the membership degree of each object of the universe is denoted by several possible interval values.

Definition 4 (see [26]). An interval-valued hesitant fuzzy set (IVHFS) $\widetilde{E}$ on $U$ is described as $\widetilde{E}=\left\{\left\langle x, \widetilde{h}_{\widetilde{E}}(x)\right\rangle \mid x \in U\right\}$, 
where $\widetilde{h}_{\widetilde{E}}(x)=\left\{\widetilde{\mu}_{\widetilde{E}}(x) \mid \widetilde{\mu}_{\widetilde{E}}(x) \triangleq\left[\mu_{\widetilde{E}}^{-}(x), \mu_{\widetilde{E}}^{+}(x)\right] \in D[0,1]\right\}$ represents the set of possible membership intervals of $\widetilde{E}$ at $x$.

For convenience, $\widetilde{h}_{\widetilde{E}}(x)$ is called an interval-valued hesitant fuzzy element (IVHFE), denoted by $\widetilde{h}=$ $\left\{\widetilde{\mu} \mid \widetilde{\mu} \triangleq\left[\mu^{-}, \mu^{+}\right]\right\}$. Especially, if $\mu^{-}=\mu^{+}$, then $\widetilde{h}$ degenerates to an HFS.

Definition 5 (see $[26,27])$. Let $\widetilde{h}, \widetilde{h}_{1}$, and $\widetilde{h}_{2}$ be three IVHFEs and $\lambda>0$, then

(1) $\tilde{h}^{C}=\left\{\left[1-\mu^{+}, 1-\mu^{-}\right] \mid \tilde{\mu} \in \tilde{h}\right\}$

(2) $\tilde{h}^{\lambda}=\left\{\left[\left(\mu^{-}\right)^{\lambda},\left(\mu^{+}\right)^{\lambda}\right] \mid \tilde{\mu} \in \widetilde{h}\right\}$

(3) $\lambda \widetilde{h}=\left\{\left[1-\left(1-\mu^{-}\right)^{\lambda}, 1-\left(1-\mu^{+}\right)^{\lambda}\right] \mid \widetilde{\mu} \in \widetilde{h}\right\}$

(4) $\widetilde{h}_{1} \oplus \widetilde{h}_{2}=\left\{\left[\mu_{1}^{-}+\mu_{2}^{-}-\mu_{1}^{-} \cdot \mu_{2}^{-}, \mu_{1}^{+}+\mu_{2}^{+}-\mu_{1}^{+} \cdot \mu_{2}^{+}\right]\right.$ $\left.\mid \widetilde{\mu}_{1} \in \widetilde{h}_{1}, \widetilde{\mu}_{2} \in \widetilde{h}_{2}\right\}$

(5) $\widetilde{h}_{1} \otimes \widetilde{h}_{2}=\left\{\left[\mu_{1}^{-} \cdot \mu_{2}^{-}, \mu_{1}^{+} \cdot \mu_{2}^{+}\right] \mid \widetilde{\mu}_{1} \in \widetilde{h}_{1}, \widetilde{\mu}_{2} \in \widetilde{h}_{2}\right\}$

(6) $\widetilde{h}_{1} \cup \widetilde{h}_{2}=\left\{\left[\max \left\{\mu_{1}^{-}, \mu_{2}^{-}\right\}, \max \left\{\mu_{1}^{+}, \mu_{2}^{+}\right\}\right]\right.$ $\left.\mid \widetilde{\mu}_{1} \in \widetilde{h}_{1}, \widetilde{\mu}_{2} \in \widetilde{h}_{2}\right\}$

(7) $\widetilde{h}_{1} \cap \widetilde{h}_{2}=\left\{\left[\min \left\{\mu_{1}^{-}, \mu_{2}^{-}\right\}, \min \left\{\mu_{1}^{+}, \mu_{2}^{+}\right\}\right]\right.$ $\left.\mid \widetilde{\mu}_{1} \in \widetilde{h}_{1}, \widetilde{\mu}_{2} \in \widetilde{h}_{2}\right\}$

Definition 6 (see [48]). Let $a=\left[a^{-}, a^{+}\right]$and $b=\left[b^{-}, b^{+}\right]$be two interval numbers, $\lambda>0$. The interval arithmetic is defined as
(1) $a+b=\left[a^{-}+b^{-}, a^{+}+b^{+}\right]$
(2) $a-b=\left[a^{-}-b^{+}, a^{+}-b^{-}\right]$
(3) $\lambda a=\left[\lambda a^{-}, \lambda a^{+}\right]$
(4) $a b=\left[\min \left\{a^{-} b^{-}, a^{-} b^{+}, a^{+} b^{-}, a^{+} b^{+}\right\}, \max \left\{a^{-} b^{-}\right.\right.$, $\left.\left.a^{-} b^{+}, a^{+} b^{-}, a^{+} b^{+}\right\}\right]$

Definition 7 (see [49]). Let $a=\left[a^{-}, a^{+}\right]$and $b=\left[b^{-}, b^{+}\right]$be two interval numbers and $l(a)=a^{+}-a^{-}$and $l(b)=b^{+}-b^{-}$, then the possibility degree of $a>b$ is defined as follows:

$$
P(a \geq b)=\max \left\{1-\max \left\{\frac{b^{+}-a^{-}}{l(a)+l(b)}, 0\right\}, 0\right\} .
$$

The equation of the possibility degree is used to compare two interval numbers. If $P(a \geq b)>0.5$, then $a$ is superior to $b$, denoted by $a>b$; if $P(a \geq b)=0.5$, then $a$ is equivalent to $b$, denoted by $a=b$.

Since an IVHFE is formed by several interval numbers, the comparative analysis of IVHFEs is different from that of HFEs. Based on the possibility degree of interval numbers in [49], Chen et al. [26] gave the following comparison laws.

Definition 8 (see [26]). For an IVHFE $\widetilde{h}, S(\widetilde{h})=1 /|\widetilde{h}| \sum_{\widetilde{\mu} \in \widetilde{h} \widetilde{\mu}}$ is called the score function of $\widetilde{h}$ with $|\cdot|$ denoting the cardinality, and $S(\widetilde{h})$ is an interval value belonging to $[0,1]$. For two IVHFEs, $\widetilde{h}_{1}$ and $\widetilde{h}_{2}$, if $P\left(S\left(\widetilde{h}_{1}\right) \geq S\left(\widetilde{h}_{2}\right)\right)>0.5$, then $\widetilde{h}_{1}$ is superior to $\widetilde{h}_{2}$, denoted by $\widetilde{h}_{1}>\widetilde{h}_{2}$.

2.3. Interval-Valued Pythagorean Fuzzy Set (IVPFS). A Pythagorean fuzzy set (PFS) is introduced by Yager [33], which is characterized by a membership function and a nonmembership function, where the sum of the square of the membership degree and the nonmembership degree of $x$ is less than or equal to 1 , while an intuitionistic fuzzy set is also characterized by them, where the sum is less than or equal to 1. Obviously, PFSs are more general than intuitionistic fuzzy sets. A PFS has emerged as an effective tool to solve multiattribute decision-making problems [37].

Definition 9 (see $[33,35])$. A Pythagorean fuzzy set (PFS) $P$ on $U$ is described as

$$
\begin{aligned}
P= & \left\{\left\langle x, \mu_{P}(x), v_{P}(x)\right\rangle \mid \mu_{P}^{2}(x)+v_{P}^{2}(x) \leq 1, \mu_{P}(x), \nu_{P}(x)\right. \\
& \in[0,1], x \in U\},
\end{aligned}
$$

where $\mu_{P}(x)$ and $\nu_{P}(x)$ represent the Pythagorean membership degree and the Pythagorean nonmembership degree of $\mathrm{P}$ at $x$, respectively.

Since people often find it difficult to exactly quantify their opinions facing with incomplete fuzzy decisionmaking problems, interval-valued fuzzy elements can provide a better solving way. Peng et al. [40] focused on intervalvalued Pythagorean fuzzy sets (IVPFSs), whose ideas are similar to interval-valued intuitionistic fuzzy sets.

Definition 10 (see [40]). An interval-valued Pythagorean fuzzy set (IVPFS) $\widetilde{P}$ on $U$ is described as

$$
\begin{aligned}
\widetilde{P}= & \left\{\left\langle x,\left[\mu_{\widetilde{P}}(x), \mu_{\widetilde{P}}^{ \pm}(x)\right],\left[\nu_{\widetilde{P}}(x), \nu_{\widetilde{P}}^{ \pm}(x)\right]\right\rangle \mid\left[\mu_{\widetilde{P}}(x), \mu_{\widetilde{P}}^{ \pm}(x)\right],\right. \\
& \left.\cdot\left[\nu_{\widetilde{P}}(x), v_{\widetilde{P}}^{ \pm}(x)\right] \in D[0,1],\left(\mu_{\widetilde{P}}^{ \pm}(x)\right)^{2}+\left(\nu_{\widetilde{P}}^{ \pm}(x)\right)^{2} \leq 1, x \in U\right\},
\end{aligned}
$$

where $\left[\mu_{\widetilde{P}}^{-}(x), \mu_{\widetilde{P}}^{+}(x)\right]$ and $\left[\nu_{\widetilde{P}}^{-}(x), \nu_{\widetilde{P}}^{+}(x)\right]$ are the Pythagorean membership intervals and the Pythagorean nonmembership intervals of $\widetilde{P}$ at $x$, respectively.

Each pair $\left\langle\left[\mu_{\widetilde{P}}^{-}(x), \mu_{\widetilde{P}}^{+}(x)\right],\left[\nu_{\widetilde{P}}^{-}(x), \nu_{\widetilde{P}}^{+}(x)\right]\right\rangle$ is called an interval-valued Pythagorean fuzzy element (IVPFE), denoted by $\widetilde{P}=\left\langle\left[\mu^{-}, \mu^{+}\right],\left[\nu^{-}, \nu^{+}\right]\right\rangle$. Obviously, for any IVPFE $\widetilde{P}$ on $U,\left[\mu^{-}, \mu^{+}\right]$and $\left[\nu^{-}, \nu^{+}\right]$are both singletons, the IVPFS degenerates into a PFS.

Definition 11 (see [40]). Let $\widetilde{P}=\left\langle\left[\mu^{-}, \mu^{+}\right],\left[\nu^{-}, \nu^{+}\right]\right\rangle$, $\widetilde{P}_{1}=\left\langle\left[\mu_{1}^{-}, \mu_{1}^{+}\right],\left[v_{1}^{-}, v_{1}^{+}\right]\right\rangle$, and $\widetilde{P}_{2}=\left\langle\left[\mu_{2}^{-}, \mu_{2}^{+}\right],\left[v_{2}^{-}, \nu_{2}^{+}\right]\right\rangle$be three IVPFEs and $\lambda>0$. The operational laws of IVPFEs are defined as follows:

(1) $\widetilde{P}^{C}=\left\langle\left[\nu^{-}, \nu^{+}\right],\left[\mu^{-}, \mu^{+}\right]\right\rangle$

(2) $\widetilde{P}^{\lambda}=\left\langle\left[\left(\mu^{-}\right)^{\lambda},\left(\mu^{+}\right)^{\lambda}\right],\left[\sqrt{1-\left(1-\left(\nu^{-}\right)^{2}\right)^{\lambda}}\right.\right.$,

$$
\left.\left.\sqrt{1-\left(1-\left(\nu^{+}\right)^{2}\right)^{\lambda}}\right]\right\rangle
$$

(3) $\lambda \widetilde{P}=\left\langle\left[\sqrt{1-\left(1-\left(\mu^{-}\right)^{2}\right)^{\lambda}}\right.\right.$,

$$
\left.\left.\sqrt{1-\left(1-\left(\mu^{+}\right)^{2}\right)^{\lambda}}\right],\left[\left(\nu^{-}\right)^{\lambda},\left(\nu^{+}\right)^{\lambda}\right]\right\rangle
$$

(4) $\widetilde{P}_{1} \oplus \widetilde{P}_{2}=\left\langle\left[\sqrt{\left(\mu_{1}^{-}\right)^{2}+\left(\mu_{2}^{-}\right)^{2}-\left(\mu_{1}^{-}\right)^{2}\left(\mu_{2}^{-}\right)^{2}}\right.\right.$,

$$
\left.\left.\sqrt{\left(\mu_{1}^{+}\right)^{2}+\left(\mu_{2}^{+}\right)^{2}-\left(\mu_{1}^{+}\right)^{2}\left(\mu_{2}^{+}\right)^{2}}\right],\left[\nu_{1}^{-} v_{2}^{-}, \nu_{1}^{+} \nu_{2}^{+}\right]\right\rangle
$$


(5) $\widetilde{P}_{1} \otimes \widetilde{P}_{2}=\left\langle\left[\mu_{1}^{-} \mu_{2}^{-}, \mu_{1}^{+} \mu_{2}^{+}\right],\left[\sqrt{\left(\nu_{1}^{-}\right)^{2}+\left(v_{2}^{-}\right)^{2}-\left(v_{1}^{-}\right)^{2}}\right.\right.$ $\left.\left.\left(v_{2}^{-}\right)^{2}, \sqrt{\left(\nu_{1}^{+}\right)^{2}+\left(v_{2}^{+}\right)^{2}-\left(\nu_{1}^{+}\right)^{2}\left(v_{2}^{+}\right)^{2}}\right]\right\rangle$

(6) $\widetilde{P}_{1} \cup \widetilde{P}_{2}=\left\langle\left[\max \left\{\mu_{1}^{-}, \mu_{2}^{-}\right\}, \max \left\{\mu_{1}^{+}, \mu_{2}^{+}\right\}\right]\right.$, $\left.\left[\min \left\{v_{1}^{-}, v_{2}^{-}\right\}, \min \left\{v_{1}^{+}, v_{2}^{+}\right\}\right]\right\rangle$

(7) $\widetilde{P}_{1} \cap \widetilde{P}_{2}=\left\langle\left[\min \left\{\mu_{1}^{-}, \mu_{2}^{-}\right\}, \min \left\{\mu_{1}^{+}, \mu_{2}^{+}\right\}\right],\left[\max \left\{v_{1}^{-}, v_{2}^{-}\right\}\right.\right.$, $\left.\left.\max \left\{v_{1}^{+}, v_{2}^{+}\right\}\right]\right\rangle$

Definition 12 (see [40]). For an IVPFE $\widetilde{P}=\left\langle\left[\mu^{-}, \mu^{+}\right]\right.$, $\left.\left[\nu^{-}, \nu^{+}\right]\right\rangle$, the score function of $\widetilde{P}$ is defined as follows:

$$
S(\widetilde{P})=\frac{1}{2}\left[\left(\mu^{-}\right)^{2}+\left(\mu^{+}\right)^{2}-\left(\nu^{-}\right)^{2}-\left(\nu^{+}\right)^{2}\right] .
$$

The accuracy function of $\widetilde{P}$ is defined as follows:

$$
H(\widetilde{P})=\frac{1}{2}\left[\left(\mu^{-}\right)^{2}+\left(\mu^{+}\right)^{2}+\left(\nu^{-}\right)^{2}+\left(\nu^{+}\right)^{2}\right] .
$$

For two IVPFEs $\widetilde{P}_{1}$ and $\widetilde{P}_{2}$,

(1) If $S\left(\widetilde{P}_{1}\right)>S\left(\widetilde{P}_{2}\right)$, then $\widetilde{P}_{1}>\widetilde{P}_{2}$

(2) If $S\left(\widetilde{P}_{1}\right)=S\left(\widetilde{P}_{2}\right)$, then

(2.1) If $H\left(\widetilde{P}_{1}\right)>H\left(\widetilde{P}_{2}\right)$, then $\widetilde{P}_{1}>\widetilde{P}_{2}$;

(2.2) If $H\left(\widetilde{P}_{1}\right)=H\left(\widetilde{P}_{2}\right)$, then $\widetilde{P}_{1}=\widetilde{P}_{2}$.

\section{Interval-Valued Pythagorean Hesitant Fuzzy Sets (IVPHFSs)}

3.1. Interval-Valued Pythagorean Hesitant Fuzzy Elements. As mentioned earlier, in many practical problems, it is difficult for decision makers to determine precise membership degrees or nonmembership degrees, and the evaluation with relatively reasonable interval values often exists in decision-making. In order to better avoid the information loss and enhance the flexibility and applicability of the decision-making models in dealing with qualitative information, we propose the concept of interval-valued Pythagorean hesitant fuzzy set (IVPHFS).

Definition 13. An interval-valued Pythagorean hesitant fuzzy set (IVPHFS) $\mathscr{P}$ on $U$ is described as

$$
\mathscr{P}=\left\{\left\langle x, h_{\mathscr{P}}(x)\right\rangle \mid x \in U\right\},
$$

where

$$
\begin{aligned}
& h_{\mathscr{P}}(x)=\left\{\left\langle\widetilde{\mu}_{\mathscr{P}}(x), \tilde{\nu}_{\mathscr{P}}(x)\right\rangle \mid \tilde{\mu}_{\mathscr{P}}(x)=\left[\mu_{\mathscr{P}}^{-}(x), \mu_{\mathscr{P}}^{+}(x)\right] \in D[0,1],\right. \\
& \left.\tilde{v}_{\mathscr{P}}(x)=\left[\nu_{\mathscr{P}}^{-}(x), \nu_{\mathscr{P}}^{+}(x)\right] \in D[0,1],\left(\mu_{\mathscr{P}}^{+}(x)\right)^{2}+\left(\nu_{\mathscr{P}}^{+}(x)\right)^{2} \leq 1\right\},
\end{aligned}
$$

where $\widetilde{\mu}_{\mathscr{P}}(x)$ and $\widetilde{\nu}_{\mathscr{P}}(x)$ are the possible Pythagorean membership intervals and the possible Pythagorean nonmembership intervals of $\mathscr{P}$ at $x$, respectively. The set of all IVPHFEs on $U$ is denoted by $\Omega$.

Obviously, for each $x \in U$, if $h_{\mathscr{P}}(x)$ includes only one pair of intervals, the IVPHFS degenerates into an IVPFS; if both $\widetilde{\mu}_{\mathscr{P}}(x)$ and $\widetilde{v}_{\mathscr{P}}(x)$ degenerate one singleton, the IVPHFS can be seen as a PHFS; if $\tilde{v}_{\mathscr{P}}(x)=[0,0]$, the IVPHFS can be seen as an IVHFS; if $\mu_{\mathscr{P}}^{+}(x)+\nu_{\mathscr{P}}^{+}(x) \leq 1$, the
IVPHFS can be seen as an interval-valued intuitionistic hesitant fuzzy set [29].

For convenience, we call each pair $\widetilde{P}=h_{\mathscr{P}}(x)$ as an interval-valued Pythagorean hesitant fuzzy element (IVPHFE), where $\widetilde{\mathscr{P}}=\left\{\langle\widetilde{\mu}, \widetilde{\nu}\rangle \mid \widetilde{\mu}=\left[\mu^{-}, \mu^{+}\right], \widetilde{\nu}=\left[\nu^{-}, \nu^{+}\right]\right\}$.

Based on the operators of IVHFEs [27] and IVPFEs [40], the operational laws of IVPHFEs are defined as follows.

Definition 14. Let $\widetilde{\mathscr{P}}=\left\{\langle\widetilde{\mu}, \widetilde{\nu}\rangle \mid \widetilde{\mu}=\left[\mu^{-}, \mu^{+}\right], \widetilde{\nu}=\left[\nu^{-}, \nu^{+}\right]\right\}, \widetilde{\mathscr{P}}_{1}=$ $\left\{\left\langle\widetilde{\mu}_{1}, \widetilde{\nu}_{1}\right\rangle \mid \widetilde{\mu}_{1}=\left[\mu_{1}^{-}, \mu_{1}^{+}\right], \widetilde{\nu}_{1}=\left[v_{1}^{-}, v_{1}^{+}\right]\right\}$, and $\widetilde{P}_{2}=\left\{\left\langle\widetilde{\mu}_{2}, \widetilde{\nu}_{2}\right\rangle \mid \widetilde{\mu}_{2}=\right.$ $\left.\left[\mu_{2}^{-}, \mu_{2}^{+}\right], \widetilde{\nu}_{2}=\left[v_{2}^{-}, \nu_{2}^{+}\right]\right\}$be three IVPHFEs and $\lambda>0$. The operational laws of IVPHFEs are defined as follows:

(1) $\widetilde{\mathscr{P}}^{C}=\left\{\left\langle\left[\nu^{-}, \nu^{+}\right],\left[\mu^{-}, \mu^{+}\right]\right\rangle \mid\langle\widetilde{\mu}, \widetilde{\nu}\rangle \in \widetilde{\mathscr{P}}\right\}$

(2) $\widetilde{\mathscr{P}}^{\lambda}=\left\{\left\langle\left[\left(\mu^{-}\right)^{\lambda},\left(\mu^{+}\right)^{\lambda}\right],\left[\sqrt{1-\left(1-\left(\nu^{-}\right)^{2}\right)^{\lambda}}\right.\right.\right.$, $\left.\left.\left.\sqrt{1-\left(1-\left(\nu^{+}\right)^{2}\right)^{\lambda}}\right]\right\rangle \mid\langle\tilde{\mu}, \tilde{\nu}\rangle \in \widetilde{\mathscr{P}}\right\}$

(3) $\lambda \widetilde{\mathscr{P}}=\left\{\left[\sqrt{1-\left(1-\left(\mu^{-}\right)^{2}\right)^{\lambda}}, \sqrt{1-\left(1-\left(\mu^{+}\right)^{2}\right)^{\lambda}}\right]\right.$, $\left.\left\langle\left[\left(\nu^{-}\right)^{\lambda},\left(\nu^{+}\right)^{\lambda}\right]\right\rangle \mid\langle\widetilde{\mu}, \widetilde{\nu}\rangle \in \widetilde{\mathscr{P}}\right\}$

(4) $\widetilde{\mathscr{P}}_{1} \oplus \widetilde{\mathscr{P}}_{2}=\left\{\left\langle\left[\sqrt{\left(\mu_{1}^{-}\right)^{2}+\left(\mu_{2}^{-}\right)^{2}-\left(\mu_{1}^{-}\right)^{2}\left(\mu_{2}^{-}\right)^{2}}\right.\right.\right.$, $\left.\sqrt{\left(\mu_{1}^{+}\right)^{2}+\left(\mu_{2}^{+}\right)^{2}-\left(\mu_{1}^{+}\right)^{2}\left(\mu_{2}^{+}\right)^{2}}\right]$, $\left.\left.\left[v_{1}^{-} v_{2}^{-}, v_{1}^{+} \nu_{2}^{+}\right]\right\rangle \mid\left\langle\widetilde{\mu}_{i}, \widetilde{v}_{i}\right\rangle \in \widetilde{\mathscr{P}}_{i}, i=1,2\right\}$

(5) $\widetilde{\mathscr{P}}_{1} \otimes \widetilde{\mathscr{P}}_{2}=\left\{\left\langle\left[\mu_{1}^{-} \mu_{2}^{-}, \mu_{1}^{+} \mu_{2}^{+}\right]\left[{\sqrt{\left(v_{1}^{-}\right)}}^{2}+\left(v_{2}^{-}\right)^{2}-\left(v_{1}^{-}\right)^{2}\right.\right.\right.$ $\left.\left.\left(v_{2}^{-}\right)^{2}, \sqrt{\left(v_{1}^{+}\right)^{2}+\left(v_{2}^{+}\right)^{2}-\left(v_{1}^{+}\right)^{2}\left(v_{2}^{+}\right)^{2}}\right]\right\rangle, \quad \mid\left\langle\widetilde{\mu}_{i}, \widetilde{v}_{i}\right\rangle \epsilon$ $\left.\widetilde{\mathscr{P}}_{i}, i=1,2\right\}$

(6) $\widetilde{\mathscr{P}}_{1} \cup \widetilde{\mathfrak{P}}_{2}=\left\{\left\langle\left[\max \left\{\mu_{1}^{-}, \mu_{2}^{-}\right\}, \max \left\{\mu_{1}^{+}, \mu_{2}^{+}\right\}\right]\right.\right.$, $\left.\left.\left[\min \left\{v_{1}^{-}, v_{2}^{-}\right\}, \min \left\{v_{1}^{+}, v_{2}^{+}\right\}\right]\right\rangle \mid\left\langle\widetilde{\mu}_{i}, \widetilde{v}_{i}\right\rangle \in \widetilde{\mathscr{P}}_{i}, i=1,2\right\}$

(7) $\widetilde{\mathscr{P}}_{1} \cap \widetilde{\mathscr{P}}_{2}=\left\{\left\langle\left[\min \left\{\mu_{1}^{-}, \mu_{2}^{-}\right\}, \min \left\{\mu_{1}^{+}, \mu_{2}^{+}\right\}\right]\right.\right.$, $\left.\left.\left[\max \left\{v_{1}^{-}, v_{2}^{-}\right\}, \max \left\{v_{1}^{+}, v_{2}^{+}\right\}\right]\right\rangle \mid\left\langle\widetilde{\mu}_{i}, \widetilde{v}_{i}\right\rangle \in \widetilde{\mathscr{P}}_{i}, i=1,2\right\}$

Proposition 1. Let $\widetilde{\mathscr{P}}, \widetilde{\mathscr{P}}_{1}$, and $\widetilde{\mathscr{P}}_{2}$ be three IVPHFEs and $\lambda>0$, then $\widetilde{\mathscr{P}}^{\mathrm{C}}, \widetilde{\mathscr{P}}^{\lambda}, \lambda \widetilde{\mathscr{P}}, \widetilde{\mathscr{P}}_{1} \oplus \widetilde{\mathscr{P}}_{2}, \widetilde{\mathscr{P}}_{1} \otimes \widetilde{\mathscr{P}}_{2}, \widetilde{\mathscr{P}}_{1} \cup \widetilde{\mathscr{P}}_{2}$, and $\widetilde{\mathfrak{P}}_{1} \cap \widetilde{\mathfrak{P}}_{2}$ are all IVPHFESs.

Proof. Obviously, $\widetilde{\mathscr{P}}^{\mathrm{C}}$ is an IVPHFE.

For any $\langle\widetilde{\mu}, \widetilde{\nu}\rangle \in \widetilde{\mathscr{P}}$, since $\left(\mu^{+}\right)^{2}+\left(\nu^{+}\right)^{2} \leq 1$,

$$
\begin{aligned}
\left(\left(\mu^{+}\right)^{\lambda}\right)^{2}+\left(\sqrt{1-\left(1-\left(\nu^{+}\right)^{2}\right)^{\lambda}}\right)^{2} & =\left(\mu^{+}\right)^{2 \lambda}+1-\left(1-\left(\nu^{+}\right)^{2}\right)^{\lambda} \\
& \leq\left(\mu^{+}\right)^{2 \lambda}+1-\left(\mu^{+}\right)^{2 \lambda}=1 .
\end{aligned}
$$

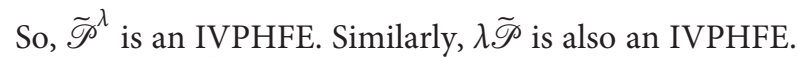
As for $\widetilde{\mathfrak{P}}_{1} \oplus \widetilde{\mathscr{P}}_{2}$,

$$
\begin{aligned}
\left(\mu_{1}^{+}\right)^{2} & +\left(\mu_{2}^{+}\right)^{2}-\left(\mu_{1}^{+}\right)^{2}\left(\mu_{2}^{+}\right)^{2}+\left(v_{1}^{+}\right)^{2}\left(v_{2}^{+}\right)^{2} \\
\leq & \left(\mu_{1}^{+}\right)^{2}+\left(\mu_{2}^{+}\right)^{2}-\left(\mu_{1}^{+}\right)^{2}\left(\mu_{2}^{+}\right)^{2}+\left(1-\left(\mu_{1}^{+}\right)^{2}\right)\left(1-\left(v_{2}^{+}\right)^{2}\right) \\
= & \left(\mu_{1}^{+}\right)^{2}+\left(\mu_{2}^{+}\right)^{2}-\left(\mu_{1}^{+}\right)^{2}\left(\mu_{2}^{+}\right)^{2}+1-\left(\mu_{1}^{+}\right)^{2}-\left(\mu_{2}^{+}\right)^{2} \\
& +\left(\mu_{1}^{+}\right)^{2}\left(\mu_{2}^{+}\right)^{2}=1 .
\end{aligned}
$$


So, $\widetilde{\mathscr{P}}_{1} \oplus \widetilde{\mathscr{P}}_{2}$ is an IVPHFE. Similarly, $\widetilde{\mathscr{P}}_{1} \otimes \widetilde{\mathscr{P}}_{2}$ is also an IVPHFE.

At last, we prove the last two claims. Assume $\mu_{1}^{+}<\mu_{2}^{+}$.

Then, $\left(\max \left\{\mu_{1}^{+}, \mu_{2}^{+}\right\}\right)^{2}=\left(\mu_{2}^{+}\right)^{2} \leq 1-\left(\nu_{2}^{+}\right)^{2} \leq 1-\left(\min \left\{v_{1}^{+}\right.\right.$, $\left.\left.v_{2}^{+}\right\}\right)^{2}$. So, $\widetilde{\mathfrak{P}}_{1} \cup \widetilde{\mathscr{P}}_{2}$ is an IVPHFE. Similarly, $\widetilde{\mathscr{P}}_{1} \cap \widetilde{\mathscr{P}}_{2}$ is also an IVPHFE. All the claims are proved.

Proposition 2. Let $\widetilde{\mathscr{P}}, \widetilde{\mathscr{P}}_{1}, \widetilde{\mathscr{P}}_{2}$, and $\widetilde{\mathscr{P}}_{3}$ be four IVPHFEs and $\lambda, \lambda_{1}, \lambda_{2}>0$, then

(1) $\widetilde{\mathscr{P}}_{1} \oplus \widetilde{\mathscr{P}}_{2}=\widetilde{\mathscr{P}}_{2} \oplus \widetilde{\mathscr{P}}_{1}$ and $\widetilde{\mathscr{P}}_{1} \otimes \widetilde{\mathscr{P}}_{2}=\widetilde{\mathscr{P}}_{2} \otimes \widetilde{\mathscr{P}}_{1}$

(2) $\lambda \widetilde{\mathscr{P}}_{1} \oplus \lambda \widetilde{\mathscr{P}}_{2}=\lambda\left(\widetilde{\mathscr{P}}_{1} \oplus \widetilde{\mathscr{P}}_{2}\right)$ and $\left(\widetilde{\mathscr{P}}_{1}\right)^{\lambda} \otimes\left(\widetilde{\mathscr{P}}_{2}\right)^{\lambda}=\left(\widetilde{\mathscr{P}}_{1}\right.$ $\left.\otimes \mathscr{\mathscr { P }}_{2}\right)^{\lambda}$
(3) $\lambda_{1} \widetilde{\mathscr{P}} \oplus \lambda_{2} \widetilde{\mathscr{P}}=\left(\lambda_{1}+\lambda_{2}\right) \widetilde{\mathscr{P}}$ and $\widetilde{\mathscr{P}}^{\lambda_{1}} \otimes \widetilde{\mathscr{P}}^{\lambda_{2}}=\widetilde{\mathscr{P}}^{\left(\lambda_{1}+\lambda_{2}\right)}$

(4) $\left(\widetilde{\mathscr{P}}^{C}\right)^{\lambda}=(\lambda \widetilde{\mathscr{P}})^{C}$ and $\lambda\left(\widetilde{\mathscr{P}}^{C}\right)=\left(\widetilde{\mathscr{P}}^{\lambda}\right)^{C}$

(5) $\left(\widetilde{\mathscr{P}}_{1}\right)^{C} \oplus\left(\widetilde{\mathscr{P}}_{2}\right)^{C}=\left(\widetilde{\mathscr{P}}_{1} \otimes \widetilde{\mathscr{P}}_{2}\right)^{C}$ and $\left(\widetilde{\mathscr{P}}_{1}\right)^{C} \otimes\left(\widetilde{\mathscr{P}}_{2}\right)^{C}=$ $\left(\widetilde{\mathscr{P}}_{1} \oplus \widetilde{\mathscr{P}}_{2}\right)^{\mathrm{C}}$

(6) $\left(\widetilde{\mathscr{P}}_{1} \oplus \widetilde{\mathscr{P}}_{2}\right) \oplus \widetilde{\mathscr{P}}_{3}=\widetilde{\mathscr{P}}_{1} \oplus\left(\widetilde{\mathscr{P}}_{2} \oplus \widetilde{\mathscr{P}}_{3}\right)$ and $\left(\widetilde{\mathscr{P}}_{1} \otimes \widetilde{\mathscr{P}}_{2}\right)$ $\otimes \mathscr{P}_{3}=\mathscr{P}_{1} \otimes\left(\mathscr{P}_{2} \otimes \mathscr{P}_{3}\right)$

Proof. (1) Based on Definition 14, claim (1) is obvious, so here the proof process is overleaped.

$$
\begin{aligned}
\lambda \widetilde{\mathscr{P}}_{1} \oplus \lambda \widetilde{\mathscr{P}}_{2}= & \left.\left\{\left\langle\sqrt{1-\left(1-\left(\mu_{1}^{-}\right)^{2}\right)^{\lambda}}, \sqrt{1-\left(1-\left(\mu_{1}^{+}\right)^{2}\right)^{\lambda}}\right],\left[\left(v_{1}^{-}\right)^{\lambda},\left(v_{1}^{+}\right)^{\lambda}\right]\right\rangle \mid\left\langle\widetilde{\mu}_{1}, \widetilde{v}_{1}\right\rangle \in \widetilde{\mathscr{P}}_{1}\right\} \\
& \left.\oplus\left\{\left\langle\sqrt{1-\left(1-\left(\mu_{2}^{-}\right)^{2}\right)^{\lambda}}, \sqrt{1-\left(1-\left(\mu_{2}^{+}\right)^{2}\right)^{\lambda}}\right],\left[\left(v_{2}^{-}\right)^{\lambda},\left(v_{2}^{+}\right)^{\lambda}\right]\right\rangle \mid\left\langle\widetilde{\mu}_{2}, \widetilde{v}_{2}\right\rangle \in \widetilde{\mathscr{P}}_{2}\right\} \\
= & \left.\left\{\left\langle\sqrt{1-\left(1-\left(\mu_{1}^{-}\right)^{2}\right)^{\lambda}\left(1-\left(\mu_{2}^{-}\right)^{2}\right)^{\lambda}}, \sqrt{1-\left(1-\left(\mu_{1}^{+}\right)^{2}\right)^{\lambda}\left(1-\left(\mu_{2}^{+}\right)^{2}\right)^{\lambda}}\right],\left[\left(\nu_{1}^{-} v_{2}^{-}\right)^{\lambda},\left(v_{1}^{+} v_{2}^{+}\right)^{\lambda}\right]\right\rangle \mid\left\langle\widetilde{\mu}_{i}, \widetilde{v}_{i}\right\rangle \in \widetilde{\mathscr{P}}_{i}, i=1,2\right\}, \\
& \lambda\left(\widetilde{\mathscr{P}}_{1} \oplus \widetilde{\mathscr{P}}_{2}\right) \\
= & \left.\lambda\left\{\left\langle\sqrt{\left(\mu_{1}^{-}\right)^{2}+\left(\mu_{2}^{-}\right)^{2}-\left(\mu_{1}^{-}\right)^{2}\left(\mu_{2}^{-}\right)^{2}}, \sqrt{\left(\mu_{1}^{+}\right)^{2}+\left(\mu_{2}^{+}\right)^{2}-\left(\mu_{1}^{+}\right)^{2}\left(\mu_{2}^{+}\right)^{2}}\right],\left[v_{1}^{-} v_{2}^{-}, v_{1}^{+} v_{2}^{+}\right]\right\rangle \mid\left\langle\widetilde{\mu}_{i}, \widetilde{v}_{i}\right\rangle \in \widetilde{\mathscr{P}}_{i}, i=1,2\right\} \\
= & \left.\lambda\left\{\left\langle\sqrt{1-\left(1-\left(\mu_{1}^{-}\right)^{2}\right)\left(1-\left(\mu_{2}^{-}\right)^{2}\right)}, \sqrt{1-\left(1-\left(\mu_{1}^{+}\right)^{2}\right)\left(1-\left(\mu_{2}^{+}\right)^{2}\right)}\right],\left[v_{1}^{-} v_{2}^{-}, v_{1}^{+} v_{2}^{+}\right]\right\rangle \mid\left\langle\widetilde{\mu}_{i}, \widetilde{v}_{i}\right\rangle \in \widetilde{\mathscr{P}}_{i}, i=1,2\right\} \\
= & \left.\left\{\left\langle\sqrt{1-\left(1-\left(\mu_{1}^{-}\right)^{2}\right)^{\lambda}\left(1-\left(\mu_{2}^{-}\right)^{2}\right)^{\lambda}}, \sqrt{1-\left(1-\left(\mu_{1}^{+}\right)^{2}\right)^{\lambda}\left(1-\left(\mu_{2}^{+}\right)^{2}\right)^{\lambda}}\right],\left[\left(v_{1}^{-} v_{2}^{-}\right)^{\lambda},\left(v_{1}^{+} v_{2}^{+}\right)^{\lambda}\right]\right\rangle \mid\left\langle\widetilde{\mu}_{i}, \widetilde{v}_{i}\right\rangle \in \widetilde{\mathscr{P}}_{i}, i=1,2\right\} .
\end{aligned}
$$

So, $\lambda \widetilde{\mathscr{P}}_{1} \oplus \lambda \widetilde{\mathscr{P}}_{2}=\lambda\left(\widetilde{\mathscr{P}}_{1} \oplus \widetilde{\mathscr{P}}_{2}\right)$ holds. Similarly, we have $\left(\widetilde{\mathscr{P}}_{1}\right)^{\lambda} \otimes\left(\widetilde{\mathscr{P}}_{2}\right)^{\lambda}=\left(\widetilde{\mathscr{P}}_{1} \otimes \widetilde{\mathscr{P}}_{2}\right)^{\lambda}$.

$$
\begin{aligned}
\lambda_{1} \widetilde{P}_{\oplus} \lambda_{2} \widetilde{\mathscr{P}} & =\left\{\left\langle\left[\sqrt{1-\left(1-\left(\mu^{-}\right)^{2}\right)^{\lambda_{1}}}, \sqrt{1-\left(1-\left(\mu^{+}\right)^{2}\right)^{\lambda_{1}}}\right],\left[\left(\nu^{-}\right)^{\lambda_{1}},\left(\nu^{+}\right)^{\lambda_{1}}\right]\right\rangle \mid\langle\widetilde{\mu}, \widetilde{\nu}\rangle \in \widetilde{\mathscr{P}}\right\} \\
& \left.\oplus\left\{\left\langle\sqrt{1-\left(1-\left(\mu^{-}\right)^{2}\right)^{\lambda_{2}}}, \sqrt{1-\left(1-\left(\mu^{+}\right)^{2}\right)^{\lambda_{2}}}\right],\left[\left(\nu^{-}\right)^{\lambda_{2}},\left(\nu^{+}\right)^{\lambda_{2}}\right]\right\rangle \mid\langle\tilde{\mu}, \widetilde{\nu}\rangle \in \widetilde{\mathscr{P}}\right\} \\
& =\left\{\left\langle\left[\sqrt{1-\left(1-\left(\mu^{-}\right)^{2}\right)^{\left(\lambda_{1}+\lambda_{2}\right)}}, \sqrt{1-\left(1-\left(\mu^{+}\right)^{2}\right)^{\left(\lambda_{1}+\lambda_{2}\right)}}\right],\left[\left(\nu^{-}\right)^{\left(\lambda_{1}+\lambda_{2}\right)},\left(\nu^{+}\right)^{\left(\lambda_{1}+\lambda_{2}\right)}\right]\right\rangle \mid\langle\widetilde{\mu}, \widetilde{\nu}\rangle \in \widetilde{\mathscr{P}}\right\} \\
& =\left(\lambda_{1}+\lambda_{2}\right) \widetilde{\mathscr{P}} .
\end{aligned}
$$

Similarly, we have $\widetilde{\mathscr{P}}^{\lambda_{1}} \otimes \widetilde{\mathscr{P}}^{\lambda_{2}}=\widetilde{\mathscr{P}}^{\left(\lambda_{1}+\lambda_{2}\right)}$. 


$$
\begin{aligned}
\left(\widetilde{\mathscr{P}}^{C}\right)^{\lambda} & =\left\{\left\langle\left[\left(\nu^{-}\right)^{\lambda},\left(\nu^{+}\right)^{\lambda}\right],\left[\sqrt{1-\left(1-\left(\mu^{-}\right)^{2}\right)^{\lambda}}, \sqrt{1-\left(1-\left(\mu^{+}\right)^{2}\right)^{\lambda}}\right]\right\rangle \mid\langle\widetilde{\mu}, \widetilde{\nu}\rangle \in \widetilde{\mathscr{P}}\right\} \\
& =\left\{\left\langle\left[\sqrt{1-\left(1-\left(\mu^{-}\right)^{2}\right)^{\lambda}}, \sqrt{1-\left(1-\left(\mu^{+}\right)^{2}\right)^{\lambda}}\right],\left[\left(\nu^{-}\right)^{\lambda},\left(\nu^{+}\right)^{\lambda}\right]\right\rangle \mid\langle\tilde{\mu}, \widetilde{\nu}\rangle \in \tilde{\mathscr{P}}\right\} \\
& =(\lambda \tilde{\mathscr{P}})^{C} .
\end{aligned}
$$

Similarly, we have $\lambda\left(\widetilde{\mathscr{P}}^{C}\right)=\left(\tilde{\mathscr{P}}^{\lambda}\right)^{C}$.

$$
\begin{aligned}
& \widetilde{\mathscr{P}}_{1}^{C} \oplus \widetilde{\mathscr{P}}_{2}^{C}=\left\{\left\langle\left[\nu_{1}^{-}, v_{1}^{+}\right],\left[\mu_{1}^{-}, \mu_{1}^{+}\right]\right\rangle \mid\left\langle\widetilde{\mu}_{1}, \widetilde{v}_{1}\right\rangle \in \widetilde{\mathscr{P}}_{1}\right\} \oplus\left\{\left\langle\left[v_{2}^{-}, v_{2}^{+}\right],\left[\mu_{2}^{-}, \mu_{2}^{+}\right]\right\rangle \mid\left\langle\widetilde{\mu}_{2}, \widetilde{v}_{2}\right\rangle \in \widetilde{\mathscr{P}}_{2}\right\} \\
& =\left\{\left\langle\left[\sqrt{\left(v_{1}^{-}\right)^{2}+\left(v_{2}^{-}\right)^{2}-\left(v_{1}^{-}\right)^{2}\left(v_{2}^{-}\right)^{2}}, \sqrt{\left(\nu_{1}^{+}\right)^{2}+\left(\nu_{2}^{+}\right)^{2}-\left(v_{1}^{+}\right)^{2}\left(v_{2}^{+}\right)^{2}}\right],\left[\mu_{1}^{-} \mu_{2}^{-}, \mu_{1}^{+} \mu_{2}^{+}\right]\right\rangle \mid\left\langle\widetilde{\mu}_{i}, \widetilde{v}_{i}\right\rangle \in \widetilde{\mathscr{P}}_{i}, i=1,2\right\} \\
& =\left\{\left\langle\left[\mu_{1}^{-} \mu_{2}^{-}, \mu_{1}^{+} \mu_{2}^{+}\right],\left[\sqrt{\left(v_{1}^{-}\right)^{2}+\left(v_{2}^{-}\right)^{2}-\left(v_{1}^{-}\right)^{2}\left(v_{2}^{-}\right)^{2}}, \sqrt{\left(\nu_{1}^{+}\right)^{2}+\left(v_{2}^{+}\right)^{2}-\left(v_{1}^{+}\right)^{2}\left(v_{2}^{+}\right)^{2}}\right]\right\rangle \mid\left\langle\widetilde{\mu}_{i}, \widetilde{v}_{i}\right\rangle \in \widetilde{\mathscr{P}}_{i}, i=1,2\right\} \\
& =\left(\widetilde{\mathscr{P}}_{1} \otimes \widetilde{\mathscr{P}}_{2}\right)^{C} \text {. }
\end{aligned}
$$

Similarly, $\widetilde{\mathscr{P}}_{1}^{C} \otimes \widetilde{\mathscr{P}}_{2}^{C}=\left(\widetilde{\mathscr{P}}_{1} \oplus \widetilde{\mathscr{P}}_{2}\right)^{C}$ holds.

$$
\begin{aligned}
& \left(\widetilde{\mathscr{P}}_{1} \oplus \widetilde{\mathscr{P}}_{2}\right) \oplus \widetilde{\mathscr{P}}_{3}=\left\{\left\langle\left[\sqrt{\left(\mu_{1}^{-}\right)^{2}+\left(\mu_{2}^{-}\right)^{2}-\left(\mu_{1}^{-}\right)^{2}\left(\mu_{2}^{-}\right)^{2}}, \sqrt{\left(\mu_{1}^{+}\right)^{2}+\left(\mu_{2}^{+}\right)^{2}-\left(\mu_{1}^{+}\right)^{2}\left(\mu_{2}^{+}\right)^{2}}\right],\left[\nu_{1}^{-} v_{2}^{-}, v_{1}^{+} v_{2}^{+}\right]\right\rangle \mid\left\langle\widetilde{\mu}_{1}, \widetilde{v}_{1}\right\rangle \in \widetilde{\mathscr{P}}_{1},\left\langle\widetilde{\mu}_{2}, \widetilde{v}_{2}\right\rangle \in \widetilde{\mathscr{P}}_{2}\right\} \\
& \oplus\left\{\left\langle\left[\mu_{3}^{-}, \mu_{3}^{+}\right],\left[\nu_{3}^{-}, v_{3}^{+}\right]\right\rangle \mid\left\langle\widetilde{\mu}_{3}, \widetilde{v}_{3}\right\rangle \in \widetilde{\mathscr{P}}_{3}\right\} \\
& =\left\{\left\langle\left[\sqrt{\left(\mu_{1}^{-}\right)^{2}+\left(\mu_{2}^{-}\right)^{2}+\left(\mu_{3}^{-}\right)^{2}-\left(\mu_{1}^{-}\right)^{2}\left(\mu_{2}^{-}\right)^{2}-\left(\mu_{1}^{-}\right)^{2}\left(\mu_{3}^{-}\right)^{2}-\left(\mu_{2}^{-}\right)^{2}\left(\mu_{3}^{-}\right)^{2}+\left(\mu_{1}^{-}\right)^{2}\left(\mu_{2}^{-}\right)^{2}\left(\mu_{3}^{-}\right)^{2}},\right.\right.\right. \\
& \left.\sqrt{\left(\mu_{1}^{+}\right)^{2}+\left(\mu_{2}^{+}\right)^{2}+\left(\mu_{3}^{+}\right)^{2}-\left(\mu_{1}^{+}\right)^{2}\left(\mu_{2}^{+}\right)^{2}-\left(\mu_{1}^{+}\right)^{2}\left(\mu_{3}^{+}\right)^{2}-\left(\mu_{2}^{+}\right)^{2}\left(\mu_{3}^{+}\right)^{2}+\left(\mu_{1}^{+}\right)^{2}\left(\mu_{2}^{+}\right)^{2}\left(\mu_{3}^{+}\right)^{2}}\right] \\
& \left.\left.\left[v_{1}^{-} v_{2}^{-} v_{3}^{-}, v_{1}^{+} v_{2}^{+} v_{3}^{+}\right]\right\rangle \mid\left\langle\widetilde{\mu}_{i}, \widetilde{v}_{i}\right\rangle \in \widetilde{\mathscr{P}}_{i}, i=1,2,3\right\} \\
& =\left\{\left\langle\left[\mu_{1}^{-}, \mu_{1}^{+}\right],\left[\nu_{1}^{-}, \nu_{1}^{+}\right]\right\rangle \mid\left\langle\widetilde{\mu}_{1}, \widetilde{v}_{1}\right\rangle \in \widetilde{\mathscr{P}}_{1}\right\} \oplus\left\{\left\langle\left[\sqrt{\left(\mu_{2}^{-}\right)^{2}+\left(\mu_{3}^{-}\right)^{2}-\left(\mu_{2}^{-}\right)^{2}\left(\mu_{3}^{-}\right)^{2}}, \sqrt{\left(\mu_{2}^{+}\right)^{2}+\left(\mu_{3}^{+}\right)^{2}-\left(\mu_{2}^{+}\right)^{2}\left(\mu_{3}^{+}\right)^{2}}\right]\right.\right. \text {, } \\
& \left.\left.\left[\nu_{2}^{-} v_{3}^{-}, v_{2}^{+} v_{3}^{+}\right]\right\rangle \mid\left\langle\widetilde{\mu}_{i}, \widetilde{v}_{i}\right\rangle \in \widetilde{\mathscr{P}}_{i}, i=2,3\right\} \\
& =\widetilde{\mathscr{P}}_{1} \oplus\left(\widetilde{\mathscr{P}}_{2} \oplus \widetilde{\mathscr{P}}_{3}\right) \text {. }
\end{aligned}
$$

Similarly, we have $\left(\widetilde{\mathscr{P}}_{1} \otimes \widetilde{\mathscr{P}}_{2}\right) \otimes \widetilde{\mathscr{P}}_{3}=\widetilde{\mathscr{P}}_{1} \otimes\left(\widetilde{\mathscr{P}}_{2} \otimes \widetilde{\mathscr{P}}_{3}\right)$. All the claims of the proposition are proved.

Proposition 3. Let $\widetilde{\mathscr{P}}, \widetilde{\mathscr{P}}_{1}, \widetilde{\mathscr{P}}_{2}$, and $\widetilde{\mathscr{P}}_{3}$ be four IVPHFEs and $\lambda>0$, then

(1) $\widetilde{\mathscr{P}}_{1} \cup \widetilde{\mathscr{P}}_{2}=\widetilde{\mathscr{P}}_{2} \cup \widetilde{\mathscr{P}}_{1}$ and $\widetilde{\mathscr{P}}_{1} \cap \widetilde{\mathscr{P}}_{2}=\widetilde{\mathscr{P}}_{2} \cap \widetilde{\mathscr{P}}_{1}$

(2) $\lambda \widetilde{\mathscr{P}}_{1} \cup \lambda \widetilde{\mathscr{P}}_{2}=\lambda\left(\widetilde{\mathscr{P}}_{1} \cup \widetilde{\mathscr{P}}_{2}\right)$ and $\left(\widetilde{\mathscr{P}}_{1}\right)^{\lambda} \cap\left(\widetilde{\mathscr{P}}_{2}\right)^{\lambda}=\left(\widetilde{\mathscr{P}}_{1}\right.$ $\left.\cap \widetilde{\mathscr{P}}_{2}\right)^{\lambda}$
(3) $\left(\tilde{\mathscr{P}}_{1}\right)^{C} \cup\left(\widetilde{\mathscr{P}}_{2}\right)^{C}=\left(\widetilde{\mathscr{P}}_{1} \cap \widetilde{\mathscr{P}}_{2}\right)^{C}$ and $\left(\tilde{\mathscr{P}}_{1}\right)^{C} \cap\left(\tilde{\mathscr{P}}_{2}\right)^{C}$ $=\left(\widetilde{\mathscr{P}}_{1} \cup \widetilde{\mathscr{P}}_{2}\right)^{\mathrm{C}}$

(4) $\left(\widetilde{\mathscr{P}}_{1} \cup \widetilde{\mathscr{P}}_{2}\right) \cup \widetilde{\mathscr{P}}_{3}=\widetilde{\mathscr{P}}_{1} \cup\left(\widetilde{\mathscr{P}}_{2} \cap \widetilde{\mathscr{P}}_{3}\right)$ and $\left(\widetilde{\mathscr{P}}_{1} \cap \widetilde{\mathscr{P}}_{2}\right)$ $\cap \widetilde{\mathscr{P}}_{3}=\widetilde{\mathscr{P}}_{1} \cap\left(\widetilde{\mathscr{P}}_{2} \cap \widetilde{\mathscr{P}}_{3}\right)$

(5) $\left(\widetilde{\mathscr{P}}_{1} \cup \widetilde{\mathscr{P}}_{2}\right) \cap \widetilde{\mathscr{P}}_{2}=\widetilde{\mathscr{P}}_{2}$ and $\left(\widetilde{\mathscr{P}}_{1} \cap \widetilde{\mathscr{P}}_{2}\right) \cup \widetilde{\mathscr{P}}_{2}=\widetilde{\mathscr{P}}_{2}$

Proof. They are trial. We omit them. 
3.2. Score Function of IVPHFEs. To determine the priorities of the alternatives of an interval-valued Pythagorean hesitant fuzzy group decision-making problem, we need the concept of score functions for IVPHFEs. Since an IVPHFE includes several pairs formed by possible Pythagorean membership intervals and possible Pythagorean nonmembership intervals, if we use a method similar to Definition 12, the intervals are represented by the average values of the intervals, which must lose some information because there is no one-to-one correspondence between an interval number and a value.

To facilitate comparison of IVPHFEs, we shall give the following comparison laws.

Definition 15. Let $\widetilde{P}=\left\{\langle\widetilde{\mu}, \widetilde{\nu}\rangle \mid \widetilde{\mu}=\left[\mu^{-}, \mu^{+}\right], \widetilde{\nu}=\left[\nu^{-}, \nu^{+}\right]\right\}$ be an IVPHFE. The score function $S(\tilde{\mathscr{P}})$ is described as

$$
\begin{aligned}
S(\widetilde{\mathscr{P}})= & \frac{1}{2|\widetilde{\mathscr{P}}|} \sum_{\langle\widetilde{\mu}, \widetilde{\nu}\rangle \in \widetilde{\mathscr{P}}}\left(\tilde{\mu}^{2}-\widetilde{\nu}^{2}\right)=\left[\frac { 1 } { 2 | \widetilde { \mathscr { P } } | } \sum _ { \langle \widetilde { \mu } , \widetilde { \nu } \rangle \in \widetilde { \mathscr { P } } } \left(\left(\mu^{-}\right)^{2}\right.\right. \\
& \left.\left.-\left(\nu^{+}\right)^{2}\right), \frac{1}{2|\widetilde{\mathscr{P}}|} \sum_{\langle\widetilde{\mu}, \tilde{\nu}\rangle \in \widetilde{\mathscr{P}}}\left(\left(\mu^{+}\right)^{2}-\left(\nu^{-}\right)^{2}\right)\right] .
\end{aligned}
$$

The accuracy function $H(\widetilde{\mathscr{P}})$ is described as

$$
\begin{aligned}
H(\widetilde{\mathscr{P}})= & \frac{1}{2|\widetilde{\mathscr{P}}|} \sum_{\langle\widetilde{\mu}, \widetilde{\nu}\rangle \in \widetilde{\mathscr{P}}}\left(\tilde{\mu}^{2}+\widetilde{\nu}^{2}\right)=\left[\frac { 1 } { 2 | \widetilde { \mathscr { P } } | } \sum _ { \langle \widetilde { \mu } , \widetilde { \nu } \rangle \in \widetilde { \mathscr { P } } } \left(\left(\mu^{-}\right)^{2}\right.\right. \\
& \left.\left.+\left(\nu^{-}\right)^{2}\right), \frac{1}{2|\widetilde{\mathscr{P}}|} \sum_{\langle\widetilde{\mu}, \widetilde{\nu}\rangle \in \widetilde{\mathscr{P}}}\left(\left(\mu^{+}\right)^{2}+\left(\nu^{+}\right)^{2}\right)\right] .
\end{aligned}
$$

The idea of the above concepts originated from Definition 8 and used the definition of interval arithmetic in Definition 6. The score function of an IVPHFE is the mean of the difference between possible Pythagorean membership intervals and possible Pythagorean nonmembership intervals; also, the accuracy function reflects the overall accuracy degree of an IVPHFE. For keeping fuzzy information as much as possible, both the two functions are represented by interval values. For an IVPHFE $\widetilde{\mathscr{P}}$, the score function $S(\widetilde{\mathscr{P}}) \in[-1,1]$ and the accuracy function $H(\widetilde{\mathscr{P}}) \in[0,1]$ hold obviously. Based on Definition 7, we have the following definition.

Definition 16. Let $\widetilde{P}_{1}$ and $\widetilde{\mathscr{P}}_{2}$ be two IVPHFEs.

(1) If $P\left(S\left(\widetilde{\mathscr{P}}_{1}\right)>S\left(\widetilde{\mathscr{P}}_{2}\right)\right)<0.5$, then we say $\widetilde{\mathscr{P}}_{1} \prec \widetilde{\mathscr{P}}_{2}$

(2) If $P\left(S\left(\widetilde{\mathscr{P}}_{1}\right)>S\left(\widetilde{\mathscr{P}}_{2}\right)\right)=0.5$, then

(2.1) If $P\left(H\left(\widetilde{\mathscr{P}}_{1}\right)>H\left(\widetilde{\mathscr{P}}_{2}\right)\right)<0.5$, we say $\widetilde{\mathscr{P}}_{1}<\widetilde{\mathscr{P}}_{2}$

(2.2) If $P\left(H\left(\widetilde{\mathscr{P}}_{1}\right)>H\left(\widetilde{\mathscr{P}}_{2}\right)\right)=0.5$, we say $\widetilde{\mathscr{P}}_{1}=\widetilde{\mathscr{P}}_{2}$

Proposition 4. Let $\widetilde{\mathscr{P}}_{1}=\left\{\left\langle\widetilde{\mu}_{1 j}, \widetilde{\nu}_{1 j}\right\rangle \mid \widetilde{\mu}_{1 j}=\left[\mu_{1 j}^{-}, \mu_{1 j}^{+}\right], \widetilde{\nu}_{1 j}=\right.$ $\left.\left[v_{1 j}^{-}, v_{1 j}^{+}\right], j=1,2, \cdots, m\right\}$ and $\mathscr{P}_{2}=\left\{\left\langle\widetilde{\mu}_{2 j}, \widetilde{v}_{2 j}\right\rangle \mid \tilde{\mu}_{2 j}=\left[\mu_{2 j}^{-}\right.\right.$, $\left.\left.\mu_{2 j}^{+}\right], \widetilde{v}_{2 j}=\left[v_{2 j}^{-}, v_{2 j}^{+}\right], j=1,2, \cdots, m\right\}$ be two IVPHFEs. If for any $j=1, \cdots, m, \mu_{1 j}^{-} \leq \mu_{2 j}^{-}, \mu_{1 j}^{+} \leq \mu_{2 j}^{+}, v_{1 j}^{-} \geq v_{2 j}^{-}$, and $v_{1 j}^{+} \geq v_{2 j}^{+}$, then $\widetilde{P}_{1} \leqslant \widetilde{\mathscr{P}}_{2}$.

Proof. Based on Definition 15, we have

$$
\begin{aligned}
& S\left(\widetilde{\mathscr{P}}_{1}\right)=\frac{1}{2 m} \sum_{j=1}^{m}\left[\left(\mu_{1 j}^{-}\right)^{2}-\left(v_{1 j}^{+}\right)^{2},\left(\mu_{1 j}^{+}\right)^{2}-\left(v_{1 j}^{-}\right)^{2}\right], \\
& S\left(\widetilde{\mathscr{P}}_{2}\right)=\frac{1}{2 m} \sum_{j=1}^{m}\left[\left(\mu_{2 j}^{-}\right)^{2}-\left(v_{2 j}^{+}\right)^{2},\left(\mu_{2 j}^{+}\right)^{2}-\left(v_{2 j}^{-}\right)^{2}\right] .
\end{aligned}
$$

Suppose

$$
L \triangleq \frac{\sum_{j=1}^{m}\left(\left(\mu_{2 j}^{+}\right)^{2}-\left(v_{2 j}^{-}\right)^{2}+\left(v_{1 j}^{+}\right)^{2}-\left(\mu_{1 j}^{-}\right)^{2}\right)}{\sum_{j=1}^{m}\left(\left(\mu_{1 j}^{+}\right)^{2}-\left(v_{1 j}^{-}\right)^{2}+\left(v_{1 j}^{+}\right)^{2}-\left(\mu_{1 j}^{-}\right)^{2}+\left(\mu_{2 j}^{+}\right)^{2}-\left(v_{2 j}^{-}\right)^{2}+\left(v_{2 j}^{+}\right)^{2}-\left(\mu_{2 j}^{-}\right)^{2}\right)} .
$$

Hence, we obtain $\quad P\left(S\left(\widetilde{\mathscr{P}}_{1}\right)>S\left(\widetilde{\mathscr{T}}_{2}\right)\right)=\max$ $\{1-\max \{L, 0\}, 0\}$ by Definition 7 .

For any $j=1, \cdots, m, \mu_{1 j}^{-} \leq \mu_{2 j}^{-}, \mu_{1 j}^{+} \leq \mu_{2 j}^{+}, v_{1 j}^{-} \geq v_{2 j}^{-}$, and $v_{1 j}^{+} \geq v_{2 j}^{+},\left(\mu_{2 j}^{+}\right)^{2}-\left(\mu_{1 j}^{-}\right)^{2}+\left(v_{1 j}^{+}\right)^{2}-\left(v_{2 j}^{-}\right)^{2} \geq\left(\mu_{1 j}^{+}\right)^{2}-\left(\mu_{2 j}^{-}\right)^{2}+$ $\left(v_{2 j}^{+}\right)^{2}-\left(\nu_{1 j}^{-}\right)^{2} \geq 0$; then, $0.5 \leq L \leq 1$. So, $0 \leq 1-\max \{L, 0\}=$ $1-L \leq 0.5$. 'Hence, $P\left(S\left(\widetilde{\mathscr{P}}_{1}\right)>S\left(\widetilde{\mathscr{P}}_{2}\right)\right)=1-L \leq 0.5$. That means $\widetilde{\mathscr{P}}_{1} \leqslant \widetilde{\mathscr{P}}_{2}$ holds.

\section{Aggregation Operators for Interval-Valued Pythagorean Hesitant Fuzzy Information}

In multiattribute decision-making problems, the selection of aggregation operators is a basis problem, which is also important in the interval-valued Pythagorean hesitant fuzzy environment. Considering an IVPHFE is regarded as the extension of an IVHFE, IVPFE, or PHFE, we propose a series of aggregation operators for intervalvalued Pythagorean hesitant fuzzy information in this section based on the discussion of aggregation operators in $[27,45,50,51]$ and deduce some desirable properties.

4.1. The IVPHFWA, IVPHFWG, GIVPHFWA, and GIVPHFWG Operators

Definition 17. Let $\widetilde{\mathscr{P}}_{i}(i=1,2, \cdots, n)$ be a collection of some IVPHFEs and $\omega=\left(\omega_{1}, \omega_{2}, \cdots, \omega_{n}\right)^{T}$ be the weight vector of $\widetilde{\mathscr{P}}_{i}(i=1,2, \cdots, n)$, with $\omega_{i} \in[0,1], \sum_{i=1}^{n} \omega_{i}=1$, and $\lambda>0$. 
(1) An interval-valued Pythagorean hesitant fuzzy weighted averaging (IVPHFWA) operator can be seen a map IVPHFWA: $\Omega^{n} \longrightarrow \Omega$, such that

$\operatorname{IVPHFWA}\left(\widetilde{\mathscr{P}}_{1}, \cdots, \widetilde{\mathscr{P}}_{n}\right)=\omega_{1} \widetilde{\mathscr{P}}_{1} \oplus \cdots \oplus \omega_{n} \widetilde{\mathscr{P}}_{n}$

$$
\begin{aligned}
= & \left\{\left\langle\sqrt{\left\langle 1-\prod_{i=1}^{n}\left(1-\left(\mu_{i}^{-}\right)^{2}\right)^{\omega_{i}},\right.}\right.\right. \\
& \sqrt{\left.1-\prod_{i=1}^{n}\left(1-\left(\mu_{i}^{+}\right)^{2}\right)^{\omega_{i}}\right],} \\
& {\left.\left[\prod_{i=1}^{n}\left(\nu_{i}^{-}\right)^{\omega_{i}}, \prod_{i=1}^{n}\left(\nu_{i}^{+}\right)^{\omega_{i}}\right]\right\rangle } \\
& \left.\mid\left\langle\tilde{\mu}_{i}, \widetilde{v}\right\rangle_{i} \in \widetilde{\mathscr{P}}_{i}, i=1, \cdots, n\right\} .
\end{aligned}
$$

(2) An interval-valued Pythagorean hesitant fuzzy weighted geometric (IVPHFWG) operator can be seen a map IVPHFWG: $\Omega^{n} \longrightarrow \Omega$, such that

$$
\begin{aligned}
\operatorname{IVPHFWG}\left(\widetilde{\mathscr{P}}_{1}, \ldots, \widetilde{\mathscr{P}}_{n}\right)= & \widetilde{\mathscr{P}}_{1}^{\omega_{1}} \otimes \cdots \otimes \widetilde{\mathscr{P}}_{n}^{\omega_{n}} \\
= & \left\{\left\langle\left[\prod_{i=1}^{n}\left(\mu_{i}^{-}\right)^{\omega_{i}}, \prod_{i=1}^{n}\left(\mu_{i}^{+}\right)^{\omega_{i}}\right],\right.\right. \\
& \cdot\left[\sqrt{1-\prod_{i=1}^{n}\left(1-\left(v_{i}^{-}\right)^{2}\right)^{\omega_{i}},}\right. \\
& \cdot \sqrt{\left.\left.1-\prod_{i=1}^{n}\left(1-\left(v_{i}^{+}\right)^{2}\right)^{\omega_{i}}\right]\right\rangle \mid\left\langle\widetilde{\mu}_{i}, \widetilde{v}_{i}\right\rangle} \\
& \left.\in \widetilde{\mathscr{P}}_{i}, i=1, \cdots, n\right\} .
\end{aligned}
$$

(3) A generalized interval-valued Pythagorean hesitant fuzzy weighted averaging (GIVPHFWA) operator can be seen a map GIVPHFWA: $\Omega^{n} \longrightarrow \Omega$, which satisfies

$$
\begin{aligned}
& \operatorname{GIVPHFWA}_{\lambda}\left(\widetilde{\mathscr{P}}_{1}, \cdots, \widetilde{\mathscr{P}}_{n}\right)=\left(\omega_{1} \widetilde{\mathscr{P}}_{1}^{\lambda} \oplus \cdots \oplus \omega_{n} \widetilde{\mathscr{P}}_{n}^{\lambda}\right)^{1 / \lambda} \\
& \begin{aligned}
= & \left\langle\left[\sqrt{\left(1-\prod_{i=1}^{n}\left(1-\left(\mu_{i}^{-}\right)^{2 \lambda}\right)^{\omega_{i}}\right)^{1 / \lambda}}, \sqrt{\left(1-\prod_{i=1}^{n}\left(1-\left(\mu_{i}^{+}\right)^{2 \lambda}\right)^{\omega_{i}}\right)^{1 / \lambda}}\right],\right. \\
& \left.\cdot\left[\sqrt{1-\left(1-\prod_{i=1}^{n}\left(1-\left(1-\left(v_{i}^{-}\right)^{2}\right)^{\lambda}\right)^{\omega_{i}}\right)^{1 / \lambda}}, \sqrt{1-\left(1-\prod_{i=1}^{n}\left(1-\left(1-\left(v_{i}^{+}\right)^{2}\right)^{\lambda}\right)^{\omega_{i}}\right)^{1 / \lambda}}\right]\right\rangle \mid\left\langle\widetilde{\mu}_{i}, \widetilde{v}_{i}\right\rangle
\end{aligned} \\
& \left.\in \widetilde{\mathscr{P}}_{i}, i=1, \cdots, n\right\} \text {, }
\end{aligned}
$$

where $\lambda>0$.

(4) A generalized interval-valued Pythagorean hesitant fuzzy weighted geometric (GIVPHFWG) operator can be seen a map GIVPHFWG: $\Omega^{n} \longrightarrow \Omega$, which satisfies

$$
\begin{aligned}
\operatorname{GIVPHFWG}_{\lambda}\left(\widetilde{\mathscr{P}}_{1}, \cdots, \widetilde{\mathscr{P}}_{n}\right)= & \frac{1}{\lambda}\left(\left(\lambda \widetilde{\mathscr{P}}_{1}\right)^{\omega_{1}} \otimes \cdots \otimes\left(\lambda \widetilde{\mathscr{P}}_{n}\right)^{\omega_{n}}\right) \\
= & \left\langle\left\langle\sqrt{1-\left(1-\prod_{i=1}^{n}\left(1-\left(1-\left(\mu_{i}^{-}\right)^{2}\right)^{\lambda}\right)^{\omega_{i}}\right)^{1 / \lambda}}, \sqrt{1-\left(1-\prod_{i=1}^{n}\left(1-\left(1-\left(\mu_{i}^{+}\right)^{2}\right)^{\lambda}\right)^{\omega_{i}}\right)^{1 / \lambda}},\right.\right. \\
& \left.\left.\cdot\left[\sqrt{\left(1-\prod_{i=1}^{n}\left(1-\left(v_{i}^{-}\right)^{2 \lambda}\right)^{\omega_{i}}\right)^{1 / \lambda}}, \sqrt{\left(1-\prod_{i=1}^{n}\left(1-\left(v_{i}^{+}\right)^{2 \lambda}\right)^{\omega_{i}}\right)^{1 / \lambda}}\right]\right\rangle, \mid\left\langle\widetilde{\mu}_{i}, \widetilde{v}_{i}\right\rangle \in \widetilde{\mathscr{P}}_{i}, i=1, \cdots, n\right\},
\end{aligned}
$$

where $\lambda>0$. If $\lambda=1$, then the GIVPHFWA and GIVPHFWG operators reduce to the IVPHFWA and IVPHFWG operators, respectively.
Example 1. Suppose $\widetilde{\mathscr{P}}_{1}=\{\langle[0.3,0.5],[0.2,0.4]\rangle\}$, 


$$
\begin{aligned}
& \widetilde{\mathscr{P}}_{2}=\{\langle[0.1,0.4],[0.3,0.6]\rangle,\langle[0.1,0.4],[0.5,0.7]\rangle\}, \\
& \widetilde{\mathscr{P}}_{3}=\{\langle[0.3,0.4],[0.2,0.6]\rangle,\langle[0.1,0.3],[0.2,0.6]\rangle,\langle[0.4,0.7],[0.2,0.6]\rangle\},
\end{aligned}
$$

are three IVPHFEs, and their weight vector is Then, by Definition 17, we have $\omega=(0.15,0.30,0.55)^{T}$.

$$
\begin{aligned}
& \operatorname{IVPHFWA}\left(\widetilde{\mathscr{P}}_{1}, \widetilde{\mathscr{P}}_{2}, \widetilde{\mathscr{P}}_{3}\right)=\omega_{1} \widetilde{\mathscr{P}}_{1} \oplus \omega_{1} \widetilde{\mathscr{P}}_{2} \oplus \omega_{n} \widetilde{\mathscr{P}}_{3} \\
& =\left\{\left\langle\sqrt{1-\prod_{i=1}^{3}\left(1-\left(\mu_{i}^{-}\right)^{2}\right)^{\omega_{i}}}, \sqrt{1-\prod_{i=1}^{3}\left(1-\left(\mu_{i}^{+}\right)^{2}\right)^{\omega_{i}}}\right],\right. \\
& \left.\left.\cdot\left[\prod_{i=1}^{3}\left(v_{i}^{-}\right)^{\omega_{i}}, \prod_{i=1}^{3}\left(v_{i}^{+}\right)^{\omega_{i}}\right]\right\rangle \mid\left\langle\widetilde{\mu}_{i}, \widetilde{v}_{i}\right\rangle \in \widetilde{\mathscr{P}}_{i}, i=1,2,3\right\} \\
& =\{\langle[0.2583,0.4173],[0.2259,0.5646]\rangle,\langle[0.1498,0.3701],[0.2259,0.5646]\rangle \text {, } \\
& \cdot\langle[0.3270,0.6102],[0.2259,0.5646]\rangle,\langle[0.2583,0.4173],[0.2633,0.5913]\rangle, \\
& \cdot\langle[0.1498,0.3701],[0.2633,0.5913]\rangle,\langle[0.3270,0.6102],[0.2633,0.5913]\rangle\} ;
\end{aligned}
$$

$\operatorname{IVPHFWG}\left(\widetilde{\mathscr{P}}_{1}, \widetilde{\mathfrak{P}}_{2}, \widetilde{\mathfrak{P}}_{3}\right)=\widetilde{\mathscr{P}}_{1}^{\omega_{1}} \otimes \widetilde{\mathscr{P}}_{3}^{\omega_{2}} \otimes \widetilde{\mathscr{P}}_{3}^{\omega_{3}}$

$$
\begin{aligned}
= & \left\{\left\langle\left[\prod_{i=1}^{3}\left(\mu_{i}^{-}\right)^{\omega_{i}}, \prod_{i=1}^{3}\left(\mu_{i}^{+}\right)^{\omega_{i}}\right],\left[\sqrt{1-\prod_{i=1}^{3}\left(1-\left(v_{i}^{-}\right)^{2}\right)^{\omega_{i}}}, \sqrt{\left.1-\prod_{i=1}^{3}\left(1-\left(v_{i}^{+}\right)^{2}\right)^{\omega_{i}}\right]}\right\rangle\right.\right. \\
& \left.\cdot \mid\left\langle\widetilde{\mu}_{i}, \widetilde{v}_{i}\right\rangle \in \widetilde{\mathscr{P}}_{i}, i=1,2,3\right\} .
\end{aligned}
$$

$=\{\langle[0.2158,0.4136],[0.2351,0.5774]\rangle,\langle[0.1179,0.3531],[0.2351,0.5774]\rangle$,

$\cdot\langle[0.2528,0.5627],[0.2351,0.5774]\rangle,\langle[0.2158,0.4137],[0.3294,0.6142]\rangle,\langle[0.1179,0.3531]$, $\cdot[0.3294,0.6142]\rangle,\langle[0.2528,0.5627],[0.3294,0.6142]\rangle\} ;$

$\operatorname{GIVPHFWA}_{3}\left(\widetilde{\mathscr{P}}_{1}, \widetilde{\mathscr{P}}_{2}, \widetilde{\mathscr{P}}_{3}\right)=\left(\omega_{1} \widetilde{\mathscr{P}}_{1}^{3} \oplus \omega_{2} \widetilde{\mathscr{P}}_{2}^{3} \oplus \omega_{3} \widetilde{\mathscr{P}}_{3}^{3}\right)^{1 / 3}$

$$
\begin{aligned}
& =\left\{\left\langle\sqrt{\left(1-\prod_{i=1}^{3}\left(1-\left(\mu_{i}^{-}\right)^{2 \times 3}\right)^{\omega_{i}}\right)^{1 / 3}}, \sqrt{\left(1-\prod_{i=1}^{3}\left(1-\left(\mu_{i}^{+}\right)^{2 \times 3}\right)^{\omega_{i}}\right)^{1 / 3}}\right],\right. \\
& \left.\left..\left[\sqrt{1-\left(1-\prod_{i=1}^{3}\left(1-\left(1-\left(v_{i}^{-}\right)^{2}\right)^{3}\right)^{\omega_{i}}\right)^{1 / 3}}, \sqrt{1-\left(1-\prod_{i=1}^{3}\left(1-\left(1-\left(v_{i}^{+}\right)^{2}\right)^{3}\right)^{\omega_{i}}\right)^{1 / 3}}\right]\right\rangle, \mid\left\langle\widetilde{\mu}_{i}, \widetilde{v}_{i}\right\rangle \in \widetilde{\mathscr{P}}_{i}, i=1,2,3\right\},
\end{aligned}
$$

$=\{\langle[0.2827,0.4243],[0.2254,0.5589]\rangle,\langle[0.2190,0.3982],[0.2254,0.5589]\rangle,\langle[0.3650,0.6418]$,

$\cdot[0.2254,0.5589]\rangle,\langle[0.2827,0.4243],[0.2582,0.5805]\rangle,\langle[0.2190,0.3982],[0.2582,0.5805]\rangle,\langle[0.3650,0.6418]$, $\cdot[0.2582,0.5805]\rangle\}$

$\operatorname{GIVPHFWG}\left(\widetilde{\mathscr{P}}_{1}, \widetilde{\mathscr{P}}_{2}, \widetilde{\mathscr{P}}_{3}\right)=\frac{1}{3}\left(\left(3 \widetilde{\mathscr{P}}_{1}\right)^{\omega_{1}} \otimes\left(3 \widetilde{\mathscr{P}}_{2}\right)^{\omega_{2}} \otimes\left(3 \widetilde{\mathfrak{P}}_{3}\right)^{\omega_{3}}\right)$

$$
\begin{gathered}
=\left\{\left\langle\left[\sqrt{1-\left(1-\prod_{i=1}^{3}\left(1-\left(1-\left(\mu_{i}^{-}\right)^{2}\right)^{3}\right)^{\omega_{i}}\right)^{1 / 3}}, \sqrt{1-\left(1-\prod_{i=1}^{3}\left(1-\left(1-\left(\mu_{i}^{+}\right)^{2}\right)^{3}\right)^{\omega_{i}}\right)^{1 / 3}}\right],\right.\right. \\
\left.\left..\left[\sqrt{\left(1-\prod_{i=1}^{3}\left(1-\left(v_{i}^{-}\right)^{2 \times 3}\right)^{\omega_{i}}\right)^{1 / 3}}, \sqrt{\left(1-\prod_{i=1}^{3}\left(1-\left(\nu_{i}^{+}\right)^{2 \times 3}\right)^{\omega_{i}}\right)^{1 / 3}}\right]\right\rangle, \mid\left\langle\widetilde{\mu}_{i}, \widetilde{\nu}_{i}\right\rangle \in \widetilde{\mathscr{P}}_{i}, i=1,2,3\right\},
\end{gathered}
$$

$=\{\langle[0.2135,0.4129],[0.2532,0.5858]\rangle,\langle[0.1174,0.3508],[0.2532,0.5858]\rangle,\langle[0.2471,0.5437],[0.2532,0.5858]\rangle$,

$\cdot\langle[0.2135,0.4129],[0.4101,0.6298]\rangle,\langle[0.1174,0.3508],[0.4101,0.6298]\rangle,\langle[0.2471,0.5437],[0.4101,0.6298]\rangle\}$. 
Based on Proposition 1, we know the above four aggregation operators are all IVPHFEs. Also, we can obtain that the score values are

$$
\begin{aligned}
& S\left(\operatorname{IVPHFWA}\left(\widetilde{\mathscr{P}}_{1}, \widetilde{\mathscr{P}}_{2}, \widetilde{\mathscr{P}}_{3}\right)\right)=[-0.1344,0.0838] \text {; } \\
& S\left(\operatorname{IVPHFWG}\left(\widetilde{\mathscr{P}}_{1}, \widetilde{\mathscr{P}}_{2}, \widetilde{\mathscr{P}}_{3}\right)\right)=[-0.1569,0.0611] \text {; } \\
& S\left(\operatorname{GIVPHFWA}_{3}\left(\widetilde{\mathscr{P}}_{1}, \widetilde{\mathscr{P}}_{2}, \widetilde{\mathscr{P}}_{3}\right)\right)=[-0.1188,0.0957] ; \\
& S\left(\operatorname{GIVPHFWG}_{3}\left(\widetilde{\mathscr{P}}_{1}, \widetilde{\mathscr{P}}_{2}, \widetilde{\mathscr{P}}_{3}\right)\right)=[-0.1649,0.0401] ; \\
& \text { So, } P\left(\operatorname{SIVPHFWG}\left(\widetilde{\mathscr{P}}_{1}, \widetilde{\mathscr{P}}_{2}, \widetilde{\mathscr{P}}_{3}\right) \leq S\left(\operatorname{IVPHFWA}\left(\widetilde{\mathscr{P}}_{1}, \widetilde{\mathscr{P}}_{2}, \widetilde{\mathscr{P}}_{3}\right)\right)\right)=0.5518 \text {; }
\end{aligned}
$$

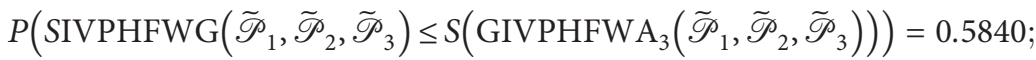

$$
\begin{aligned}
& P\left(\operatorname{SGIVPHFWG}{ }_{3}\left(\widetilde{\mathscr{P}}_{1}, \widetilde{\mathscr{P}}_{2}, \widetilde{P}_{3}\right) \leq S\left(\operatorname{IVPHFWA}\left(\widetilde{\mathscr{P}}_{1}, \widetilde{\mathscr{P}}_{2}, \widetilde{\mathscr{P}}_{3}\right)\right)\right)=0.5876 \text {; } \\
& \text { Hence, } \operatorname{IVPHFWG}\left(\widetilde{\mathscr{P}}_{1}, \widetilde{\mathscr{P}}_{2}, \widetilde{\mathscr{P}}_{3}\right) \leqslant \operatorname{IVPhHFA}\left(\widetilde{\mathscr{P}}_{1}, \widetilde{\mathscr{P}}_{2}, \widetilde{\mathscr{P}}_{3}\right) \text {; } \\
& \operatorname{IVPHFWG}\left(\widetilde{\mathscr{P}}_{1}, \widetilde{\mathscr{P}}_{2}, \widetilde{\mathscr{P}}_{3}\right) \leqslant \operatorname{GIVPHFWA}\left(\widetilde{\mathscr{P}}_{1}, \widetilde{\mathscr{P}}_{2}, \widetilde{\mathscr{P}}_{3}\right) \text {; } \\
& \operatorname{GIVPHFWG}{ }_{3}\left(\widetilde{\mathscr{P}}_{1}, \widetilde{\mathscr{P}}_{2}, \widetilde{\mathfrak{P}}_{3}\right) \leqslant \operatorname{IVPHFWA}\left(\widetilde{\mathscr{P}}_{1}, \widetilde{\mathscr{P}}_{2}, \widetilde{\mathscr{P}}_{3}\right) \text {. }
\end{aligned}
$$

In fact, we have the following.

Lemma 1 (see [52]). Let $x_{i}>0, \omega_{i}>0(i=1,2, \cdots, n)$, and $\sum_{i=1}^{n} \omega_{i}=1$, then

$$
\prod_{i=1}^{n} x_{i}^{\omega_{i}} \leq \sum_{i=1}^{n} \omega_{i} x_{i}
$$

with equality if and only if $x_{1}=x_{2}=\cdots=x_{n}$.
Proposition 5. Let $\widetilde{\mathscr{P}}_{i}(i=1,2, \cdots, n)$ be a collection of IVPHFEs, $\omega_{i}>0$, and $\sum_{i=1}^{n} \omega_{i}=1$, then

$\operatorname{IVPHFWG}\left(\widetilde{\mathscr{P}}_{1}, \widetilde{\mathscr{P}}_{2}, \cdots, \widetilde{\mathscr{P}}_{n}\right) \leqslant \operatorname{IVPHFWA}\left(\widetilde{\mathscr{P}}_{1}, \widetilde{\mathscr{P}}_{2}, \cdots, \widetilde{\mathscr{P}}_{n}\right)$,

with equality if and only if $\widetilde{\mathscr{P}}_{1}=\widetilde{\mathscr{P}}_{2}=\cdots=\widetilde{\mathscr{P}}_{n}$.

Proof. Suppose $\left\langle\left[\mu_{i}^{-}, \mu_{i}^{+}\right],\left[\nu_{i}^{-}, \nu_{i}^{+}\right]\right\rangle \in \widetilde{\mathscr{P}}_{i}$, for any $i$. Based on Lemma 1, we have

$$
\prod_{i=1}^{n}\left(\left(\mu_{i}^{-}\right)^{2}\right)^{\omega_{i}} \leq \sum_{i=1}^{n} \omega_{i}\left(\mu_{i}^{-}\right)^{2}=1-\sum_{i=1}^{n} \omega_{i}\left(1-\left(\mu_{i}^{-}\right)^{2}\right) \leq 1-\prod_{i=1}^{n}\left(1-\left(\mu_{i}^{-}\right)^{2}\right)^{\omega_{i}} .
$$

So,

$$
\prod_{i=1}^{n}\left(\mu_{i}^{-}\right)^{\omega_{i}}=\sqrt{\prod_{i=1}^{n}\left(\left(\mu_{i}^{-}\right)^{2}\right)^{\omega_{i}}} \leq
$$

$\operatorname{IVPHFWG}\left(\widetilde{\mathscr{P}}_{1}, \widetilde{\mathscr{P}}_{2}, \cdots, \widetilde{\mathscr{P}}_{n}\right) \leqslant \operatorname{IVPHFWA}\left(\widetilde{\mathscr{P}}_{1}, \widetilde{\mathscr{P}}_{2}, \cdots, \widetilde{\mathscr{P}}_{n}\right)$.

$\sqrt{1-\prod_{i=1}^{n}\left(1-\left(\mu_{i}^{-}\right)^{2}\right)^{\omega_{i}}}$. Similarly, we have

$$
\begin{aligned}
& \prod_{i=1}^{n}\left(\mu_{i}^{+}\right)^{\omega_{i}} \leq \sqrt{1-\prod_{i=1}^{n}\left(1-\left(\mu_{i}^{+}\right)^{2}\right)^{\omega_{i}},} \\
& \prod_{i=1}^{n}\left(\nu_{i}^{-}\right)^{\omega_{i}} \leq \sqrt{1-\prod_{i=1}^{n}\left(1-\left(v_{i}^{-}\right)^{2}\right)^{\omega_{i}}}, \\
& \prod_{i=1}^{n}\left(v_{i}^{+}\right)^{\omega_{i}} \leq \sqrt{1-\prod_{i=1}^{n}\left(1-\left(v_{i}^{+}\right)^{2}\right)^{\omega_{i}} .}
\end{aligned}
$$

By Definition 17 and Proposition $P\left(S\left(\widetilde{\mathscr{P}}_{1}^{\omega_{1}} \otimes \cdots \otimes \widetilde{\mathscr{P}}_{n}^{\omega_{n}}\right) \geq S\left(\omega_{1} \widetilde{\mathscr{P}}_{1} \oplus \cdots \oplus \omega_{n} \widetilde{\mathscr{P}}_{n}\right)\right) \leq 0.5$ holds, which means

Proposition 6. Let $\widetilde{\mathscr{P}}_{i}(i=1,2, \cdots, n)$ be a collection of IVPHFEs, $\lambda>0, \omega_{i}>0$, and $\sum_{i=1}^{n} \omega_{i}=1$, then $\operatorname{IVPHFWG}\left(\widetilde{\mathscr{P}}_{1}, \widetilde{\mathfrak{P}}_{2}, \cdots, \widetilde{\mathscr{P}}_{n}\right) \leqslant \operatorname{GIVPHFWA}\left(\widetilde{\mathscr{P}}_{1}, \widetilde{\mathscr{P}}_{2}, \cdots, \widetilde{P}_{n}\right)$, $\operatorname{GIVPHFWG}_{\lambda}\left(\widetilde{\mathscr{P}}_{1}, \widetilde{\mathscr{P}}_{2}, \cdots, \widetilde{\mathscr{P}}_{n}\right) \leqslant \operatorname{IVPHFWA}\left(\widetilde{\mathscr{P}}_{1}, \widetilde{\mathscr{P}}_{2}, \cdots, \widetilde{\mathscr{P}}_{n}\right)$.

Proof. Suppose $\left\langle\left[\mu_{i}^{-}, \mu_{i}^{+}\right],\left[v_{i}^{-}, v_{i}^{+}\right]\right\rangle \in \widetilde{\mathscr{P}}_{i}$, for any $i$. Since $\prod_{i=1}^{n}\left(\left(\mu_{i}^{-}\right)^{2}\right)^{\omega_{i}}=\left(\prod_{i=1}^{n}\left(\left(\mu_{i}^{-}\right)^{2 \lambda}\right)^{\omega_{i}}\right)^{1 / \lambda} \leq\left(\sum_{i=1}^{n} \omega_{i}\left(\mu_{i}^{-}\right)^{2 \lambda}\right)^{1 / \lambda}=$ $\left(1-\sum_{i=1}^{n} \omega_{i}\left(1-\left(\mu_{i}^{-}\right)^{2 \lambda}\right)\right)^{1 / \lambda} \leq 1-\left(\prod_{i=1}^{n}\left(1-\left(\mu_{i}^{-}\right)^{2 \lambda}\right)^{\omega_{i}}\right)^{1 / \lambda}$, we have $\quad \prod_{i=1}^{n}\left(\mu_{i}^{-}\right)^{\omega_{i}}=\sqrt{\prod_{i=1}^{n}\left(\left(\mu_{i}^{-}\right)^{2}\right)^{\omega_{i}}} \leq$ 4, $\quad \sqrt{\left(1-\prod_{i=1}^{n}\left(1-\left(\mu_{i}^{-}\right)^{2 \lambda}\right)^{\omega_{i}}\right)^{1 / \lambda}}$. Similarly, $\quad \prod_{i=1}^{n}\left(\mu_{i}^{+}\right)^{\omega_{i}}=\sqrt{\prod_{i=1}^{n}\left(\left(\mu_{i}^{+}\right)^{2}\right)^{\omega_{i}}} \leq$ $\sqrt{\left(1-\prod_{i=1}^{n}\left(1-\left(\mu_{i}^{+}\right)^{2 \lambda}\right)^{\omega_{i}}\right)^{1 / \lambda}}$ holds. 
Also,

$$
\begin{aligned}
\sqrt{1-\left(1-\prod_{i=1}^{n}\left(1-\left(1-\left(v_{i}^{-}\right)^{2}\right)^{\lambda}\right)^{\omega_{i}}\right)^{1 / \lambda}} & \leq \sqrt{1-\left(1-\sum_{i=1}^{n} \omega_{i}\left(1-\left(1-\left(v_{i}^{-}\right)^{2}\right)^{\lambda}\right)\right)^{1 / \lambda}} \\
& =\sqrt{1-\left(\sum_{i=1}^{n} \omega_{i}\left(1-\left(v_{i}^{-}\right)^{2}\right)^{\lambda}\right)^{1 / \lambda}} \leq \sqrt{1-\left(\prod_{i=1}^{n}\left(\left(1-\left(v_{i}^{-}\right)^{2}\right)^{\lambda}\right)^{\omega_{i}}\right)^{1 / \lambda}} \\
& =\sqrt{1-\prod_{i=1}^{n}\left(1-\left(v_{i}^{-}\right)^{2}\right)^{\omega_{i}}} .
\end{aligned}
$$

Similarly, $\quad \sqrt{1-\left(1-\prod_{i=1}^{n}\left(1-\left(1-\left(v_{i}^{+}\right)^{2}\right)^{\lambda}\right)^{\omega_{i}}\right)^{1 / \lambda}} \leq$ $\sqrt{1-\prod_{i=1}^{n}\left(1-\left(v_{i}^{+}\right)^{2}\right)^{\omega_{i}}}$ holds.

All imply that $\operatorname{IVPHFWG}\left(\widetilde{\mathscr{P}}_{1}, \ldots, \widetilde{\mathscr{P}}_{n}\right) \leqslant$ $\operatorname{GIVPHFWA}_{\lambda}\left(\widetilde{\mathscr{P}}_{1}, \ldots, \widetilde{\mathscr{P}}_{n}\right)$.

In the same way, we have $\operatorname{GIVPHFWG}_{\lambda}\left(\widetilde{\mathscr{P}}_{1}, \ldots, \widetilde{\mathscr{P}}_{n}\right) \preccurlyeq$ $\operatorname{IVPHFWA}\left(\widetilde{\mathscr{P}}_{1}, \ldots, \widetilde{\mathcal{P}}_{n}\right)$ and complete the proof of Proposition 6.
Proposition 4.2 shows no matter how the parameter $\lambda(\lambda>0)$ changes, the values obtained by IVPHFWG operators are not bigger than the ones obtained by GIVPHFWA operators and the values obtained by GIVPHFWG operators are not bigger than the ones obtained by IVPHFWA operators.

Proposition 7. Let $\widetilde{\mathfrak{P}}_{i}(i=1,2, \cdots, n)$ be a collection of IVPHFEs, $\lambda_{2} \geq \lambda_{1}>0, \omega_{i}>0$, and $\sum_{i=1}^{n} \omega_{i}=1$, then

$$
\begin{array}{r}
\operatorname{GIVPHFWA}_{\lambda_{1}}\left(\widetilde{\mathscr{P}}_{1}, \widetilde{\mathscr{P}}_{2}, \ldots, \widetilde{\mathscr{P}}_{n}\right) \leqslant \operatorname{GIVPHFWA}_{\lambda_{2}}\left(\widetilde{\mathscr{P}}_{1}, \widetilde{\mathscr{P}}_{2}, \ldots, \widetilde{\mathscr{P}}_{n}\right), \\
\operatorname{GIVPHFWG}_{\lambda_{1}}\left(\widetilde{\mathscr{P}}_{1}, \widetilde{\mathscr{P}}_{2}, \ldots, \widetilde{\mathscr{P}}_{n}\right) \geqslant \operatorname{GIVPHFWG}_{\lambda_{2}}\left(\widetilde{\mathscr{P}}_{2}, \widetilde{\mathscr{P}}_{2}, \ldots, \widetilde{\mathscr{P}}_{n}\right) .
\end{array}
$$

Proof. According to Theorem 3.8 in [31], we have

$$
\begin{gathered}
\sqrt{\left(1-\prod_{i=1}^{n}\left(1-\left(\mu_{i}^{-}\right)^{2 \lambda_{1}}\right)^{\omega_{i}}\right)^{1 / \lambda_{1}}} \leq \sqrt{\left(1-\prod_{i=1}^{n}\left(1-\left(\mu_{i}^{-}\right)^{2 \lambda_{2}}\right)^{\omega_{i}}\right)^{1 / \lambda_{2}}}, \\
\sqrt{\left(1-\prod_{i=1}^{n}\left(1-\left(\mu_{i}^{+}\right)^{2 \lambda_{1}}\right)^{\omega_{i}}\right)^{1 / \lambda_{1}}} \leq \sqrt{\left(1-\prod_{i=1}^{n}\left(1-\left(\mu_{i}^{+}\right)^{2 \lambda_{2}}\right)^{\omega_{i}}\right)^{1 / \lambda_{2}}} .
\end{gathered}
$$

Also, we can conclude that

$$
\sqrt{1-\left(1-\prod_{i=1}^{n}\left(1-\left(1-\left(v_{i}^{-}\right)^{2}\right)^{\lambda_{1}}\right)^{\omega_{i}}\right)^{1 / \lambda_{1}}} \geq \sqrt{1-\left(1-\prod_{i=1}^{n}\left(1-\left(1-\left(v_{i}^{-}\right)^{2}\right)^{\lambda_{2}}\right)^{\omega_{i}}\right)^{1 / \lambda_{2}}} .
$$

Similarly,

$$
\sqrt{1-\left(1-\prod_{i=1}^{n}\left(1-\left(1-\left(v_{i}^{+}\right)^{2}\right)^{\lambda_{1}}\right)^{\omega_{i}}\right)^{1 / \lambda_{1}}} \geq \sqrt{1-\left(1-\prod_{i=1}^{n}\left(1-\left(1-\left(v_{i}^{+}\right)^{2}\right)^{\lambda_{2}}\right)^{\omega_{i}}\right)^{1 / \lambda_{2}}} .
$$


Therefore, by Proposition 4, we have

$$
\begin{gathered}
\operatorname{GIVPHFWA}_{\lambda_{1}}\left(\widetilde{\mathscr{P}}_{1}, \widetilde{\mathscr{P}}_{2}, \ldots, \widetilde{\mathscr{P}}_{n}\right) \preccurlyeq \operatorname{GIVPHFWA}_{\lambda_{2}}\left(\widetilde{\mathscr{P}}_{1}, \widetilde{\mathscr{P}}_{2}, \ldots, \widetilde{\mathscr{P}}_{n}\right), \\
\operatorname{GIVPHFWG}_{\lambda_{1}}\left(\widetilde{\mathscr{P}}_{1}, \widetilde{\mathscr{P}}_{2}, \ldots, \widetilde{\mathscr{P}}_{n}\right) \geqslant \operatorname{GIVPHFWG} \lambda_{\lambda_{2}}\left(\widetilde{\mathscr{P}}_{2}, \widetilde{\mathscr{P}}_{2}, \ldots, \widetilde{\mathscr{P}}_{n}\right) .
\end{gathered}
$$

4.2. The IVPHFOWA, IVPHFOWG, GIVPHFOWA, and GIVPHFOWG Operators

Definition 18. Let $\widetilde{\mathscr{P}}_{i}(i=1,2, \cdots, n)$ be a collection of some IVPHFEs, $\widetilde{\mathscr{P}}_{\sigma(i)}$ be the $i^{\text {th }}$ largest of them, and $\kappa=\left(\kappa_{1}, \kappa_{2}, \cdots, \kappa_{n}\right)^{T}$ be the associated vector such that $\kappa_{i} \in[0,1], \sum_{i=1}^{n} \kappa_{i}=1$, and $\lambda>0$. Then,

(1) An interval-valued Pythagorean hesitant fuzzy ordered weighted averaging (IVPHFOWA) operator can be seen a map IVPHOFWA: $\Omega^{n} \longrightarrow \Omega$, such that

$\operatorname{IVPHFOWA}\left(\widetilde{\mathscr{P}}_{1}, \cdots, \widetilde{\mathscr{P}}_{n}\right)=\kappa_{1} \widetilde{\mathscr{P}}_{\sigma(1)} \oplus \cdots \oplus \kappa_{n} \widetilde{\mathscr{P}}_{\sigma(n)}$

$$
\begin{aligned}
= & \left\langle\left\langle\sqrt{1-\prod_{i=1}^{n}\left(1-\left(\mu_{\sigma(i)}^{-}\right)^{2}\right)^{\kappa_{i}}}, \sqrt{\left.1-\prod_{i=1}^{n}\left(1-\left(\mu_{\sigma(i)}^{+}\right)^{2}\right)^{\kappa_{i}}\right]},\left[\prod_{i=1}^{n}\left(v_{\sigma(i)}^{-}\right)^{\kappa_{i}}, \prod_{i=1}^{n}\left(v_{\sigma(i)}^{+}\right)^{\kappa_{i}}\right]\right\rangle\right. \\
& \left.\cdot \mid\left\langle\tilde{\mu}_{\sigma(i)}, \widetilde{v}_{\sigma(i)}\right\rangle \in \widetilde{\mathscr{P}}_{\sigma(i)}, i=1, \cdots, n\right\} .
\end{aligned}
$$

(2) An interval-valued Pythagorean hesitant fuzzy ordered weighted geometric (IVPHFOWG) operator can be seen a map IVPHFOWG: $\Omega^{n} \longrightarrow \Omega$, such that

$$
\begin{aligned}
& \operatorname{IVPHFOWG}\left(\widetilde{\mathscr{P}}_{1}, \cdots, \widetilde{\mathscr{P}}_{n}\right)=\widetilde{\mathscr{P}}_{\sigma(1)}^{\kappa_{1}} \otimes \cdots \otimes \widetilde{\mathscr{P}}_{\sigma(n)}^{\kappa_{n}} \\
& \begin{aligned}
= & \left\{\left\langle\left[\prod_{i=1}^{n}\left(\mu_{\sigma(i)}^{-}\right)^{\kappa_{i}}, \prod_{i=1}^{n}\left(\mu_{\sigma(i)}^{+}\right)^{\kappa_{i}}\right],\left[\sqrt{1-\prod_{i=1}^{n}\left(1-\left(v_{\sigma(i)}^{-}\right)^{2}\right)^{\kappa_{i}}}, \sqrt{1-\prod_{i=1}^{n}\left(1-\left(\nu_{\sigma(i)}^{+}\right)^{2}\right)^{\kappa_{i}}}\right]\right\rangle\right. \\
& \left.\cdot \mid\left\langle\widetilde{\mu}_{\sigma(i)}, \tilde{\nu}_{\sigma(i)}\right\rangle \in \widetilde{\mathscr{P}}_{\sigma(i)}, i=1, \cdots, n\right\} .
\end{aligned}
\end{aligned}
$$

(3) A generalized interval-valued Pythagorean hesitant fuzzy ordered weighted averaging (GIVPHFOWA) operator can be seen a map GIVPHFOWA: $\Omega^{n} \longrightarrow \Omega$, which satisfies

$$
\begin{aligned}
& \operatorname{GIVPHFOWA}_{\lambda}\left(\widetilde{\mathscr{P}}_{1}, \cdots, \widetilde{\mathscr{P}}_{n}\right)=\left(\kappa_{1} \dot{\tilde{\mathscr{P}}}_{\sigma(1)^{\lambda}} \oplus \cdots \oplus \kappa_{n} \dot{\widetilde{\mathscr{P}}}_{\sigma(n)}\right)^{1 / \lambda} \\
& =\left\{\left\langle\sqrt{\left(1-\prod_{i=1}^{n}\left(1-\left(\dot{\mu}_{\sigma(i)}^{-}\right)^{2 \lambda}\right)^{\kappa_{i}}\right)^{1 / \lambda}}, \sqrt{\left(1-\prod_{i=1}^{n}\left(1-\left(\dot{\mu}_{\sigma(i)}^{+}\right)^{2 \lambda}\right)^{\kappa_{i}}\right)^{1 / \lambda}}\right],\right. \\
& \left.\left[\sqrt{1-\left(1-\prod_{i=1}^{n}\left(1-\left(1-\left(\dot{v}_{\sigma(i)}^{-}\right)^{2}\right)^{\lambda}\right)^{\kappa_{i}}\right)^{1 / \lambda}}, \sqrt{1-\left(1-\prod_{i=1}^{n}\left(1-\left(1-\left(\dot{v}_{\sigma(i)}^{+}\right)^{2}\right)^{\lambda}\right)^{\kappa_{i}}\right)^{1 / \lambda}}\right]\right\rangle \text {, } \\
& \left.\cdot \mid\left\langle\dot{\tilde{\mu}}_{\sigma(i)} \dot{\tilde{v}}_{\sigma(i)}\right\rangle \in \dot{\tilde{\mathscr{P}}}_{\sigma(i)}, i=1, \cdots, n\right\} \text {, }
\end{aligned}
$$


where $\lambda>0$.

(4) A generalized interval-valued Pythagorean hesitant

operator can be seen a map fuzzy ordered weighted geometric (GIVPHFOWG)

GIVPHFOWG: $\Omega^{n} \longrightarrow \Omega$, which satisfies

$$
\begin{aligned}
& \operatorname{GIVPHFOWG}_{\lambda}\left(\widetilde{\mathscr{P}}_{1}, \cdots, \widetilde{\mathscr{P}}_{n}\right)=\frac{1}{\lambda}\left(\left(\lambda \ddot{\widetilde{P}}_{\sigma(1)}\right)^{\kappa_{1}} \otimes \cdots \otimes\left(\lambda \ddot{\widetilde{\mathscr{P}}}_{\sigma(n)}\right)^{\kappa_{n}}\right) \\
& =\left\{\left\langle\sqrt{1-\left(1-\prod_{i=1}^{n}\left(1-\left(1-\left(\ddot{\mu}_{\sigma(i)}^{-}\right)^{2}\right)^{\lambda}\right)^{\kappa_{i}}\right)^{1 / \lambda}}, \sqrt{1-\left(1-\prod_{i=1}^{n}\left(1-\left(1-\left(\ddot{\mu}_{\sigma(i)}^{+}\right)^{2}\right)^{\lambda}\right)^{\kappa_{i}}\right)^{1 / \lambda}}\right],\right. \\
& \left.\cdot\left[\sqrt{\left(1-\prod_{i=1}^{n}\left(1-\left(\ddot{\nu}_{\sigma(i)}^{-}\right)^{2 \lambda}\right)^{\kappa_{i}}\right)^{1 / \lambda}}, \sqrt{\left(1-\prod_{i=1}^{n}\left(1-\left(\ddot{v}_{\sigma(i)}^{+}\right)^{2 \lambda}\right)^{\kappa_{i}}\right)^{1 / \lambda}}\right]\right\rangle,
\end{aligned}
$$

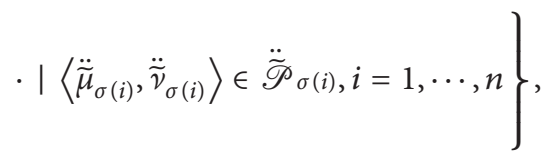

where $\lambda>0$. If $\lambda=1$, then the GIVPHFOWA and GIVPHFOWG operators reduce to the IVPHFOWA and IVPHFOWG operators, respectively.

By comparing Definition 17 with Definition 18, we can find that the IVPHFOWA, IVPHFOWG, GIVPHFOWA, and GIVPHFOWG operators weight the ordered positions of the IVPHFEs instead of weighting the IVPHFEs themselves. Also, their characteristics lie in reordering original IVPHFEs according to a decreasing order and in aggregating through the associated weights of positions where the weight $\kappa_{i}$ is associated with the $i^{\text {th }}$ position in the collection of the IVPHFEs during aggregation processes.

\section{Example 2. Suppose}

$$
\begin{aligned}
\widetilde{P}_{1}= & \{\langle[0.3,0.5],[0.2,0.4]\rangle\}, \\
\widetilde{\mathscr{P}}_{2}= & \{\langle[0.1,0.4],[0.3,0.6]\rangle,\langle[0.1,0.4],[0.5,0.7]\rangle\}, \\
\widetilde{\mathscr{P}}_{3}= & \{\langle[0.3,0.4],[0.2,0.6]\rangle,\langle[0.1,0.3],[0.2,0.6]\rangle, \\
& \langle[0.4,0.7],[0.2,0.6]\rangle\},
\end{aligned}
$$

are three IVPHFEs, and the aggregation-associated vector is $\kappa=(0.2,0.45,0.35)^{T}$.

Then, by Definition 15 , we have the score values of $\widetilde{\mathscr{P}}_{1}, \widetilde{\mathscr{P}}_{2}$, and $\widetilde{\mathscr{P}}_{3}$ as follows:

$$
\begin{aligned}
S\left(\widetilde{\mathscr{P}}_{1}\right) & =\left[\frac{1}{2}\left((0.3)^{2}-(0.4)^{2}\right), \frac{1}{2}\left((0.5)^{2}-(0.2)^{2}\right)\right]=[-0.035,0.045], \\
S\left(\widetilde{\mathscr{P}}_{2}\right) & =\left[\frac{1}{4}\left((0.1)^{2}-(0.6)^{2}+(0.1)^{2}-(0.7)^{2}\right), \frac{1}{4}\left((0.4)^{2}-(0.3)^{2}+(0.4)^{2}-(0.5)^{2}\right)\right] \\
& =[-0.2075,-0.005], \\
S\left(\widetilde{\mathscr{P}}_{3}\right) & =\left[\frac{1}{6}\left((0.3)^{2}-(0.6)^{2}+(0.1)^{2}-(0.6)^{2}+(0.4)^{2}-(0.6)^{2}\right), \frac{1}{6}\left((0.4)^{2}-(0.2)^{2}+(0.3)^{2}-(0.2)^{2}+(0.7)^{2}-(0.2)^{2}\right)\right] \\
& =[-0.1367,0.1033] .
\end{aligned}
$$

Based on Definition 16, we can obtain that $P\left(S\left(\widetilde{\mathscr{P}}_{1}\right)>S\left(\widetilde{\mathscr{P}}_{3}\right)\right)=0.5678$ and $P\left(S\left(\widetilde{\mathscr{P}}_{3}\right)>S\left(\widetilde{\mathscr{P}}_{2}\right)\right)=$ 0.7024 and then $\widetilde{\mathscr{P}}_{\sigma(1)}=\widetilde{\mathscr{P}}_{1}, \widetilde{\mathscr{P}}_{\sigma(2)}=\widetilde{\mathscr{P}}_{3}$, and $\widetilde{\mathscr{P}}_{\sigma(3)}=\widetilde{\mathscr{P}}_{2}$.
According to Definition 18, we have 
$\operatorname{IVPHFOWA}\left(\widetilde{\mathscr{P}}_{1}, \widetilde{\mathfrak{P}}_{2}, \widetilde{\mathscr{P}}_{3}\right)=\kappa_{1} \widetilde{\mathfrak{P}}_{\sigma(1)} \oplus \kappa_{1} \widetilde{\mathscr{P}}_{\sigma(2)} \oplus \kappa_{n} \widetilde{\mathscr{P}}_{\sigma(3)}$

$$
\begin{aligned}
= & \left\{\left\langle\sqrt{1-\prod_{i=1}^{3}\left(1-\left(\mu_{\sigma(i)}^{-}\right)^{2}\right)^{\kappa_{i}}}, \sqrt{1-\prod_{i=1}^{3}\left(1-\left(\mu_{\sigma(i)}^{+}\right)^{2}\right)^{\kappa_{i}}}\right],\left[\prod_{i=1}^{3}\left(v_{\sigma(i)}^{-}\right)^{\kappa_{i}}, \prod_{i=1}^{3}\left(v_{\sigma(i)}^{+}\right)^{\kappa_{i}}\right]\right\rangle \\
& \left.\cdot \mid\left\langle\tilde{\mu}_{\sigma(i)}, \widetilde{v}_{\sigma(i)}\right\rangle \in \widetilde{\mathscr{P}}_{\sigma(i)}, i=1,2,3\right\} .
\end{aligned}
$$

$=\{\langle[0.2505,0.4229],[0.2305,0.5533]\rangle,\langle[0.2505,0.4229],[0.2756,0.5839]\rangle,\langle[0.1629,0.3856]$, $[0.2305,0.5533]\rangle,\langle[0.1629,0.3856],[0.2756,0.5839]\rangle,\langle[0.3097,0.5865],[0.2305,0.5930]\rangle$, $\langle[0.3097,0.5865],[0.2756,0.6259]\rangle\} ;$

$\operatorname{IVPHFOWG}\left(\widetilde{\mathscr{P}}_{1}, \widetilde{\mathscr{P}}_{2}, \widetilde{\mathscr{P}}_{3}\right)=\widetilde{\mathscr{P}}_{\sigma(1)}^{\kappa_{1}} \otimes \widetilde{\mathscr{P}}_{\sigma(2)}^{\kappa_{2}} \otimes \widetilde{\mathscr{P}}_{\sigma(3)}^{\kappa_{3}}$

$$
\begin{aligned}
= & \left\{\left\langle\left[\prod_{i=1}^{3}\left(\mu_{\sigma(i)}^{-}\right)^{\kappa_{i}}, \prod_{i=1}^{3}\left(\mu_{\sigma(i)}^{+}\right)^{\kappa_{i}}\right],\left[\sqrt{1-\prod_{i=1}^{3}\left(1-\left(v_{\sigma(i)}^{-}\right)^{2}\right)^{\kappa_{i}}}, \sqrt{1-\prod_{i=1}^{3}\left(1-\left(v_{\sigma(i)}^{+}\right)^{2}\right)^{\kappa_{i}}}\right]\right\rangle\right. \\
& \left.\cdot \mid\left\langle\widetilde{\mu}_{\sigma(i)}, \tilde{v}_{\sigma(i)}\right\rangle \in \widetilde{P}_{\sigma(i)}, i=1,2,3\right\} . \\
= & \{\langle[0.2042,0.4183],[0.2404,0.5694]\rangle,\langle[0.2042,0.4183],[0.3456,0.6130]\rangle,\langle[0.1246,0.3675], \\
& {[0.2404,0.5694]\rangle,\langle[0.1246,0.3675],[0.3456,0.6131]\rangle,\langle[0.2325,0.5380],[0.2404,0.6244]\rangle, } \\
& \langle[0.2325,0.5380],[0.3456,0.6607]\rangle\} ;
\end{aligned}
$$

$\operatorname{GIVPHFOWA}_{3}\left(\widetilde{\mathscr{P}}_{1}, \widetilde{\mathscr{P}}_{2}, \widetilde{\mathscr{P}}_{3}\right)=\left(\kappa_{1} \widetilde{\mathscr{P}}_{\sigma(1)}^{3} \oplus \kappa_{2} \widetilde{\mathscr{P}}_{\sigma(2)}^{3} \oplus \kappa_{3} \widetilde{\mathscr{P}}_{\sigma(3)}^{3}\right)^{1 / 3}$

$$
\begin{aligned}
= & \left\langle\left\langle\sqrt{\left(1-\prod_{i=1}^{3}\left(1-\left(\mu_{\sigma(i)}^{-}\right)^{2 \times 3}\right)^{\kappa_{i}}\right)^{1 / 3}}, \sqrt{\left(1-\prod_{i=1}^{3}\left(1-\left(\mu_{\sigma(i)}^{+}\right)^{2 \times 3}\right)^{\kappa_{i}}\right)^{1 / 3}}\right],\right. \\
& \left.\cdot\left[\sqrt{1-\left(1-\prod_{i=1}^{3}\left(1-\left(1-\left(v_{\sigma(i)}^{-}\right)^{2}\right)^{3}\right)^{\kappa_{i}}\right)^{1 / 3}}, \sqrt{1-\left(1-\prod_{i=1}^{3}\left(1-\left(1-\left(\nu_{\sigma(i)}^{+}\right)^{2}\right)^{3}\right)^{\kappa_{i}}\right)^{1 / 3}}\right]\right\rangle, \\
& \left.\cdot \mid\left\langle\widetilde{\mu}_{\sigma(i)}, \widetilde{v}_{\sigma(i)}\right\rangle \in \widetilde{\mathscr{P}}_{\sigma(i)}, i=1,2,3\right\}, \\
= & \{\langle[0.2793,0.4310],[0.2299,0.5466]\rangle,\langle[0.2793,0.4310],[0.2697,0.5706]\rangle, \\
& \langle[0.2296,0.4122],[0.2300,0.5466]\rangle,\langle[0.2296,0.4122],[0.2697,0.5706]\rangle, \\
& \langle[0.3547,0.6241],[0.2299,0.5780]\rangle,\langle[0.3547,0.6241],[0.2697,0.6053]\rangle\} ;
\end{aligned}
$$

$\operatorname{GIVPHFOWG}\left(\widetilde{\mathscr{P}}_{1}, \widetilde{\mathscr{P}}_{2}, \widetilde{\mathscr{P}}_{3}\right)=\frac{1}{3}\left(\left(3 \widetilde{\mathscr{P}}_{\sigma(1)}\right)^{\kappa_{1}} \otimes\left(3 \widetilde{\mathscr{P}}_{\sigma(2)}\right)^{\kappa_{2}} \otimes\left(3 \widetilde{\mathscr{P}}_{\sigma(3)}\right)^{\kappa_{3}}\right)$

$$
\begin{aligned}
&=\left\{\left[\sqrt{1-\left(1-\prod_{i=1}^{3}\left(1-\left(1-\left(\mu_{\sigma(i)}^{-}\right)^{2}\right)^{3}\right)^{\kappa_{i}}\right)^{1 / 3}}, \sqrt{1-\left(1-\prod_{i=1}^{3}\left(1-\left(1-\left(\mu_{\sigma(i)}^{+}\right)^{2}\right)^{3}\right)^{\kappa_{i}}\right)^{1 / 3}}\right],\right. \\
&\left.\cdot\left[\sqrt{\left(1-\prod_{i=1}^{3}\left(1-\left(v_{\sigma(i)}^{-}\right)^{2 \times 3}\right)^{\kappa_{i}}\right)^{1 / 3}}, \sqrt{\left(1-\prod_{i=1}^{3}\left(1-\left(\nu_{\sigma(i)}^{+}\right)^{2 \times 3}\right)^{\kappa_{i}}\right)^{1 / 3}}\right]\right\rangle, \\
&\left.\cdot \mid\left\langle\widetilde{\mu}_{\sigma(i)}, \widetilde{v}_{\sigma(i)}\right\rangle \in \widetilde{\mathscr{P}}_{\sigma(i)}, i=1,2,3\right\}, \\
&=\{\langle[0.2020,0.4174],[0.2583,0.5806]\rangle,\langle[0.2020,0.4174],[0.4206,0.6324]\rangle, \\
&\langle[0.1239,0.3648],[0.2583,0.5806]\rangle,\langle[0.1239,0.3648],[0.4206,0.6324]\rangle, \\
&\langle[0.2275,0.5213],[0.2583,0.6438]\rangle,\langle[0.2275,0.5213],[0.4206,0.6767]\rangle\} .
\end{aligned}
$$


Based on Definition 14, we can obtain that

$$
\begin{aligned}
& S\left(\operatorname{IVPHFOWA}\left(\widetilde{\mathscr{P}}_{1}, \widetilde{\mathscr{P}}_{2}, \widetilde{\mathscr{P}}_{3}\right)\right)=[-0.1389,0.0796], \\
& S\left(\operatorname{IVPHFOWG}\left(\widetilde{\mathscr{P}}_{1}, \widetilde{\mathscr{P}}_{2}, \widetilde{\mathscr{P}}_{3}\right)\right)=[-0.1670,0.0556], \\
& S\left(\operatorname{GIVPHFOWA}{ }_{3}\left(\widetilde{\mathscr{P}}_{1}, \widetilde{\mathscr{P}}_{2}, \widetilde{\mathscr{P}}_{3}\right)\right)=[-0.1197,0.1001] \text {, } \\
& S\left(\operatorname{GIVPHFOWG}{ }_{3}\left(\widetilde{\mathscr{P}}_{1}, \widetilde{\mathscr{P}}_{2}, \widetilde{\mathscr{P}}_{3}\right)\right)=[-0.1776,0.0356] \text {. }
\end{aligned}
$$

Then,

$$
\begin{aligned}
& P\left(S\left(\operatorname{IVPHFOWG}\left(\widetilde{\mathscr{P}}_{1}, \widetilde{\mathscr{P}}_{2}, \widetilde{\mathscr{P}}_{3}\right)\right) \leq S\left(\operatorname{IVPHFOWA}\left(\widetilde{\mathscr{P}}_{1}, \widetilde{\mathscr{P}}_{2}, \widetilde{\mathscr{P}}_{3}\right)\right)\right)=0.5591, \\
& P\left(S\left(\operatorname{IVPHFOWG}\left(\widetilde{\mathscr{P}}_{1}, \widetilde{\mathscr{P}}_{2}, \widetilde{\mathscr{P}}_{3}\right)\right) \leq S\left(\operatorname{GIVPHFOWA}{ }_{3}\left(\widetilde{\mathscr{P}}_{1}, \widetilde{\mathscr{P}}_{2}, \widetilde{\mathscr{P}}_{3}\right)\right)\right)=0.6038 \text {, } \\
& P\left(S\left(\operatorname{GIVPHFOWG}_{3}\left(\widetilde{\mathscr{P}}_{1}, \widetilde{\mathscr{P}}_{2}, \widetilde{\mathscr{P}}_{3}\right)\right) \leq S\left(\operatorname{IVPHFOWA}\left(\widetilde{\mathscr{P}}_{1}, \widetilde{\mathscr{P}}_{2}, \widetilde{\mathscr{P}}_{3}\right)\right)\right)=0.5958 .
\end{aligned}
$$

So,

$\operatorname{IVPHFOWG}\left(\widetilde{\mathscr{P}}_{1}, \widetilde{\mathscr{P}}_{2}, \widetilde{\mathscr{P}}_{3}\right) \preccurlyeq \operatorname{IVPHFOWA}\left(\widetilde{\mathscr{P}}_{1}, \widetilde{\mathscr{P}}_{2}, \widetilde{\mathscr{P}}_{3}\right)$, $\operatorname{IVPHFOWG}\left(\widetilde{\mathscr{P}}_{1}, \tilde{\mathscr{P}}_{2}, \widetilde{\mathscr{P}}_{3}\right) \leqslant \operatorname{GIVPHFOWA}\left(\tilde{\mathscr{P}}_{1}, \tilde{\mathscr{P}}_{2}, \widetilde{\mathscr{P}}_{3}\right)$, $\operatorname{GIVPHFOWG}\left(\widetilde{\mathscr{P}}_{1}, \widetilde{\mathscr{P}}_{2}, \widetilde{\mathscr{P}}_{3}\right) \leqslant \operatorname{IVPHFOWA}\left(\widetilde{\mathscr{P}}_{1}, \widetilde{\mathscr{P}}_{2}, \widetilde{\mathscr{P}}_{3}\right)$.
Similar to Proposition 5-7, we easily obtain the following properties of ordered aggregation operators of IVPHFEs.

Proposition 8. Let $\widetilde{\mathscr{P}}_{i}(i=1,2, \cdots, n)$ be a collection of IVPHFEs, $\lambda>0$, and $\lambda_{2}>\lambda_{1}>0$, then

$$
\begin{aligned}
& \operatorname{IVPHFOWG}\left(\widetilde{\mathscr{P}}_{1}, \widetilde{\mathscr{P}}_{2}, \cdots, \widetilde{\mathscr{P}}_{n}\right) \preccurlyeq \operatorname{IVPHFOWA}\left(\widetilde{\mathscr{P}}_{1}, \widetilde{\mathscr{P}}_{2}, \cdots, \widetilde{\mathscr{P}}_{n}\right) \text {, } \\
& \operatorname{IVPHFOWG}\left(\widetilde{\mathscr{P}}_{1}, \widetilde{\mathscr{P}}_{2}, \cdots, \widetilde{\mathscr{P}}_{n}\right) \leqslant \operatorname{GIVPHFOWA}\left(\widetilde{\mathscr{P}}_{1}, \widetilde{\mathscr{P}}_{2}, \cdots, \widetilde{\mathscr{P}}_{n}\right) \text {, } \\
& \operatorname{GIVPHFOWG}_{\lambda}\left(\widetilde{\mathscr{P}}_{1}, \widetilde{\mathscr{P}}_{2}, \cdots, \widetilde{\mathscr{P}}_{n}\right) \preccurlyeq \operatorname{IVPHFOWA}\left(\widetilde{\mathscr{P}}_{1}, \widetilde{\mathscr{P}}_{2}, \cdots, \widetilde{\mathscr{P}}_{n}\right) \text {, }
\end{aligned}
$$

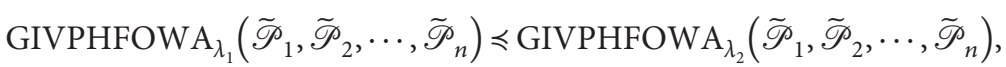

$$
\begin{aligned}
& \operatorname{GIVPHFOWG}_{\lambda_{1}}\left(\widetilde{\mathscr{P}}_{1}, \widetilde{\mathscr{P}}_{2}, \cdots, \widetilde{\mathscr{P}}_{n}\right) \geqslant \operatorname{GIVPHFOWG}_{\lambda_{2}}\left(\widetilde{\mathscr{P}}_{1}, \widetilde{\mathscr{P}}_{2}, \cdots, \widetilde{\mathscr{P}}_{n}\right) \text {. }
\end{aligned}
$$

From Definition 17 and Definition 18, we know that the IVPHFWA, IVPHFWG, GIVPHFWA, and GIVPHFWG operators weight the interval-valued hesitant fuzzy argument itself and there is no consideration of the impact of the order of parameters, while the IVPHFOWA, IVPHFOWG, GIVPHFOWA, and GIVPHFOWG operators only weight the ordered position of each given IVPHFE and there is no consideration of the impact of the argument itself. Hence, we propose a new hybrid aggregation operator which can weight both all the given arguments and their ordered positions.
4.3. The IVPHFHA, IVPHFHG, GIVPHFHA, and GIVPHFHG Operators

Definition 19. For a collection of some IVPHFEs $\widetilde{\mathscr{P}}_{i}(i=1,2, \cdots, n), \quad \omega=\left(\omega_{1}, \omega_{2}, \cdots, \omega_{n}\right)^{T}$ is the weight vector of them with $\omega_{i} \in[0,1]$ and $\sum_{i=1}^{n} \omega_{i}=1$. Suppose $\kappa=$ $\left(\kappa_{1}, \kappa_{2}, \cdots, \kappa_{n}\right)^{T}$ is the associated vector such that $\kappa_{i} \in[0,1]$, $\sum_{i=1}^{n} \kappa_{i}=1$, and $\lambda>0$. Then,

(1) An interval-valued Pythagorean hesitant fuzzy hybrid averaging (IVPHFHA) operator can be seen a map IVPHFHA: $\Omega^{n} \longrightarrow \Omega$, such that

$$
\begin{aligned}
& \operatorname{IVPHFHA}\left(\widetilde{\mathscr{P}}_{1}, \cdots, \widetilde{\mathscr{P}}_{n}\right)=\kappa_{1} \dot{\mathscr{\mathscr { P }}}_{\sigma(1)} \oplus \cdots \oplus \kappa_{n} \dot{\mathscr{\mathscr { P }}}_{\sigma(n)}=\left\{\left\langle\left[\sqrt{1-\prod_{i=1}^{n}\left(1-\left(\dot{\mu}_{\sigma(i)}^{-}\right)^{2}\right)^{\kappa_{i}}}, \sqrt{1-\prod_{i=1}^{n}\left(1-\left(\dot{\mu}_{\sigma(i)}^{+}\right)^{2}\right)^{\kappa_{i}}},\right.\right.\right. \\
& \left.\left.\left[\prod_{i=1}^{n}\left(\dot{v}_{\sigma(i)}^{-}\right)^{\kappa_{i}}, \prod_{i=1}^{n}\left(\dot{v}_{\sigma(i)}^{+}\right)^{\kappa_{i}}\right]\right\rangle \mid\left\langle\dot{\tilde{\mu}}_{\sigma(i)}, \dot{\tilde{v}}_{\sigma(i)}\right\rangle \in \dot{\tilde{\mathscr{P}}}_{\sigma(i)}, i=1, \cdots, n\right\} \text {, }
\end{aligned}
$$


16

Complexity

where $\dot{\mathscr{\mathscr { T }}}_{\sigma(i)}$ is the largest $i^{\text {th }}$ of $\dot{\mathscr{\mathscr { T }}}_{k}=n \omega_{k} \widetilde{\mathscr{P}}_{k}(k=1,2, \cdots, n)$.

(2) An interval-valued Pythagorean hesitant fuzzy bybid geometric (IVPHFHG) operator can be seen a map IVPHFHG: $\Omega^{n} \longrightarrow \Omega$, such that

$$
\begin{aligned}
& \operatorname{IVPHFHG}\left(\widetilde{\mathscr{P}}_{1}, \cdots, \widetilde{\mathscr{P}}_{n}\right)=\left(\ddot{\tilde{\mathscr{P}}}_{\sigma(1)}\right)^{\kappa_{1}} \otimes \cdots \otimes\left({ }_{\sigma(n)}\right)^{\kappa_{n}} \\
& =\left\{\left\langle\left[\prod_{i=1}^{n}\left(\ddot{\mu}_{\sigma(i)}^{-}\right)^{\kappa_{i}}, \prod_{i=1}^{n}\left(\ddot{\mu}_{\sigma(i)}^{+}\right)^{\kappa_{i}}\right],\left[\sqrt{1-\prod_{i=1}^{n}\left(1-\left(\ddot{v}_{\sigma(i)}^{-}\right)^{2}\right)^{\kappa_{i}}}, \sqrt{1-\prod_{i=1}^{n}\left(1-\left(\ddot{v}_{\sigma(i)}^{+}\right)^{2}\right)^{\kappa_{i}}}\right]\right\rangle\right. \\
& \left.\mid\left\langle\ddot{\widetilde{\mu}}_{\sigma(i)}, \ddot{\widetilde{v}}_{\sigma(i)}\right\rangle \in \ddot{\widetilde{\mathscr{P}}}_{\sigma(i)}, i=1, \cdots, n\right\} .
\end{aligned}
$$

where $\ddot{\mathscr{T}}_{\sigma(i)}$ is the largest $i^{\text {th }}$ of $\quad \ddot{\mathscr{P}}_{k}=\left(\widetilde{\mathscr{P}}_{k}\right)^{n \omega_{k}}$ $(k=1,2, \cdots, n)$. be seen a map GIVPHFHA: $\Omega^{n} \longrightarrow \Omega$, which satisfies

(3) A generalized interval-valued Pythagorean hesitant fuzzy hybrid averaging (GIVPHFHA) operator can

$$
\begin{aligned}
& \operatorname{GIVPHFHA}_{\lambda}\left(\widetilde{\mathscr{P}}_{1}, \cdots, \widetilde{\mathscr{P}}_{n}\right)=\left(\kappa_{1}\left(\dot{\tilde{\mathscr{P}}}_{\sigma(1)}\right)^{\lambda} \oplus \cdots \oplus \kappa_{n}\left(\dot{\widetilde{\mathscr{P}}}_{\sigma(n)}\right)^{\lambda}\right)^{1 / \lambda} \\
& =\left\{\left\langle\sqrt{\left(1-\prod_{i=1}^{n}\left(1-\left(\dot{\mu}_{\sigma(i)}^{-}\right)^{2 \lambda}\right)^{\kappa_{i}}\right)^{1 / \lambda}}, \sqrt{\left(1-\prod_{i=1}^{n}\left(1-\left(\dot{\mu}_{\sigma(i)}^{+}\right)^{2 \lambda}\right)^{\kappa_{i}}\right)^{1 / \lambda}}\right],\right. \\
& \left.\left[\sqrt{1-\left(1-\prod_{i=1}^{n}\left(1-\left(1-\left(\dot{v}_{\sigma(i)}^{-}\right)^{2}\right)^{\lambda}\right)^{\kappa_{i}}\right)^{1 / \lambda}}, \sqrt{1-\left(1-\prod_{i=1}^{n}\left(1-\left(1-\left(\dot{v}_{\sigma(i)}^{+}\right)^{2}\right)^{\lambda}\right)^{\kappa_{i}}\right)^{1 / \lambda}}\right]\right\rangle \text {, } \\
& \left.\mid\left\langle\dot{\tilde{\mu}}_{\sigma(i)}, \dot{\tilde{v}}_{\sigma(i)}\right\rangle \in \dot{\mathscr{\mathscr { P }}}_{\sigma(i)}, i=1, \cdots, n\right\} \text {, }
\end{aligned}
$$

where $\dot{\mathscr{\mathscr { T }}}_{\sigma(i)}$ is the largest $i^{\text {th }}$ of $\dot{\mathscr{\mathscr { T }}}_{k}=n \omega_{k} \widetilde{\mathscr{P}}_{k}(k=1,2, \cdots, n)$.

(4) A generalized interval-valued Pythagorean hesitant fuzzy hybrid geometric (GIVPHFHG) operator can be seen a map GIVPHFHG: $\Omega^{n} \longrightarrow \Omega$, which satisfies

$$
\begin{aligned}
& \operatorname{GIVPHFHG}_{\lambda}\left(\widetilde{\mathscr{P}}_{1}, \cdots, \widetilde{\mathscr{P}}_{n}\right)=\frac{1}{\lambda}\left(\left(\lambda \ddot{\mathscr{\mathscr { P }}}_{\sigma(1)}\right)^{\kappa_{1}} \otimes \cdots \otimes\left(\lambda \ddot{\mathscr{\mathscr { P }}}_{\sigma(n)}\right)^{\kappa_{n}}\right) \\
& =\left\{\left\langle\sqrt{1-\left(1-\prod_{i=1}^{n}\left(1-\left(1-\left(\ddot{\mu}_{\sigma(i)}^{-}\right)^{2}\right)^{\lambda}\right)^{\kappa_{i}}\right)^{1 / \lambda}}, \sqrt{1-\left(1-\prod_{i=1}^{n}\left(1-\left(1-\left(\ddot{\mu}_{\sigma(i)}^{+}\right)^{2}\right)^{\lambda}\right)^{\kappa_{i}}\right)^{1 / \lambda}}\right],\right. \\
& \left.\left[\sqrt{\left(1-\prod_{i=1}^{n}\left(1-\left(\ddot{\nu}_{\sigma(i)}^{-}\right)^{2 \lambda}\right)^{\kappa_{i}}\right)^{1 / \lambda}}, \sqrt{\left(1-\prod_{i=1}^{n}\left(1-\left(\ddot{\nu}_{\sigma(i)}^{+}\right)^{2 \lambda}\right)^{\kappa_{i}}\right)^{1 / \lambda}}\right]\right\rangle \text {, } \\
& \left.\mid\left\langle\dot{\tilde{\mu}}_{\sigma(i)}, \dot{\tilde{v}}_{\sigma(i)}\right\rangle \in \ddot{\widetilde{P}}_{\sigma(i)}, i=1, \cdots, n\right\} \text {, }
\end{aligned}
$$

where $\ddot{\mathscr{P}}_{\sigma(i)}$ is the largest $i^{\text {th }}$ of $\dot{\mathscr{\mathscr { T }}}_{k}=\left(\widetilde{\mathscr{P}}_{k}\right)^{n \omega_{k}}(k=1,2, \cdots, n)$. 
If $\lambda=1$, then the GIVPHFHA and GIVPHFHG operators reduce to the IVPHFHA and IVPHFHG operators, respectively.

$$
\begin{aligned}
& \widetilde{\mathscr{P}}_{2}=\{\langle[0.1,0.4],[0.3,0.6]\rangle,\langle[0.1,0.4],[0.5,0.7]\rangle\}, \\
& \widetilde{P}_{3}=\{\langle[0.3,0.4],[0.2,0.6]\rangle,\langle[0.1,0.3],[0.2,0.6]\rangle,\langle[0.4,0.7],[0.2,0.6]\rangle\},
\end{aligned}
$$

are three IVPHFEs, the weight vector of the attributes is $\omega=(0.15,0.30,0.55)^{T}$, and the aggregation-associated vector is $\kappa=(0.2,0.45,0.35)^{T}$. Then, we can obtain

$$
\begin{aligned}
\dot{\mathscr{P}}_{1}= & (3 \times 0.15) \otimes \widetilde{\mathscr{P}}_{1}=\{\langle[0.2038,0.3485],[0.4847,0.6621]\rangle\}, \\
\dot{\mathscr{P}}_{2}= & (3 \times 0.3) \otimes \widetilde{\mathscr{P}}_{2}=\{\langle[0.0949,0.3811],[0.3384,0.6314]\rangle,\langle[0.0949,0.3811],[0.5359,0.7254]\rangle\}, \\
\dot{\widetilde{\mathscr{P}}}_{3}= & (3 \times 0.3) \otimes \widetilde{\mathscr{P}}_{3}=\{\langle[0.3796,0.5000],[0.0703,0.4305]\rangle,\langle[0.1282,0.3796],[0.0703,0.4305]\rangle, \\
& \langle[0.5000,0.8190],[0.0703,0.4305]\rangle\} . \\
\ddot{\widetilde{P}}_{1}= & \left(\widetilde{\mathscr{P}}_{1}\right)^{3 \times 0.15}=\{\langle[0.5817,0.7320],[0.1349,0.2747]\rangle\}, \\
\ddot{\mathscr{P}}_{2}= & \left(\widetilde{\mathscr{P}}_{2}\right)^{3 \times 0.3}=\{\langle[0.1259,0.4384],[0.2853,0.5751]\rangle,\langle[0.1259,0.4384],[0.4776,0.6741]\rangle\}, \\
\ddot{\mathscr{P}}_{3}= & \left(\widetilde{\mathscr{P}}_{3}\right)^{3 \times 0.3}=\{\langle[0.1372,0.2205],[0.2552,0.7219]\rangle,\langle[0.0224,0.1372],[0.2552,0.7219]\rangle, \\
& \langle[0.2205,0.5552],[0.2552,0.7219]\rangle\} . \\
S\left(\dot{\mathscr{\mathscr { P }}}_{1}\right)= & {[-0.1984,-0.0567], S\left(\dot{\mathscr{\mathscr { P }}}_{2}\right)=[-0.2267,-0.0278], } \\
S\left(\dot{\mathscr{\mathscr { P }}}_{3}\right)= & {[-0.0242,0.1750], S\left(\ddot{\mathscr{P}}_{1}\right)=[0.1315,0.2588], } \\
S\left(\ddot{\mathscr{P}}_{2}\right)= & {[-0.1884,0.0187], S\left(\ddot{\mathscr{P}}_{3}\right)=[-0.2493,0.0300] . }
\end{aligned}
$$

By Definition 16, we have $P\left(S\left(\dot{\widetilde{\mathscr{T}}}_{1}\right) \leq S\left(\dot{\widetilde{\mathscr{T}}}_{2}\right)\right)=1$, $P\left(S\left(\dot{\mathscr{\mathscr { P }}}_{2}\right) \leq S\left(\dot{\mathscr{\mathscr { P }}}_{3}\right)\right)=0.5009$, and $P\left(S\left(\ddot{\mathscr{\mathscr { P }}}_{3}\right) \leq S\left(\ddot{\mathscr{\mathscr { P }}}_{2}\right)\right)=$ 0.5509 , and $P\left(S\left(\dot{\mathscr{\mathscr { P }}}_{2}\right) \leq S\left(\ddot{\mathscr{\mathscr { P }}}_{1}\right)\right)=1$. So, we can get

$$
\begin{aligned}
& \dot{\widetilde{\mathscr{P}}}_{\sigma(1)}=\dot{\widetilde{\mathscr{P}}}_{3}, \\
& \dot{\tilde{\mathscr{P}}}_{\sigma(2)}=\dot{\widetilde{\mathscr{P}}}_{2}, \\
& \dot{\mathscr{\mathscr { P }}}_{\sigma(3)}=\dot{\mathscr{\mathscr { P }}}_{1}, \\
& \ddot{\widetilde{\mathscr{P}}}_{\sigma(1)}=\ddot{\widetilde{\mathscr{P}}}_{1}, \\
& \ddot{\tilde{\mathscr{P}}}_{\sigma(2)}=\ddot{\widetilde{\mathscr{P}}}_{2}, \\
& \ddot{\widetilde{\mathscr{P}}}_{\sigma(3)}=\ddot{\widetilde{\mathscr{P}}}_{3} .
\end{aligned}
$$


According to Definition 19, we can obtain

$$
\begin{aligned}
& \operatorname{IVPHFHA}\left(\widetilde{\mathscr{P}}_{1}, \widetilde{\mathscr{P}}_{2}, \widetilde{\mathscr{P}}_{3}\right)=\kappa_{1} \dot{\widetilde{\mathscr{P}}}_{\sigma(1)} \oplus \kappa_{1} \dot{\widetilde{\mathscr{P}}}_{\sigma(2)} \oplus \kappa_{n} \dot{\widetilde{\mathscr{P}}}_{\sigma(3)} \\
& =\left\{\left\langle\left[\sqrt{1-\prod_{i=1}^{3}\left(1-\left(\dot{\mu}_{\sigma(i)}^{-}\right)^{2}\right)^{\kappa_{i}}}, \sqrt{1-\prod_{i=1}^{3}\left(1-\left(\dot{\mu}_{\sigma(i)}^{+}\right)^{2}\right)^{\kappa_{i}}}\right],\left[\prod_{i=1}^{3}\left(\dot{v}_{\sigma(i)}^{-}\right)^{\kappa_{i}}, \prod_{i=1}^{3}\left(\dot{v}_{\sigma(i)}^{+}\right)^{\kappa_{i}}\right]\right\rangle\right. \\
& \left.\mid\left\langle\dot{\tilde{\mu}}_{\sigma(i)} \dot{\widetilde{v}}_{\sigma(i)}\right\rangle \in \dot{\mathscr{P}}_{\sigma(i)}, i=1,2,3\right\} \\
& =\{\langle[0.2209,0.3991],[0.2802,0.5947]\rangle,\langle[0.2209,0.3991],[0.3446,0.6330]\rangle, \\
& \langle[0.1483,0.3698],[0.2802,0.5947]\rangle,\langle[0.1483,0.3698],[0.3446,0.6330]\rangle \text {, } \\
& \langle[0.2713,0.5356],[0.2802,0.5947]\rangle,\langle[0.2713,0.5356],[0.3446,0.6330]\rangle\} ; \\
& \operatorname{IVPHFHG}\left(\widetilde{\mathscr{T}}_{1}, \widetilde{\mathscr{P}}_{2}, \widetilde{\mathscr{P}}_{3}\right)=\left(\ddot{\mathscr{P}}_{\sigma(1)}\right)^{\kappa_{1}} \otimes\left(\ddot{\mathscr{P}}_{\sigma(2)}\right)^{\kappa_{2}} \otimes\left(\ddot{\mathscr{P}}_{\sigma(3)}\right)^{\kappa_{3}} \\
& =\left\{\left\langle\left[\prod_{i=1}^{3}\left(\ddot{\mu}_{\sigma(i)}^{-}\right)^{\kappa_{i}}, \prod_{i=1}^{3}\left(\ddot{\mu}_{\sigma(i)}^{+}\right)^{\kappa_{i}}\right],\left[\sqrt{1-\prod_{i=1}^{3}\left(1-\left(\ddot{\nu}_{\sigma(i)}^{-}\right)^{2}\right)^{\kappa_{i}}}, \sqrt{1-\prod_{i=1}^{3}\left(1-\left(\ddot{\nu}_{\sigma(i)}^{+}\right)^{2}\right)^{\kappa_{i}}}\right]\right\rangle\right. \\
& \left.\mid\left\langle\ddot{\tilde{\mu}}_{\sigma(i)} \ddot{\widetilde{v}}_{\sigma(i)}\right\rangle \in \ddot{\widetilde{P}}_{\sigma(i)}, i=1,2,3\right\} \\
& =\{\langle[0.1762,0.3819],[0.2517,0.6042]\rangle,\langle[0.0934,0.3235],[0.2517,0.6042]\rangle \text {, } \\
& \langle[0.2080,0.5276],[0.2517,0.6042]\rangle,\langle[0.1762,0.3819],[0.3659,0.6487]\rangle \text {, } \\
& \langle[0.0934,0.3234],[0.3659,0.6487]\rangle,\langle[0.2080,0.5276],[0.3659,0.6487]\rangle\} \text {; }
\end{aligned}
$$

$$
\begin{aligned}
& \operatorname{GIVPHFHA}_{3}\left(\widetilde{\mathscr{P}}_{1}, \widetilde{\mathscr{P}}_{2}, \widetilde{\mathscr{P}}_{3}\right)=\left(\kappa_{1}\left(\dot{\tilde{\mathscr{P}}}_{\sigma(1)}\right)^{3} \oplus \kappa_{2}\left(\dot{\mathscr{\mathscr { P }}}_{\sigma(2)}\right)^{3} \oplus \kappa_{3}\left(\dot{\mathscr{\mathscr { P }}}_{\sigma(3)}\right)^{3}\right)^{1 / 3} \\
& =\left\{\left\langle\sqrt{\left(1-\prod_{i=1}^{3}\left(1-\left(\dot{\mu}_{\sigma(i)}^{-}\right)^{2 \times 3}\right)^{\kappa_{i}}\right)^{1 / 3}}, \sqrt{\left(1-\prod_{i=1}^{3}\left(1-\left(\dot{\mu}_{\sigma(i)}^{+}\right)^{2 \times 3}\right)^{\kappa_{i}}\right)^{1 / 3}}\right],\right. \\
& \left.\left[\sqrt{1-\left(1-\prod_{i=1}^{3}\left(1-\left(1-\left(\dot{v}_{\sigma(i)}^{-}\right)^{2}\right)^{3}\right)^{\kappa_{i}}\right)^{1 / 3}}, \sqrt{1-\left(1-\prod_{i=1}^{3}\left(1-\left(1-\left(\dot{v}_{\sigma(i)}^{+}\right)^{2}\right)^{3}\right)^{\kappa_{i}}\right)^{1 / 3}}\right]\right\rangle, \\
& \left.\mid\left\langle\tilde{\mu}_{\sigma(i)}, \dot{v}_{\sigma(i)}\right\rangle \in \dot{\mathscr{P}}_{\sigma(i)}, i=1,2,3\right\} \\
& =\{\langle[0.2924,0.4155],[0.2713,0.5854]\rangle,\langle[0.2924,0.4155],[0.3255,0.6153]\rangle, \\
& \langle[0.1725,0.3709],[0.2713,0.5854]\rangle,\langle[0.1725,0.3709],[0.3256,0.6153]\rangle \text {, } \\
& \langle[0.3833,0.6438],[0.2713,0.5854]\rangle,\langle[0.3833,0.6438],[0.3256,0.6153]\rangle\} ; \\
& \operatorname{GIVPHFHG}_{3}\left(\widetilde{\mathscr{P}}_{1}, \widetilde{\mathscr{P}}_{2}, \widetilde{\mathscr{P}}_{3}\right)=\frac{1}{3}\left(\left(3 \ddot{\widetilde{P}}_{\sigma(1)}\right)^{\kappa_{1}} \otimes\left(3 \ddot{\widetilde{P}}_{\sigma(2)}\right)^{\kappa_{2}} \otimes\left(3 \ddot{\widetilde{P}}_{\sigma(3)}\right)^{\kappa_{3}}\right)
\end{aligned}
$$$$
=\left\{\left\langle\sqrt{1-\left(1-\prod_{i=1}^{3}\left(1-\left(1-\left(\ddot{\mu}_{\sigma(i)}^{-}\right)^{2}\right)^{3}\right)^{\kappa_{i}}\right)^{1 / 3}}, \sqrt{1-\left(1-\prod_{i=1}^{3}\left(1-\left(1-\left(\ddot{\mu}_{\sigma(i)}^{+}\right)^{2}\right)^{3}\right)^{\kappa_{i}}\right)^{1 / 3}}\right],\right.
$$$$
\left.\left[\sqrt{\left(1-\prod_{i=1}^{3}\left(1-\left(\ddot{\gamma}_{\sigma(i)}^{-}\right)^{2 \times 3}\right)^{\kappa_{i}}\right)^{1 / 3}}, \sqrt{\left(1-\prod_{i=1}^{3}\left(1-\left(\ddot{\gamma}_{\sigma(i)}^{+}\right)^{2 \times 3}\right)^{\kappa_{i}}\right)^{1 / 3}}\right]\right\rangle,
$$$$
\left.\mid\left\langle\ddot{\widetilde{\mu}}_{\sigma(i)}, \ddot{\widetilde{v}}_{\sigma(i)}\right\rangle \in \ddot{\mathscr{P}}_{\sigma(i)}, i=1,2,3\right\}
$$$$
=\{\langle[0.1714,0.3659],[0.2642,0.6384]\rangle,\langle[0.0902,0.3050],[0.2642,0.6384]\rangle \text {, }
$$$$
\langle[0.2024,0.5174],[0.2642,0.6384]\rangle,\langle[0.1714,0.3659],[0.4196,0.6734]\rangle \text {, }
$$$$
\langle[0.0902,0.3050],[0.4196,0.6734]\rangle,\langle[0.2024,0.5174],[0.4196,0.6734]\rangle\} .
$$ 


\section{The Application of the Aggregation Operators of IVPHFEs for MAGDM}

In multiattribute group decision-making (MAGDM) problems, because the different decision makers usually come from different research directions and backgrounds, they usually have diverging opinions. Thus, how to construct an optimal model that can be obtained with the maximum degree of consensus or agreement of these experts for the given alternatives is an important and interesting research content in MAGDM. In this section, we propose a new MAGDM method based on the interval-valued Pythagoras hesitant fuzzy integration operators. Furthermore, the ranking of integrated interval-valued Pythagorean hesitant fuzzy numbers is accomplished by adopting the score function. The specific details of the MAGDM method is shown in following.

Let $Y=\left\{Y_{i} \mid i=1,2, \cdots, m\right\}$ be a finite set of alternatives, $C=\left\{C_{j} \mid j=1,2, \cdots, n\right\} \quad$ be a set of attributes, and $D=\left\{D_{k} \mid k=1,2, \cdots, l\right\}$ be a set of $l$ decision makers. Then, let $M^{(k)}=\left(\widetilde{\mathscr{P}}_{i j}^{(k)}\right)_{m \times n}$ is an interval-valued Pythagorean hesitant fuzzy decision matrix (IVPHFDM) of the $k^{\text {th }}$ decision maker, where $\widetilde{\mathscr{P}}_{i j}^{(k)}=\left\{\left\langle\widetilde{\mu}_{i j}^{(k)}, \widetilde{v}_{i j}^{(k)}\right\rangle\right\},(i=1,2, \cdots, m$, $j=1,2, \cdots, n)$ is an IVPHFE given by the decision maker $D_{K}$, in which $\widetilde{\mu}_{i j}^{(k)}$ indicates the possible membership intervals that the alternative $Y_{i}$ satisfies the attribute $C_{j}$ and $\widetilde{v}_{i j}^{(k)}$ indicates the possible nonmembership intervals that the alternative $Y_{i}$ nonmembership intervals not satisfy the attribute $C_{j}$.
In the actual MAGDM problem, we will encounter some attributes are benefit (i.e., the bigger the attribute values, the better) and the other attributes are cost (i.e., the smaller the attribute values, the better). In such cases, transform the cost attribute values into the benefit attribute values and normalize the IVPHFDM $M^{(k)}=\left(\widetilde{\mathscr{P}}_{i j}^{(k)}\right)_{m \times n}$ into the corresponding IVPHFDM $N^{(k)}=\left(\widetilde{P}_{i j}^{(k)}\right)_{m \times n}$ by the method in [53] where

$$
\widetilde{P}_{i j}^{(k)}= \begin{cases}\widetilde{\mathscr{P}}_{i j}^{(k)}, & \text { for bene fit attribute } C_{j}, \\ \left(\widetilde{\mathscr{P}}_{i j}^{(k)}\right)^{C}, & \text { for cost attribute } C_{j},\end{cases}
$$

where $\left(\widetilde{\mathscr{P}}_{i j}^{(k)}\right)^{C}$ is the complement of $\widetilde{\mathscr{P}}_{i j}^{(k)}$.

Based on the above discussion and analysis, we develop an approach for multiattribute decision-making in intervalvalued Pythagorean hesitant fuzzy environments. The algorithm involves the following steps:

Step 1: construct the IVPHFDM $M^{(k)}=\left(\widetilde{\mathscr{P}}_{i j}^{(k)}\right)_{m \times n}$ and transform $M^{(k)}$ into the corresponding normalized matrix $N^{(k)}=\left(\tilde{\mathscr{P}}_{i j}^{(k)}\right)_{m \times n}$.

Step 2: utilize the GIVPHFWA operator (or the GIVPHFWG operator) to aggregate all the IVPHFDMs $N^{(k)}=\left(\widetilde{P}_{i j}^{(k)}\right)_{m \times n}\left(\widetilde{P}_{i j}=1,2, \cdots, l\right)$ into the collective IVPHFDM $N=\left(\widetilde{P}_{i j}\right)_{m \times n}$. where $\xi=\left(\xi_{1}, \xi_{2}, \cdots, \xi_{l}\right)^{T}$ is the weight vector of the decision makers $D_{k}(k=1,2, \cdots, l)$, and the specific integration operation is as follows:

$$
\begin{aligned}
\widetilde{P}_{i j}= & \operatorname{GIVPHFWA}_{\lambda}\left(\widetilde{P}_{i j}^{(1)}, \ldots, \widetilde{P}_{i j}^{(l)}\right) \\
= & \left\{\left\langle\sqrt{\left(1-\prod_{k=1}^{l}\left(1-\left(\mu_{\widetilde{P}_{i j}^{(k)}}^{-}\right)^{2 \lambda}\right)^{\xi_{k}}\right)^{1 / \lambda}}, \sqrt{\left(1-\prod_{k=1}^{l}\left(1-\left(\mu_{\widetilde{P}_{i j}^{(k)}}^{+}\right)^{2 \lambda}\right)^{\xi_{k}}\right)^{1 / \lambda}}\right],\right. \\
& {\left[\sqrt{1-\left(1-\prod_{k=1}^{l}\left(1-\left(1-\left(v_{\widetilde{P}_{i j}^{(k)}}^{-}\right)^{2}\right)^{\lambda}\right)^{\xi_{k}}\right)^{1 / \lambda}}, \sqrt{\left.1-\left(1-\prod_{k=1}^{l}\left(1-\left(1-\left(v_{\widetilde{P}_{i j}^{(k)}}^{+}\right)^{2}\right)^{\lambda}\right)^{\lambda}\right)^{\xi_{k}}\right)^{1 / \lambda}}\right], } \\
& \left.\mid\left\langle\widetilde{\mu}_{\widetilde{P}_{i j}}^{(k)}, \tilde{P}_{\widetilde{P}_{i j}}^{(k)}\right\rangle \in \widetilde{P}_{i j}^{(k)}, i=1, \cdots, m, j=1, \cdots, n\right\} .
\end{aligned}
$$

or

$$
\begin{aligned}
& \widetilde{P}_{i j}=\operatorname{GIVPHFWG}_{\lambda}\left(\widetilde{P}_{i j}^{(1)}, \cdots, \widetilde{P}_{i j}^{(l)}\right)
\end{aligned}
$$

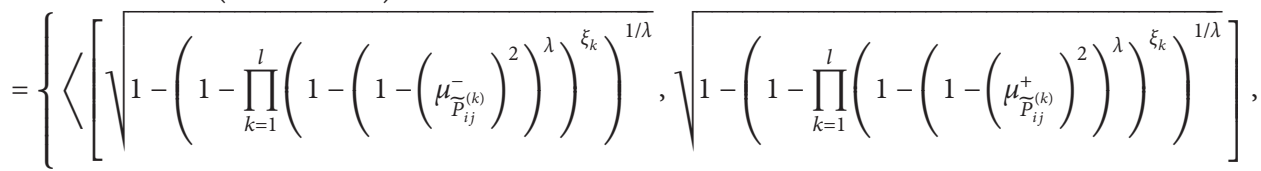

$$
\begin{aligned}
& \left.\left.\left[\sqrt{\left(1-\prod_{k=1}^{l}\left(1-\left(v_{\widetilde{P}_{i j}^{(k)}}^{-}\right)^{2 \lambda}\right)^{\xi_{k}}\right)^{1 / \lambda}}, \sqrt{\left(1-\prod_{k=1}^{l}\left(1-\left(v_{\widetilde{P}_{i j}^{(k)}}^{+}\right)^{2 \lambda}\right)^{\xi_{k}}\right)^{1 / \lambda}}\right]\right\rangle, \mid\left\langle\widetilde{\mu}_{\widetilde{P}_{i j}}^{(k)} \widetilde{\nu}_{\widetilde{P}_{i j}}^{(k)}\right\rangle \in \widetilde{P}_{i j}^{(k)}, i=1, \cdots, m, j=1, \cdots, n\right\} .
\end{aligned}
$$


Step 3: utilize the GIVPHFHA operator (or the GIVPHFHG operator) to aggregate all the preference values $\widetilde{P}_{i}$ again, where $\kappa=\left(\kappa_{1}, \kappa_{2}, \cdots, \kappa_{n}\right)^{T}$ is the associated weight vector. The specific integration operation is as follows:

$$
\begin{aligned}
\widetilde{P}_{i}= & \operatorname{GIVPHFHA}_{\lambda}\left(\widetilde{P}_{i 1}, \cdots, \widetilde{P}_{i n}\right) \\
= & \left\langle\backslash \sqrt{\left(1-\prod_{j=1}^{n}\left(1-\left(\dot{\mu}_{P_{i \sigma(j)}}\right)^{2 \lambda}\right)^{\kappa_{j}}\right)^{1 / \lambda}}, \sqrt{\left(1-\prod_{j=1}^{n}\left(1-\left(\dot{\mu}_{P_{i \sigma(j)}^{+}}\right)^{2 \lambda}\right)^{\kappa_{j}}\right)^{1 / \lambda}}\right], \\
& {\left.\left[\sqrt{1-\left(1-\prod_{j=1}^{n}\left(1-\left(1-\left(\dot{\nu}_{\bar{P}_{i \sigma(j)}}\right)^{2}\right)^{\lambda}\right)^{\kappa_{j}}\right)^{1 / \lambda}}, \sqrt{1-\left(1-\prod_{j=1}^{n}\left(1-\left(1-\left(\dot{\nu}_{P_{i \sigma(j)}^{+}}\right)^{2}\right)^{\lambda}\right)^{\kappa_{j}}\right)^{1 / \lambda}}\right]\right\rangle, } \\
& \mid\left\langle\dot{\tilde{\mu}}_{\left.\left.\widetilde{P}_{i \sigma(j)}, \dot{\tilde{v}}_{P_{i \sigma(j)}}\right\rangle \in \dot{\widetilde{P}}_{i \sigma(j)}, i=1, \cdots, m, j=1, \cdots, n\right\} .}\right.
\end{aligned}
$$

or $\widetilde{P}_{i}=\operatorname{GIVPHFHG}\left(\widetilde{P}_{i 1}, \cdots, \widetilde{P}_{i n}\right)$

$$
\begin{aligned}
& =\left\{\left\langle\sqrt{1-\left(1-\prod_{j=1}^{n}\left(1-\left(1-\left(\ddot{\mu}_{P_{i \sigma(j)}}\right)^{2}\right)^{\lambda}\right)^{\kappa_{j}}\right)^{1 / \lambda}}, \sqrt{1-\left(1-\prod_{j=1}^{n}\left(1-\left(1-\left(\ddot{\mu}_{P_{i \sigma(j)}^{+}}\right)^{2}\right)^{\lambda}\right)^{\kappa_{j}}\right)^{1 / \lambda}}\right],\right. \\
& \left.\left[\sqrt{\left(1-\prod_{j=1}^{n}\left(1-\left(\ddot{\nu}_{P_{i \sigma(j)}}\right)^{2 \lambda}\right)^{\kappa_{j}}\right)^{1 / \lambda}}, \sqrt{\left(1-\prod_{j=1}^{n}\left(1-\left(\ddot{\nu}_{P_{i \sigma(j)}^{+}}\right)^{2 \lambda}\right)^{\kappa_{j}}\right)^{1 / \lambda}}\right]\right\rangle \\
& \left.\mid\left\langle\ddot{\widetilde{v}}_{\widetilde{P}_{i \sigma(j)}}, \ddot{\widetilde{v}}_{P_{i \sigma(j)}}\right\rangle \in \ddot{\widetilde{P}}_{i \sigma(j)}, i=1, \cdots, m, j=1, \cdots, n\right\} .
\end{aligned}
$$

Here, let $\omega=\left(\omega_{1}, \omega_{2}, \cdots, \omega_{n}\right)^{T}$ be the weight vector of the attributes $C_{j}(j=1,2, \cdots, n)$, where $\omega_{j} \in[0,1]$

$$
\begin{aligned}
& \dot{\widetilde{P}}_{i j}=\left(n \times \omega_{j}\right) \otimes \widetilde{P}_{i j} \\
& =\left\{\left\langle\sqrt{1-\left(1-\left(\mu_{P_{i j}}^{\bar{z}}\right)^{2}\right)^{\left(n \times \omega_{j}\right)}}, \sqrt{1-\left(1-\left(\mu_{P_{i j}}^{+}\right)^{2}\right)^{\left(n \times \omega_{j}\right)}}\right],\left[\left(\nu_{\widetilde{P}_{i j}}\right)^{\left(n \times \omega_{j}\right)},\left(\nu_{P_{i j}^{+}}\right)^{\left(n \times \omega_{j}\right)}\right]\right\rangle \\
& \left.\mid\left\langle\widetilde{\mu}_{P_{i j}}, \widetilde{v}_{P_{i j}}\right\rangle \in \widetilde{P}_{i j}, i=1, \cdots, m, j=1, \cdots, n\right\}, \\
& \ddot{\widetilde{P}}_{i j}=\left(\widetilde{P}_{i j}\right)^{\left(n \times \omega_{j}\right)} \\
& =\left\{\left\langle\left[\left(\mu_{P_{i j}}^{-}\right)^{\left(n \times \omega_{j}\right)},\left(\mu_{P_{i j}}^{+}\right)^{\left(n \times \omega_{j}\right)}\right],\left[\sqrt{1-\left(1-\left(\nu_{P_{i j}}^{-}\right)^{2}\right)^{\left(n \times \omega_{j}\right)}}, \sqrt{1-\left(1-\left(v_{P_{i j}^{+}}^{+}\right)^{2}\right)^{\left(n \times \omega_{j}\right)}}\right]\right\rangle\right. \\
& \left.\mid\left\langle\widetilde{\mu}_{P_{i j}}, \widetilde{v}_{P_{i j}}\right\rangle \in \widetilde{P}_{i j}, i=1, \cdots, m, j=1, \cdots, n\right\} .
\end{aligned}
$$


TABLE 1: IVPHFDM $M^{(1)}$.

\begin{tabular}{|c|c|c|c|}
\hline & $C_{1}$ & $C_{2}$ & $C_{3}$ \\
\hline$Y_{1}$ & $\{\langle[0.4,0.5],[0.3,0.5]\rangle\}$ & $\{\langle[0.2,0.3],[0.6,0.8]\rangle\}$ & $\begin{array}{c}\langle\langle[0.6,0.8],[0.1,0.3]\rangle, \\
\langle[0.6,0.7],[0.3,0.3]\rangle, \\
\langle[0.7,0.9],[0.1,0.1]\rangle\}\end{array}$ \\
\hline$Y_{2}$ & $\begin{array}{c}\{\langle[0.1,0.1],[0.8,0.9]\rangle \\
\langle[0.2,0.4],[0.5,0.6]\rangle\}\end{array}$ & $\begin{array}{c}\{\langle[0.4,0.5],[0.5,0.5]\rangle, \\
\langle[0.6,0.7],[0.1,0.2]\rangle, \\
\langle[0.6,0.7],[0.1,0.2]\rangle\}\end{array}$ & $\{\langle[0.8,0.9],[0.1,0.1]\rangle\}$ \\
\hline$Y_{3}$ & $\begin{array}{c}\{\langle[0.2,0.3],[0.6,0.6]\rangle \\
\langle[0.1,0.4],[0.6,0.7]\rangle\}\end{array}$ & $\{\langle[0.8,0.9],[0.1,0.1]\rangle\}$ & $\{\langle[0.3,0.5],[0.4,0.4]\rangle\}$ \\
\hline
\end{tabular}

TABLE 2: IVPHFDM $M^{(2)}$.

\begin{tabular}{|c|c|c|c|}
\hline & $C_{1}$ & $C_{2}$ & $C_{3}$ \\
\hline$Y_{1}$ & $\begin{array}{c}\{\langle[0.7,0.9],[0.1,0.2]\rangle \\
\langle[0.7,0.8],[0.1,0.2]\rangle \\
\langle[0.6,0.8],[0.1,0.1]\rangle\}\end{array}$ & $\begin{array}{c}\{\langle[0.4,0.6],[0.3,0.5]\rangle \\
\langle[0.5,0.7],[0.2,0.3]\rangle\}\end{array}$ & $\{\langle[0.9,0.9],[0.1,0.2]\rangle\}$ \\
\hline$Y_{2}$ & $\{\langle[0.7,0.8],[0.1,0.2]\rangle\}$ & $\{\langle[0.8,0.9],[0.1,0.3]\rangle\}$ & $\begin{array}{c}\{\langle[0.5,0.8],[0.2,0.3]\rangle \\
\langle[0.6,0.8],[0.1,0.2]\rangle\}\end{array}$ \\
\hline$Y_{3}$ & $\{\langle[0.2,0.3],[0.5,0.6]\rangle\}$ & $\begin{array}{c}\{\langle[0.6,0.8],[0.2,0.2]\rangle \\
\langle[0.7,0.9],[0.1,0.1]\rangle\}\end{array}$ & $\begin{array}{c}\langle\langle[0.1,0.3],[0.6,0.7]\rangle \\
\langle[0.2,0.2],[0.7,0.8]\rangle \\
\langle[0.3,0.4],[0.6,0.6]\rangle\}\end{array}$ \\
\hline
\end{tabular}

TABLE 3: IVPHFDM $M^{(3)}$.

\begin{tabular}{|c|c|c|c|}
\hline & $C_{1}$ & $C_{2}$ & $C_{3}$ \\
\hline$Y_{1}$ & $\{\langle[0.3,0.5],[0.4,0.5]\rangle\}$ & $\begin{array}{c}\{\langle[0.2,0.3],[0.5,0.6]\rangle \\
\langle[0.1,0.2],[0.8,0.9]\rangle\}\end{array}$ & $\{\langle[0.7,0.8],[0.2,0.2]\rangle\}$ \\
\hline$Y_{2}$ & $\begin{array}{c}\{\langle[0.4,0.6],[0.3,0.4]\rangle \\
\langle[0.4,0.5],[0.5,0.5]\rangle\}\end{array}$ & $\{\langle[0.1,0.3],[0.6,0.9]\rangle\}$ & $\begin{array}{c}\langle\langle[0.3,0.5],[0.5,0.5]\rangle, \\
\langle[0.1,0.2],[0.7,0.7]\rangle, \\
\langle[0.3,0.4],[0.5,0.6]\rangle\}\end{array}$ \\
\hline$Y_{3}$ & $\begin{array}{c}\{\langle[0.8,0.9],[0.1,0.1]\rangle \\
\langle[0.5,0.7],[0.1,0.2]\rangle\}\end{array}$ & $\begin{array}{c}\{\langle[0.3,0.6],[0.4,0.4]\rangle, \\
\langle[0.4,0.5],[0.4,0.5]\rangle, \\
\langle[0.3,0.5],[0.4,0.5]\rangle\}\end{array}$ & $\{\langle[0.9,0.9],[0.1,0.1]\rangle\}$ \\
\hline
\end{tabular}

Step 4: compute the score values $S\left(\widetilde{P}_{i}\right)$ and the accuracy values $H\left(\widetilde{P}_{i}\right)$ of $\widetilde{P}_{i}(i=1,2, \cdots, m)$ by Definition 15 .

Step 5: get the priority of the alternatives $Y_{i}$ by ranking $S\left(\widetilde{P}_{i}\right)(i=1,2, \cdots, m)$ based on Definition 16, End.

Example 4. A company needs to select a new site for buildings. Three alternatives $Y_{i}(i=1,2,3)$ are available, and the three decision makers $D_{k}(k=1,2,3)$ consider three attributes to decide which project to choose: (1) $C_{1}$ (price), (2) $C_{2}$ (environment), and (3) $C_{3}$ (location). Among the considered attributes, $C_{1}$ is of cost type and $C_{2}$ and $C_{3}$ are of benefit type. The weight vector of the decision makers $D_{k}(k=1,2,3)$ is $\xi=(0.1,0.6,0.3)^{T}$. The weight vector of the attributes $C_{j}(j=1,2,3)$ is $\omega=(0.5,0.2,0.3)^{T}$. Suppose that the decision makers provide their own IVPHFDM $M^{(k)}=\left(\widetilde{\mathscr{P}}_{i j}^{(k)}\right)_{3 \times 3}$ as listed in Tables $1-3$, respectively, where $\widetilde{\mathscr{P}}_{i j}^{(k)}$ is an IVPHFE given by the decision maker $D_{k}$ (Tables 4-7).

Step 1: transform the decision matrix $M^{(k)}$ into the corresponding normalized matrix $N^{(k)}=\left(\widetilde{P}_{i j}^{(k)}\right)_{3 \times 3}$ $(k=1,2,3)$ (in Tables 4-6).
Step 2: suppose $\lambda=5$, and utilize the GIVPHFWA operator to aggregate the three IVPHFDMs $N^{(k)}=\left(\widetilde{P}_{i j}^{(k)}\right)_{3 \times 3}(k=1,2,3) \quad$ into the collective IVPHFDM $N=\left(\widetilde{P}_{i j}\right)_{3 \times 3}$ (in Table 7 ).

Step 3: aggregate all the preference values $\widetilde{P}_{i j}(j=1,2,3)$ in the $i^{\text {th }}$ line of $N$ based on the GIVPHFHA operators, whose associated weighting vector is $\kappa=(0.25,0.65,0.1)^{T}$.

Step 4: compute the three score values as follows:

$$
\begin{aligned}
& S\left(\widetilde{P}_{1}\right)=[0.2736,0.2833], \\
& S\left(\widetilde{P}_{2}\right)=[0.2142,0.2988], \\
& S\left(\widetilde{P}_{3}\right)=[0.2398,0.2741] .
\end{aligned}
$$

Step 5: get the priority of the alternatives by ranking the score functions. We can get the ranking order of all alternatives: $Y_{1}>Y_{3}>Y_{2}$. So, the optimal scheme is $Y_{1}$.

Furthermore, we study the change of the GIVPHFHA and GIVPHFHG operators with the parameter $\lambda$.

In Table 8 , we can find that the score values obtained by the GIVPHFHA operators become bigger as the parameter $\lambda$ 
TABLE 4: IVPHFDM $N^{(1)}$.

\begin{tabular}{cccc}
\hline & $C_{1}$ & $C_{2}$ & $C_{3}$ \\
\hline$Y_{1}$ & $\{\langle[0.3,0.5],[0.4,0.5]\rangle\}$ & $\{\langle[0.2,0.3],[0.6,0.8]\rangle\}$ & $\{\langle[0.6,0.8],[0.1,0.3]\rangle,\langle[0.6,0.7],[0.3,0.3]\rangle$, \\
\hline$Y_{2}$ & $\{\langle[0.8,0.9],[0.1,0.1]\rangle$, & $\{\langle[0.4,0.5],[0.5,0.5]\rangle,\langle[0.6,0.7],[0.1,0.2]\rangle$, & $\{0.9],[0.1,0.1]\rangle\}$ \\
\hline \multirow{2}{*}{$Y_{3}$} & $\langle[0.5,0.6],[0.2,0.4]\rangle\}$ & $\langle[0.6,0.7],[0.1,0.2]\rangle\}$ & $\{\langle[0.8,0.9],[0.1,0.1]\rangle\}$ \\
& $\langle\langle[0.6,0.6],[0.2,0.3]\rangle$, & $\{\langle[0.8,0.9],[0.1,0.1]\rangle\}$ & $\{\langle[0.3,0.5],[0.4,0.4]\rangle\}$ \\
\hline
\end{tabular}

TABLE 5: IVPHFDM $N^{(2)}$.

\begin{tabular}{|c|c|c|}
\hline$C_{1}$ & $\mathrm{C}_{2}$ & $C_{3}$ \\
\hline $\begin{array}{c}Y_{1} \quad\{\langle[0.1,0.2],[0.7,0.9]\rangle,\langle[0.1,0.2],[0.7,0.8]\rangle, \\
\langle[0.1,0.1],[0.6,0.8]\rangle\}\end{array}$ & $\begin{array}{r}\{\langle[0.4,0.6],[0.3,0.5]\rangle \\
\langle[0.5,0.7],[0.2,0.3]\rangle\}\end{array}$ & $\{\langle[0.9,0.9],[0.1,0.2]\rangle\}$ \\
\hline$Y_{2} \quad\{\langle[0.1,0.2],[0.7,0.8]\rangle\}$ & $\{\langle[0.8,0.9],[0.1,0.3]\rangle\}$ & $\{\langle[0.5,0.8],[0.2,0.3]\rangle,\langle[0.6,0.8],[0.1,0.2]\rangle\}$ \\
\hline$\{\langle[0.5,0.6],[0.2,0.3]\rangle\}$ & $\begin{array}{l}\{\langle[0.6,0.8],[0.2,0.2]\rangle \\
\langle[0.7,0.9],[0.1,0.1]\rangle\}\end{array}$ & $\begin{array}{c}\{\langle[0.1,0.3],[0.6,0.7]\rangle,\langle[0.2,0.2],[0.7,0.8]\rangle, \\
\langle[0.3,0.4],[0.6,0.6]\rangle\}\end{array}$ \\
\hline
\end{tabular}

TABLE 6: IVPHFDM $N^{(3)}$.

\begin{tabular}{|c|c|c|c|}
\hline & $C_{1}$ & $\mathrm{C}_{2}$ & $C_{3}$ \\
\hline$Y_{1}$ & $\{\langle[0.4,0.5],[0.3,0.5]\rangle\}$ & $\{\langle[0.2,0.3],[0.5,0.6]\rangle,\langle[0.1,0.2],[0.8,0.9]\rangle\}$ & $\{\langle[0.7,0.8],[0.2,0.2]\rangle\}$ \\
\hline$Y_{2}$ & $\begin{array}{r}\{\langle[0.3,0.4],[0.4,0.6]\rangle \\
\langle[0.5,0.5],[0.4,0.5]\rangle\}\end{array}$ & $\{\langle[0.1,0.3],[0.6,0.9]\rangle\}$ & $\begin{array}{c}\{\langle[0.3,0.5],[0.5,0.5]\rangle,\langle[0.1,0.2],[0.7,0.7]\rangle, \\
\langle[0.3,0.4],[0.5,0.6]\rangle\}\end{array}$ \\
\hline$Y_{3}$ & $\begin{array}{r}\{\langle[0.1,0.1],[0.8,0.9]\rangle \\
\langle[0.1,0.2],[0.5,0.7]\rangle\}\end{array}$ & $\begin{array}{c}\{\langle[0.3,0.6],[0.4,0.4]\rangle,\langle[0.4,0.5],[0.4,0.5]\rangle, \\
\langle[0.3,0.5],[0.4,0.5]\rangle\}\end{array}$ & $\{\langle[0.9,0.9],[0.1,0.1]\rangle\}$ \\
\hline
\end{tabular}

TABLE 7: IVPHFDM $N$.

\begin{tabular}{cccc}
\hline & $C_{1}$ & $C_{2}$ & $C_{3}$ \\
\hline & $\{\langle[0.3553,0.4562],[0.4579,0.6046]\rangle\}$, & $\{\langle[0.3801,0.5702],[0.3631,0.5396]\rangle$, & $\{\langle[0.8648,0.8744],[0.1226,0.2079]\rangle$, \\
$Y_{1} \quad\langle[0.3559,0.4562],[0.4579,0.6014]\rangle$, & $\langle[0.4751,0.6655],[0.2778,0.3778]\rangle$, & $\langle[0.8648,0.8722],[0.1363,0.2079]\rangle$, \\
& $\langle[0.3553,0.4562],[0.4404,0.6014]\rangle$ & $\langle[0.3801,0.5702],[0.3836,0.5614]\rangle$, & $\langle[0.8654,0.8818],[0.1226,0.1862]\rangle\}$, \\
\hline & & $\langle[0.4751,0.6655],[0.2913,0.3870]\rangle\}$ & \\
& & & $\{\langle[0.6419,0.7985],[0.2351,0.3016]\rangle$, \\
$Y_{2}$ & $\langle[0.6387,0.7283],[0.4172,0.4696]\rangle$, & $\{\langle[0.7619,0.8622],[0.1814,0.3798]\rangle$, & $\langle[0.6566,0.7988],[0.1530,0.2351]\rangle$, \\
& $\langle[0.6493,0.4791],[0.4643,0.6207]\rangle$, & $\langle[0.7625,0.8630],[0.1568,0.3451]\rangle$, & $\langle[0.6419,0.7983],[0.2447,0.3150]\rangle$, \\
& $\langle[0.4562,0.4959],[0.4643,0.5790]\rangle\}$ & $\langle[0.7620,0.8630],[0.1568,0.3451]\rangle\}$ & $\langle[0.6566,0.7983],[0.1588,0.2447]\rangle$, \\
& & & $\langle[0.6419,0.7983],[0.2351,0.3104]\rangle$, \\
& & & $\langle[0.6566,0.7983],[0.1530,0.2414]\rangle\}$ \\
\hline & $\langle\langle 0.4767,0.4767],[0.5428,0.6111]\rangle$, & $\langle[0.6998,0.8742],[0.1466,0.1466]\rangle$, & \\
& $\langle[0.4767,0.5567],[0.4758,0.6616]\rangle$, & $\langle[0.6567,0.7985],[0.2247,0.2351]\rangle$, & $\{\langle[0.8094,0.8095],[0.2913,0.2997]\rangle$, \\
& $\langle[0.4767,0.4767],[0.4998,0.6014]\rangle$, & $\langle[0.6998,0.8738],[0.1466,0.1530]\rangle$, & $\langle[0.8094,0.8095],[0.2997,0.3029]\rangle$, \\
& $\langle[0.4767,0.5567],[0.4447,0.6475]\rangle\}$ & $\langle[0.6566,0.7985],[0.2247,0.2351]\rangle$, & $\langle[0.8094,0.8095],[0.2913,0.2913]\rangle\}$ \\
& & $\langle[0.6998,0.8738],[0.1466,0.1530]\rangle\}$ & \\
\hline
\end{tabular}

increases for the same aggregation arguments, and the decision makers can choose the values of $\lambda$ according to their preferences, see that, as the parameter $\lambda$ changes, we have different results. Suppose $\lambda=0.1,0.2, \cdots, 50$, then we analyze the impact of the role of $\lambda$ on the aggregation results, and see the trend in Figure 1. Because of space limitations, we gave the image within the range of $\lambda \in[0.7,11.9]$, the same hereinafter.

From Figure 1, we can see that all of $S\left(\widetilde{P}_{i}\right)(i=1,2,3)$ increase with the increase of $\lambda$. When $\lambda=0.7$, the ranking order of the three alternatives is $Y_{2}>Y_{3}>Y_{1}$ and the best choice is $Y_{2}$; when $\lambda \in[0.8,1.5]$, the ranking order of the three alternatives is $Y_{3}>Y_{2}>Y_{1}$ and the best choice is $Y_{3}$; when $\lambda \in[1.6,11.4]$, the ranking order of the three alternatives is $Y_{1}>Y_{3}>Y_{2}$ and the best choice is $Y_{1}$; when $\lambda \in[11.5,50]$, the ranking order of the three alternatives is $Y_{2}>Y_{1}>Y_{3}$ and the best choice is $Y_{2}$.

In Table 9, we use the GIVPHFHG operators to aggregate the values of the alternatives. We find the score 
TABLE 8: Score values based on the GIVPHFHA operators.

\begin{tabular}{lccc}
\hline & $\lambda=0.1$ & $\lambda=10$ & $\lambda=20$ \\
\hline$Y_{1}$ & {$[-0.4036,-0.3452]$} & {$[0.3207,0.3240]$} & {$[0.3519,0.3530]$} \\
$Y_{2}$ & {$[0.0264,0.0736]$} & {$[0.2865,0.3506]$} & {$[0.3352,0.3988]$} \\
$Y_{3}$ & {$[-0.0184,0.0027]$} & {$[0.2979,0.3073]$} & {$[0.3388,0.3396]$} \\
Ranking & $Y_{2}>Y_{3}>Y_{1}$ & $Y_{1}>Y_{2}>Y_{3}$ & $Y_{2}>Y_{1}>Y_{3}$ \\
\hline & $\lambda=30$ & $\lambda=40$ & $\lambda=50$ \\
$Y_{1}$ & {$[0.3634,0.3641]$} & {$[0.3694,0.3699$} & {$[0.3730,0.3734]$} \\
$Y_{2}$ & {$[0.3533,0.4179]$} & {$[0.3627,0.4279]$} & {$[0.3684,0.4339]$} \\
$Y_{3}$ & {$[0.3543,0.3544]$} & {$[0.3624,0.3624]$} & {$[0.3673,0.3673]$} \\
Ranking & $Y_{2}>Y_{1}>Y_{3}$ & $Y_{2}>Y_{1}>Y_{3}$ & $Y_{2}>Y_{1}>Y_{3}$ \\
\hline
\end{tabular}

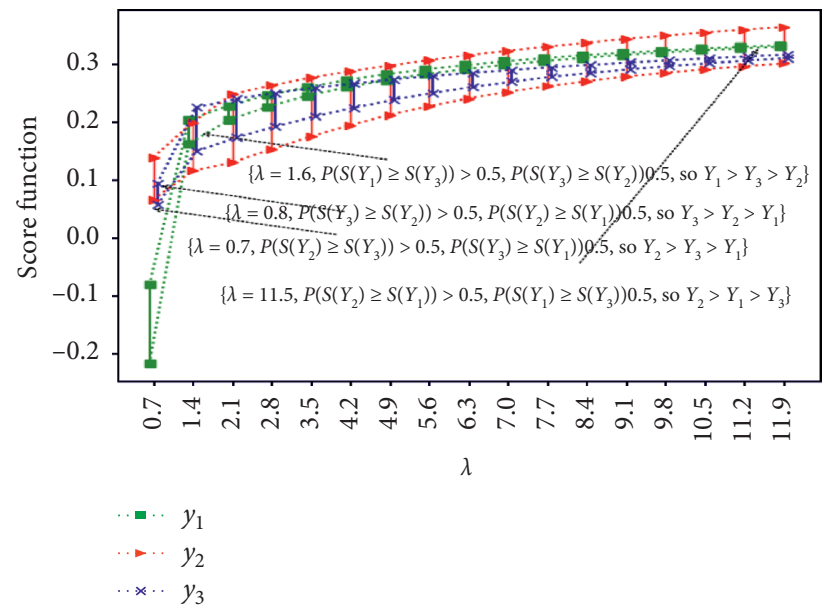

FIgURE 1: Variation of the score functions of the GIVPHFHA operators by the parameter $\lambda$.

TABLE 9: Score values based on the GIVPHFHG operators.

\begin{tabular}{lccc}
\hline & $\lambda=0.1$ & $\lambda=10$ & $\lambda=20$ \\
\hline$Y_{1}$ & {$[0.392,0.4493]$} & {$[-0.3225,-0.2264]$} & {$[-0.3633,-0.2567]$} \\
$Y_{2}$ & {$[-0.0184,0.0047]$} & {$[-0.2448,-0.1454]$} & {$[-0.2774,-0.1621]$} \\
$Y_{3}$ & {$[-0.0148,0.0178]$} & {$[-0.3424,-0.2848]$} & {$[-0.3950,-0.3340]$} \\
Ranking & $Y_{1}>Y_{2}>Y_{3}$ & $Y_{2}>Y_{1}>Y_{3}$ & $Y_{2}>Y_{1}>Y_{3}$ \\
\hline & $\lambda=30$ & $\lambda=40$ & $\lambda=50$ \\
$Y_{1}$ & {$[-0.3793,-0.2681]$} & {$[-0.3877,-0.2344]$} & {$[-0.3928,-0.2011]$} \\
$Y_{2}$ & {$[-0.2899,-0.0968]$} & {$[-0.2217,-0.0990]$} & {$[-0.2244,-0.1006]$} \\
$Y_{3}$ & {$[-0.4149,-0.3524]$} & {$[-0.4254,-0.3619]$} & {$[-0.4318,-0.3677]$} \\
Ranking & $Y_{2}>Y_{1}>Y_{3}$ & $Y_{2}>Y_{1}>Y_{3}$ & $Y_{2}>Y_{1}>Y_{3}$ \\
\hline
\end{tabular}

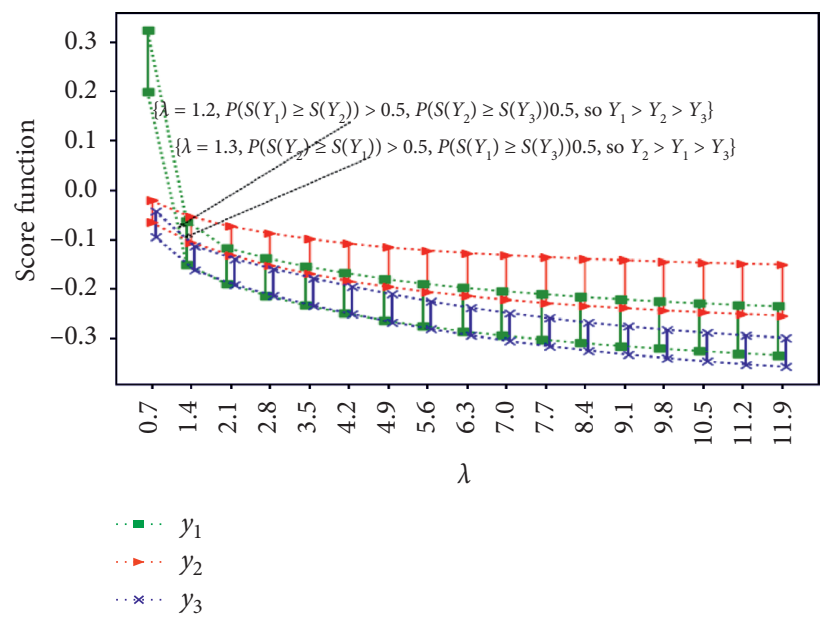

Figure 2: Variation of the score functions of the GIVPHFHG operators by the parameter $\lambda$. 
values obtained by the GIVPHFHG operators become smaller as the parameter $\lambda$ increases for the same aggregation arguments, and the decision makers can choose the values of $\lambda$ according to their preferences. See the details in Figure 2.

From Figure 2, we can see that all of $S\left(\widetilde{P}_{i}\right)(i=1,2,3)$ decrease with the increase of $\lambda$. When $\lambda \in[0.7,1.2]$, the ranking order of the three alternatives is $Y_{1}>Y_{2}>Y_{3}$ and the best choice is $Y_{1}$; when $\lambda \in[1.3,50]$, the ranking order of the three alternatives is $Y_{2}>Y_{1}>Y_{3}$ and the best choice is $Y_{2}$.

\section{Conclusions}

This paper extends IVHFs and IVPFs to IVPHFSs. Firstly, the new score functions and accuracy functions of IVPHFSs are introduced to compare the size of IVPHFEs based on the comparison of interval numbers. Then, we focus on a new series of interval-valued Pythagorean hesitant fuzzy aggregation operators, including the IVPHFWA, IVPHFWG, GIVPHFWA, GIVPHFWG, IVPHFOWA, IVPHFOWG, GIVPHFOWA, GIVPHFOWG, IVPHFHA, IVPHFHG, GIVPHFHA, and GIVPHFHG operators. The relations of these operators are developed. Furthermore, a new approach has been developed based on the proposed operators to solve the MAGDM problems under the IVPHF environment. Finally, a numerical example is used to illustrate the effectiveness and feasibility of our proposed method.

The following work is to enhance the study of the aggregation operators of weighted IVPHFSs; we shall develop the new potential application of the proposed operators in another field such as clustering analysis, image processing, pattern recognition, and so on. We hope that these will enrich and provide more new ideas and new methods for these fields under the interval-valued Pythagorean hesitant fuzzy environment.

\section{Abbreviations}

GIVPHFHA: Generalized interval-valued Pythagorean hesitant fuzzy hybrid averaging

GIVPHFHG: Generalized interval-valued Pythagorean hesitant fuzzy hybrid geometric

GIVPHFOWA: Generalized interval-valued Pythagorean hesitant fuzzy ordered weighted averaging

GIVPHFOWG: Generalized interval-valued Pythagorean hesitant fuzzy ordered weighted geometric

GIVPHFWA: Generalized interval-valued Pythagorean hesitant fuzzy weighted averaging

GIVPHFWG: Generalized interval-valued Pythagorean hesitant fuzzy weighted geometric

HFE:

HFS:

IVHFS:

IVHFE:

IVPHFDM:

IVPHFE:
IVPHFHA: Interval-valued Pythagorean hesitant fuzzy

IVPHFHG: $\quad$ hybrid averaging hybrid geometric

IVPHFOWA: Interval-valued Pythagorean hesitant fuzzy ordered weighted averaging

IVPHFOWG: Interval-valued Pythagorean hesitant fuzzy ordered weighted geometric

IVPHFWA: Interval-valued Pythagorean hesitant fuzzy weighted averaging

IVPHFWG: Interval-valued Pythagorean hesitant fuzzy weighted geometric

IVPHFS: Interval-valued Pythagorean hesitant fuzzy set

IVPFE: Interval-valued Pythagorean fuzzy element

IVPFS: Interval-valued Pythagorean fuzzy set

MAGDM: $\quad$ Multiattribute group decision-making

PHFE: $\quad$ Pythagorean hesitant fuzzy element

PHFS: Pythagorean hesitant fuzzy set.

\section{Data Availability}

The data used to support the findings of this study are available from the corresponding author upon request.

\section{Conflicts of Interest}

The authors declare that they have no conflicts of interest.

\section{Acknowledgments}

The work was supported by the National Natural Science Foundation of China (nos. 61806001, 71771001, 71701001, and 71871001), Natural Science Foundation of Anhui Province (no. 1708085MF163), Natural Science Foundation for Distinguished Young Scholars of Anhui Province (no. 1908085J03), Research Funding Project of Academic and technical leaders and reserve candidates in Anhui Province (no. 2018H179), and Provincial Natural Science Research Project of Anhui Colleges (no. KJ2017A026).

\section{References}

[1] L. A. Zadeh, "Fuzzy sets," Information and Control, vol. 8, no. 3, pp. 338-353, 1965.

[2] K. T. Atanassov, "Intuitionistic fuzzy sets," Fuzzy Sets and Systems, vol. 20, no. 1, pp. 87-96, 1986.

[3] K. Atanassov and G. Gargov, "Interval valued intuitionistic fuzzy sets," Fuzzy Sets and Systems, vol. 31, no. 3, pp. 343-349, 1989.

[4] L. A. Zadeh, "The concept of a linguistic variable and its application to approximate reasoning-I," Information Sciences, vol. 8, no. 3, pp. 199-249, 1975.

[5] J. M. Mendel, R. I. John, and F. Liu, "Interval type-2 fuzzy logic systems made simple," IEEE Transactions on Fuzzy Systems, vol. 14, no. 6, pp. 808-821, 2006.

[6] R. R. Yager, "Generalized orthopair fuzzy sets," IEEE Transactions on Fuzzy Systems, vol. 26, no. 5, pp. 1222-1230, 2017. 
[7] W. Pedrycz, "Fuzzy sets in pattern recognition: methodology and methods," Pattern Recognition, vol. 23, no. 1-2, pp. 121-146, 1990.

[8] J. Ye, "Cosine similarity measures for intuitionistic fuzzy sets and their applications," Mathematical and Computer Modelling, vol. 53, no. 1-2, pp. 91-97, 2011.

[9] F. Liu, "An efficient centroid type-reduction strategy for general type-2 fuzzy logic system," Information Sciences, vol. 178, no. 9, pp. 2224-2236, 2008.

[10] P. Liu and P. Wang, "Some q-Rung orthopair fuzzy aggregation operators and their applications to multiple-attribute decision making," International Journal of Intelligent Systems, vol. 33, no. 2, pp. 259-280, 2018.

[11] P. Liu and J. Liu, "Some q-rung orthopai fuzzy Bonferroni mean operators and their application to multi-attribute group decision making," International Journal of Intelligent Systems, vol. 33, no. 2, pp. 315-347, 2018.

[12] P. Liu and P. Wang, "Multiple-attribute decision-making based on archimedean Bonferroni operators of q-Rung orthopair fuzzy numbers," IEEE Transactions on Fuzzy Systems, vol. 27, no. 5, pp. 834-848, 2019.

[13] P. Liu, S.-M. Chen, and P. Wang, "Multiple-attribute group decision-making based on q-rung orthopair fuzzy power Maclaurin symmetric mean operators," IEEE Transactions on Systems, Man, and Cybernetics: Systems, pp. 1-16, 2019.

[14] T. Wu, X. Liu, and F. Liu, "An interval type-2 fuzzy TOPSIS model for large scale group decision making problems with social network information," Information Sciences, vol. 432, pp. 392-410, 2018.

[15] T. Wu, X. Liu, and J. Qin, "A linguistic solution for double large-scale group decision-making in E-commerce," Computers \& Industrial Engineering, vol. 116, pp. 97-112, 2018.

[16] Q. Wu, P. Wu, L. Zhou, H. Chen, and X. Guan, "Some new Hamacher aggregation operators under single-valued neutrosophic 2-tuple linguistic environment and their applications to multi-attribute group decision making," Computers \& Industrial Engineering, vol. 116, pp. 144-162, 2018.

[17] Q. Wu, W. Lin, L. Zhou, Y. Chen, and H. Chen, "Enhancing multiple attribute group decision making flexibility based on information fusion technique and hesitant Pythagorean fuzzy sets," Computers \& Industrial Engineering, vol. 127, pp. 954-970, 2019.

[18] Q. Wu, L. Zhou, Y. Chen, and H. Chen, "An integrated approach to green supplier selection based on the interval type-2 fuzzy best-worst and extended VIKOR methods," Information Sciences, vol. 502, pp. 394-417, 2019.

[19] V. Torra and Y. Narukawa, "On hesitant fuzzy sets and decision," in Proceedings of the 18th IEEE International Conference on Fuzzy Systems, pp. 1378-1382, Jeju Island, Korea, 2009.

[20] V. Torra, "Hesitant fuzzy sets," International Journal of Intelligent Systems, vol. 25, no. 6, pp. 529-539, 2010.

[21] R. M. Rodríguez, L. Martínez, V. Torra, Z. S. Xu, and F. Herrera, "Hesitant fuzzy sets: state of the art and future directions," International Journal of Intelligent Systems, vol. 29, no. 6, pp. 495-524, 2014.

[22] B. Zhu, Z. Xu, and M. Xia, "Dual hesitant fuzzy sets," Journal of Applied Mathematics, vol. 2012, Article ID 879629, 13 pages, 2012.

[23] Y. Ju, X. liu, and S. Yang, "Interval-valued dual hesitant fuzzy aggregation operators and their applications to multiple attribute decision making," Journal of Intelligent \& Fuzzy Systems, vol. 27, no. 3, pp. 1203-1218, 2014.

[24] Y. Zang, X. Zhao, and S. Li, "Interval-valued dual hesitant fuzzy heronian mean aggregation operators and their application to multi-attribute decision making," International Journal of Computational Intelligence and Applications, vol. 17, no. 1, Article ID 1850005, 2018.

[25] J.-j. Peng, J.-q. Wang, X.-h. Wu, H.-y. Zhang, and X.-h. Chen, "The fuzzy cross-entropy for intuitionistic hesitant fuzzy sets and their application in multi-criteria decision-making," International Journal of Systems Science, vol. 46, no. 13, pp. 2335-2350, 2015.

[26] N. Chen, Z. Xu, and M. Xia, "Interval-valued hesitant preference relations and their applications to group decision making," Knowledge-Based Systems, vol. 37, pp. 528-540, 2013.

[27] N. Chen and Z. Xu, "Properties of interval-valued hesitant fuzzy sets," Journal of Intelligent \& Fuzzy Systems, vol. 27, no. 1, pp. 143-158, 2014.

[28] W. Zeng, D. Li, and Q. Yin, "Weighted interval-valued hesitant fuzzy sets and its application in group decision making," International Journal of Fuzzy Systems, vol. 12, pp. 1-12, 2019.

[29] Z. Zhang, "Interval-valued intuitionistic hesitant fuzzy aggregation operators and their application in group decisionmaking," Journal of Applied Mathematics, vol. 2013, pp. 1-33, 2013.

[30] M. Xia and Z. Xu, "Hesitant fuzzy information aggregation in decision making," International Journal of Approximate Reasoning, vol. 52, no. 3, pp. 395-407, 2011.

[31] Z. Zhang, "Hesitant fuzzy power aggregation operators and their application to multiple attribute group decision making," Information Sciences, vol. 234, pp. 150-181, 2013.

[32] X. Zhang and Z. Xu, "Hesitant fuzzy agglomerative hierarchical clustering algorithms," International Journal of Systems Science, vol. 46, no. 3, pp. 562-576, 2015.

[33] R. R. Yager, "Pythagorean fuzzy subsets," in Proceeding of the Joint IFSA World Congress and NAFIPS Annual Meeting, pp. 57-61, Edmonton, Canada, 2013.

[34] R. R. Yager and A. M. Abbasov, "Pythagorean membership grades, complex numbers, and decision making," International Journal of Intelligent Systems, vol. 28, no. 5, pp. 436452, 2013.

[35] R. R. Yager, "Pythagorean membership grades in multicriteria decision making," IEEE Transactions on Fuzzy Systems, vol. 22, no. 4, pp. 958-965, 2014.

[36] R. R. Yager, "Properties and applications of Pythagorean fuzzy sets," Imprecision and Uncertainty in Information Representation and Processing. Studies in Fuzziness and Soft Computing, Springer, Cham, Switzerland, 2016.

[37] X. Zhang and Z. S. Xu, "Extension of TOPSIS to multiple criteria decision making with Pythagorean fuzzy sets," International Journal of Intelligent Systems, vol. 29, no. 12, pp. 1061-1078, 2015.

[38] P. Ren, Z. Xu, and X. Gou, "Pythagorean fuzzy TODIM approach to multi-criteria decision making," Applied Soft Computing, vol. 42, pp. 246-259, 2016.

[39] Z. Liu, P. Liu, W. Liu, and J. Pang, "Pythagorean uncertain linguistic partitioned Bonferroni mean operators and their application in multi-attribute decision making," Journal of Intelligent \& Fuzzy Systems, vol. 32, no. 3, pp. 2779-2790, 2017.

[40] F. Teng, Z. Liu, and P. Liu, "Some power Maclaurin symmetric mean aggregation operators based on Pythagorean fuzzy linguistic numbers and their application to group decision making," International Journal of Intelligent Systems, vol. 33, no. 9, pp. 1949-1985, 2018. 
[41] X. Peng and Y. Yang, "Fundamental properties of intervalvalued Pythagorean fuzzy aggregation operators," International Journal of Intelligent Systems, vol. 31, no. 5, pp. 444-487, 2016.

[42] K. Rahman and S. Abdullah, "Generalized interval-valued Pythagorean fuzzy aggregation operators and their application to group decision-making," Granular Computing, vol. 1, pp. 1-11, 2018.

[43] K. Rahman, S. Abdullah, and A. Ali, "Some induced aggregation operators based on interval-valued Pythagorean fuzzy numbers," Granular Computing, vol. 1, pp. 1-10, 2018.

[44] L. Yi, Y. Qin, and H. Yun, "Multiple criteria decision making with probabilities in interval-valued Pythagorean fuzzy setting," International Journal of Fuzzy Systems, vol. 20, no. 2, pp. 558-571, 2017.

[45] D. C. Liang, A. P. Darko, and Z. S. Xu, "Interval-valued Pythagorean fuzzy extended Bonferroni mean for dealing with heterogenous relationship among attributes," International Journal of Intelligent Systems, pp. 1-31, 2018.

[46] W. F. Liu and X. He, "Pythagorean hesitant fuzzy sets," Fuzzy Systems and Mathematics, vol. 30, no. 4, pp. 107-115, 2016, in Chinese.

[47] M. S. A. Khan, S. Abdullah, A. Ali, N. Siddiqui, and F. Amin, "Pythagorean hesitant fuzzy sets and their application to group decision making with incomplete weight information," Journal of Intelligent \& Fuzzy Systems, vol. 33, no. 6, pp. 3971-3985, 2017.

[48] G. Wei, M. Lu, X. Tang, and Y. Wei, "Pythagorean hesitant fuzzy Hamacher aggregation operators and their application to multiple attribute decision making," International Journal of Intelligent Systems, vol. 33, no. 6, pp. 1197-1233, 2018.

[49] H. Dawood, Theories of Interval Arithmetic: Mathematical Foundations and Applications, LAP Lambert Academic Publishing, Saarbrücken, Germany, 2011.

[50] Z. S. Xu and Q. L. Da, "The uncertain OWA operator," International Journal of Intelligent Systems, vol. 17, no. 6, pp. 569-575, 2002.

[51] H. Garg, "New exponential operational laws and their aggregation operators for interval-valued Pythagorean fuzzy multicriteria decision-making," International Journal of Intelligent Systems, vol. 33, no. 3, pp. 653-683, 2018.

[52] Z. Xu and R. R. Yager, "Some geometric aggregation operators based on intuitionistic fuzzy sets," International Journal of General Systems, vol. 35, no. 4, pp. 417-433, 2006.

[53] V. Torra and Y. Narukawa, Modeling Decisions: Information Fusion and Aggregation Operators, Springer, Cham, Switzerland, 2007.

[54] Z. S. Xu and X. Cai, "Nonlinear optimization models for multiple attribute group decision making with intuitionistic fuzzy information," International Journal of Intelligent Systems, vol. 25, no. 6, pp. 489-513, 2010. 